\title{
IMPLICIT AND EXPLICIT MEMORY IN DEPRESSION/ DYSPHORIA: AN ANALYSIS OF THE CONTRIBUTION OF PERCEPTUAL AND CONCEPTUAL PROCESSES
}

\author{
A thesis presented to the Victoria University \\ of Wellington in fulfilment of the requirements \\ for the degree of Doctor of Philosophy
}

\author{
By
}

William Jenkins

September, 1998 


\section{Contents}

Page

List of tables 1

List of figures $\quad 2$

$\begin{array}{ll}\text { Acknowledgements } & 3\end{array}$

$\begin{array}{ll}\text { Abstract } & 4\end{array}$

1.0 Introduction 6

1.1 The processing model $\quad 15$

1.2 The processing versus memory systems models: the contribution of Blaxton $\quad 18$

1.3 Problems with the processing model 21

1.4 The processing versus memory systems models:

1.5 Implicit - explicit memory research with clinical participants 25

1.6 Depression and memory research (general) 28

1.7 Depression and implicit - explicit memory research 30

1.8 Models used to explain dissociations 39

1.9 The processing model and depression - implicit memory research $\quad 44$

2.0 Experiment one 47

2.1 Method 50

2.2 Results 59

2.3 Discussion 65

3.0 Experiment two 69

3.1 Method 72

$\begin{array}{lll}3.2 & \text { Results } & 78\end{array}$

3.3 Discussion 82

4.0 Experiment three 88

4.1 Method 89

4.2 Results 96

$\begin{array}{lll}4.3 & \text { Discussion } & 101\end{array}$ 
$\begin{array}{lll}5.0 & \text { Experiment four } & 107\end{array}$

5.1 Method 110

$\begin{array}{lll}5.2 & \text { Results } & 116\end{array}$

5.3 Discussion 122

6.0 General discussion 126

6.1 The effects of depression on implicit - explicit tests of memory 128

$\begin{array}{lll}6.2 & \text { The processing model } & 129\end{array}$

6.3 The memory systems model 132

6.4 Time for a combined model? 133

6.5 The utility of the revised memory systems model 135

6.6 The integration activation model 137

6.7 Resource allocation versus cognitive initiative model 139

$\begin{array}{lll}6.8 & \text { Problems } & 141\end{array}$

6.9 Future research 143

6.10 Other clinical groups 144

6.11 The use of student participants as analogues for $\begin{array}{ll}\text { clinical depression } & 147\end{array}$

6.12 Closing summary 152

$\begin{array}{lll}7.0 & \text { References } & 155\end{array}$

$\begin{array}{lll}8.0 & \text { Appendix } & 163\end{array}$ 
1.1 Summary of previous depression / dysphoria implicit - explicit memory research

2.1 Mean correct generate completions for the 3 blocks and 2 lists for experimental and control participants (Experiment 1)

2.2 Mean encoding condition completions: graphemic cued recall 60

2.3 Mean encoding condition completions: word fragment completion

2.4 Mean encoding condition completions: semantic cued recall

2.5 Mean encoding condition completions: category association

3.1 Mean correct generate completions for the 3 blocks and 2 lists for experimental and control participants (Experiment 2)

3.2 Mean encoding condition completions: perceptual identification

3.3 Mean encoding condition completions: anagrams 80

3.4 Mean encoding condition completions: free recall 81

4.1 Mean correct generate completions for the 3 blocks and 2 lists for experimental and control participants (Experiment 3)

4.2 Mean encoding condition completions: word fragment completion

4.3 Mean encoding condition completions: category association 98

4.4 Mean encoding condition completions: free recall 99

5.1 Mean correct generate completions for the 4 blocks and 2 lists for experimental and control participants (Experiment 4)

5.2 Mean encoding condition completions: implicit word association

5.3 Mean encoding condition completions: explicit word association

5.4 Combined test encoding means by encoding type for experimental and control participants

5.5 Implicit and explicit encoding condition means 122

6.1 Overview of Experiments 1 - $4 \quad 127$ 
List of figures

3.1 Mean correct completions: explicit free recall (Experiment 2)

4.1 Mean correct completions: implicit category association (Experiment 3)

4.2 Mean correct completions: explicit free recall (Experiment 3)

5.1 Mean correct completions: implicit word association (Experiment 4)

5.2 Mean correct completions: explicit word association (Experiment 4) 


\section{Acknowledgements}

The author would like to thank the many people who have provided support, friendship and advice during the course of this work.

Firstly to my supervisor Dr John McDowall for his support, good humour and advice. I would also like to thank those people who really make the psychology department the friendly and efficient place it is to work in. The secretaries (Ngaire Lavery and Jaana Montgomery), the technicians (Keith Riach and Doug Drysdale) and the computer programmers (Linton Miller, David Crossan and Douglas Flux).

Thanks also for the clinical psychologists and psychiatrists who passed on my consent forms and arranged for clients to participate in Experiment 3.

Thanks also to my many good friends who must have been wondering where I have been these last few years. Last and not least thanks to my parents for their help and support. In particular a big thanks to my mother, Margaret Ellen Jenkins (1932 - 1998) whose example of courage and patience helped make this thesis possible. 
Earlier studies have shown impaired explicit test and normal implicit test performance in participants classified as depressed. A number of different models have been put forward to explain this 'typical' test dissociation including the memory systems, processing, and activation - elaboration models. Blaxton $(1989,1992)$ has pointed out that to date most test designs have confounded the memory systems and processing models. The aim of this series of experiments was to systematically compare the effects of depression on the processing and memory systems models and in so doing provide a more precise explanation for the effects of depression on human memory.

Across Experiments 1 - 4 the performance of participants with depression or dysphoria were examined on implicit and explicit memory tests which were designed to tap either predominantly perceptual or conceptual processes. In Experiment 1 the conceptual tests of category association (implicit) and semantic cued recall (explicit) were compared with the perceptual tests of word fragment completion (implicit) and graphemic cued recall (explicit). In Experiment 2 the perceptual tests of perceptual identification (implicit) and the 'mixed' test of anagram solution (implicit) were compared with the conceptual free recall test (explicit). Both experiments used dysphoric university students and found no effects of dysphoria in comparison to normal controls matched for age, sex and education levels.

Experiment 3 compared the conceptual category association (implicit) and free recall (explicit) tests with the perceptual word fragment completion test (implicit) using participants diagnosed with major depression disorder. This revealed significant impairments in both the conceptual tests while the perceptual test was intact. Experiment 4 compared the implicit word association test with the explicit word association test using dysphoric university students. Experiment 4 found that dysphoric participants were impaired in performing the explicit test while the implicit test remained intact. 
These findings suggest that dysphoria has no effect on implicit tests, but can effect conceptual explicit test measures. Clinical depression effects both conceptual implicit and conceptual explicit test measures. While these results support aspects of both the memory systems and processing models these findings may be best accommodated by a model which combines these models. The revised memory systems model is discussed as one means of achieving this. 


\subsection{Introduction.}

Over the last 10 years a quiet revolution has occurred in human memory research. Up until the late 1980's the vast majority of memory research with normal or memory impaired participants relied on tests which instructed participants to deliberately or consciously recall items from an earlier stage in an experiment. Tests of this nature have been described as explicit memory tests. Schacter (1987) has described this type of test as "requiring conscious recollection of recently presented information" (p. 501).

However Graf and Masson (1993) have pointed out that a typical explicit memory test taps only a small amount or the 'tip of the iceberg' of how memory for recent events can influence daily life. During the last several years cognitive psychologists have paid extensive attention to experimental situations in which information that was encoded during a particular episode is subsequently expressed without conscious or deliberate recollection. The memory transfer tapped by this form of experiment has been described as implicit memory (Roediger, 1990). Implicit memory has been defined as "memory for information that was acquired during a specific episode and is expressed on tests for which participants are not required and are frequently unable, to deliberately or consciously recollect the previously studied information". (Schacter, 1990, p. 338).

In many ways the techniques for studying implicit memory is similar to the laboratory techniques used to study explicit memory. Although laboratory experiments which make use of implicit and explicit tests can vary widely in their design there are a number of common elements which nearly all experiments would use.

In both cases participants are first exposed to material, usually a list of words or pictures during a study phase (this is described as the encoding phase). In a later test phase participants in an explicit memory task are asked to recall these words or pictures or recognise them among a range of plausible 
alternatives. In either case the participants are asked to consciously retrieve the items presented during the study phase.

In an implicit memory task participants are not directed to intentionally recall the items that were presented earlier, instead they are given some 'unrelated' task such as identifying fragmented forms of the pictures or words. Typically there is an increased tendency to complete fragments using words that were presented earlier (Roediger \& McDermott, 1993). This facilitation of performance is called priming and this is taken as an index of implicit memory transfer from the study to the test phases.

\section{Some common implicit - explicit tests}

The list of implicit memory tests in use is increasing all the time. By its broadest definition implicit memory tests can include any judgement or test that is (a) affected by past experience and (b) given under instructions or conditions when participants are not explicitly required to remember earlier events (Roediger \& McDermott, 1993). While the tests outlined below all depend on some form of verbal/word priming (this is the most common form of measure that is used) other stimuli have also been used in implicit memory research. These have included the priming of pictures, objects, motor learning and conditioning tests.

As noted previously priming or implicit memory experiments involve at least two phases. In the first phase participants are asked to study a list of material, for example a list of words. Quite often participants are asked to perform some form of orienting task as they study this material. For instance the participant may be asked to imagine a scene involving the studied word. As an example if the studied word was elephant the participant may imagine a view of a elephant standing in a plain. Following this first phase participants may be given various filler tasks (e.g. simple problems to solve) before being given the memory test. In most implicit memory tests participants are given some form of representation (this is often described as a cue) of the word studied during the 
encoding phase, for example a word fragment (c_mp_t_r). Again interest is centred on priming, or the difference in speed or accuracy with which participants perform the task after relevant study experience, compared to when no prior experiences have occurred.

A common implicit memory test is the perceptual (word) identification test (e.g. Jacoby \& Dallas, 1981). This test involves briefly flashing a word usually beginning at 35 millisecond $(\mathrm{msec}$ ) presentation. The participant is asked to correctly identify the word and if they cannot the presentation time is increased on each subsequent presentation until the correct identification is made. Typically prior exposure to a relevant word during study results in faster identification times.

The word stem completion test (e.g. Graf, Squire \& Mandler, 1984) involves presenting the participant with a three or four letter word stem, for example ele___ (for elephant) and requesting the participant to complete the stem with the first word that comes to mind. The word stem completion test is similar to the word fragment completion test (e.g. Roediger, Weldon, Stadler \& Riegler, 1992). Here a word fragment is presented to the participant, for example e_e_h__t and the participant is asked to complete the fragment with the first real word that comes to mind. In each case prior exposure to a word will result in an increased tendency to complete a stem or fragment with a previously studied word.

The anagram solution test (e.g. Srinivas \& Roediger, 1990) involves presenting the participant with an anagram, that is a word which has had its letters rearranged into a 'random' order (e.g. bleta for table). The participant is asked to unscramble the letters to form a real word. Prior exposure to a relevant word typically results in an increased likelihood that the anagram will be solved with the study word.

The lexical decision test (e.g. Duchek \& Heely, 1989) involves presenting the participant with a word or non-word (a string of letters e.g. raddle that is often pronounceable but does not spell an English word). The participant is required to decide whether the string is a word or non-word. Prior exposure to 
the relevant word during encoding usually results in a faster decision latency for that word compared to other real unrelated words or non-words.

The word association test (e.g. Shimamura \& Squire, 1984) provides the participant with a stimulus word, for instance tusk. The participant is then asked to write down the first associated word that comes to mind, for example rhino. Each of the word cues presented at test will have at least one word that it is closely associated with (usually in meaning) presented during encoding. Prior exposure to this study word should result in an increased likelihood that the participant will associate this word with the word cue used at test.

A similar test is the category instance association test (e.g. Srinivas \& Squire, 1984). Here participants are provided with a category such as animals. Participants are usually given a time limit to write down as many examples of that category that they can think of, for example elephants. During the encoding phase participants are presented with a number of examples that belong to a particular category. Again prior exposure to these relevant words should result in an increased likelihood that these words will be associated with a particular category.

\section{Test dissociations}

The distinction between explicit and implicit tests is especially important in that typically these tests can be dissociated from each other (for a review see Richardson-Klavehn \& Bjork, 1988; Schacter, 1987; Tulving \& Schacter, 1990). For example it has been commonly observed that the manipulation of independent variables such as modality (e.g. requiring participants to encode study items by visual means and then separately by auditory means) can have a large effect on test $Y$, for instance the explicit test while the opposite or no effect is observed in test $\mathrm{X}$, the implicit test.

Similarly Jacoby and Dallas (1981) manipulated the depth of processing during encoding by asking participants to provide either semantic (meaning based) or structural judgements about a series of familiar words. Subsequent 
memory was tested using an explicit yes/no recognition test and an implicit perceptual identification test. Recognition was higher after semantic judgements, than after structural judgements whereas similar levels of priming were obtained from each encoding manipulation for the implicit perceptual identification test. Schacter (1987) notes that dissociations between implicit and explicit tests have been obtained across a wide range of test domains including savings measured during relearning, subliminal experiments as well as verbal priming experiments.

Dissociations have also been found by comparing test performance between neuropsychological or 'special' populations. For instance those in various stages of intoxication (Hashtroudi, Parker, De Lisi, Wyatt \& Mutter, 1984), the aged (Howard, 1991) and children (Naito, 1990) have shown a pattern of impaired explicit but relatively intact implicit test performance.

An important reason why cognitive psychologists initially became interested in implicit memory tests was the observation that even in densely amnesic patients, performance on implicit tests seemed resistant to the dramatic forgetting observed in explicit tests (Warrington \& Weiskrantz, 1968,1970). Warrington and Weiskrantz (1970), compared the performance of a group of amnesic participants with a control group and found intact performance in a number of implicit tests (word fragment completion, word stem completion) in both groups while the amnesic group exhibited significant deficits for the explicit tests (free recall and recognition).

Taken together, findings such as these have led to the conclusion that implicit tests of retention measure a form of 'knowledge' that in some ways differs from that measured by explicit tests of memory (Roediger \& McDermott, 1993). Roediger (1990) notes that dissociations obtained from amnesic populations are especially powerful in that amnesic participants are usually unaware that they know the material when they are tested directly or explicitly, yet perform within a normal range on implicit tests. This finding has led many researchers to suggest that the dissociation between implicit and explicit memory tests can be explained with reference to different cognitive processes 
and/ or systems and that these are tapped by these different tests (see Watkins, Mathews, Williamson \& Fuller, 1992 for a discussion on this point).

\section{Test terminology}

It should be noted that Roediger (1990) has pointed out that the terminology used in the literature is often confused or poorly defined. For instance Schacter (1987) has referred to the terms implicit and explicit memory as a means of referring to two different forms of memory as well as two different classes of test. There is no direct map necessarily between a type of test and a form of memory experience, let alone a hypothesised memory system or process (Schacter, 1990). Schacter, Bowers and Booker (1989) noted that when Graf and Schacter (1985) introduced the concepts of implicit and explicit memory they tried to provide only a description of what the test required the participants to do rather than a description of the processes or memory systems involved and as a result, avoid any particular theoretical position.

Roediger (1990) notes that some prefer the use of the terms direct and indirect memory (Richardson-Klavehn \& Bjork, 1988) however this term is limited in usefulness to describing a type of test rather than a 'mode of memory retrieval'. Schacter (1990) has also pointed out that these terms can also suffer the same problems as implicit and explicit memory, in that it is possible that participants can realise the nature of the test and thus turn an indirect test into a direct test. Roediger (1990) proposes that alternative terms could be 'retention with awareness' and 'retention without awareness' although he notes that it can be difficult to control and provide a check for the presence of awareness in implicit tests. Similarly Jacoby (1991) has referred to explicit tests as those displaying controlled uses of memory and implicit tests (in the pure case) as accessing automatic uses of memory. However unless one is able to independently measure and control these processes such a description is often limited in direct usefulness. 
In the present study the terms explicit and implicit memory tests will refer only to types of tasks and not to different forms of memory or 'psychological states'. Where necessary different models or theoretical stances will be introduced and discussed in conjunction with these terms.

\section{Theoretical models: a brief introduction.}

A number of different models have been advanced to explain the common dissociation between implicit and explicit test performance. As noted by Schacter (1987) no single theoretical model can adequately explain all of the data but rather different theories explain subsets of the data better than others. The following is a brief introduction to these main theories (these will be expanded on in later sections).

The elaboration model suggests that priming effects in implicit memory tests are due to the automatic temporary activation of pre-existing representations or cognitive knowledge structures (Graf \& Mandler, 1984; Mandler, 1980). Once activated these representations can readily come to mind, but since this process does not associate an item as being part of a recent episode, this does not contribute to explicit test performance.

On the other hand elaboration associates a target representation to other information in memory. As a result elaborated memories have more associations with other information in memory than unelaborated representations and thus aid in retrieval during explicit memory tasks. Since elaboration is a context driven process, that is it associates a target representation with other related concepts, this will support explicit test performance. However this is of little benefit in implicit tests which typically do not require participants to engage in context driven retrieval processes.

Danion et al. (1991) note that the activation - elaboration framework is useful in that it provides a direct connection between explicit and implicit memory on the one hand and between effort demanding, that is elaborative and automatic or 'activation' processes on the other. As a result they suggest this 
can be of use in studying clinical groups who are characteristically impaired in tasks that require elaborative or effort demanding processes.

While the elaboration model can readily account for research which shows that priming decays rapidly in normals and amnesics under specific situations (Graf \& Mandler, 1984) and that the priming of pre-existing representations does not depend on elaborative processing (Jacoby \& Dallas, 1981) there are a number of areas that this theory has difficulty explaining. For instance some experiments have reported amnesic participants can show implicit memory for new information (Graf \& Schacter, 1985) while others have reported priming effects lasting for up to several months in normal participants (Graf \& Schacter, 1985, 1989; see also Roediger \& Blaxton, 1987).

The memory system model accounts for differences in test performance by referring to different underlying memory systems. One source of justification for this view is based on the principles of evolution and ontogeny (Schacter, 1987). It has been suggested that the development of human memory has involved the development of two (or more) memory systems, one available at birth and another at eight to ten months. This later system is associated with the recall not only of memories in general but also when the event occurred and other contextual details. It is thought that these two systems have developed with such specialisation that they have become functionally incompatible, that is one system is not able to be served by the other.

These hypothesised memory systems are drawn from the theoretical alternatives in the major divisions of human memory. For instance the episodic memory system (Tulving, 1972, 1983) is thought to be the basis for explicit memory while semantic memory accounts for implicit test performance. As an alternative Squire and Cohen (1984) suggest that explicit memory is a property of the declarative memory system while implicit test performance is attributed to the operation of a procedural memory system.

There are a number of theoretical difficulties with this view. Squire (1986) has suggested that procedural memory is responsible for all priming effects but evidence suggests that priming and skill learning can be dissociated 
experimentally. For instance Butters, Heindel and Salmon (1990) found that a group of amnesic participants showed impaired verbal priming but normal motor skill learning. Amnesics can also show implicit memory for newly acquired facts and vocabulary even when they show no explicit memory for this information (Schacter, 1985). Yet it is commonly assumed that the learning of new facts is the responsibility of the declarative system (which is assumed to support explicit test performance) rather than the procedural system. The episodic - semantic distinction has been characterised by a number of similar weaknesses to those outlined above.

Processing theories seek to explain test differences with reference to the nature of the overlap in the processes engaged in during both encoding and retrieval / testing. (Jacoby, 1983; Roediger \& Blaxton,1987). In brief this assumes that performance on a memory test benefits to the extent that the cognitive processes engaged at test overlap those used at encoding. Two main forms of processes are proposed to support human memory performance. Perceptual or data driven processes occur in response to the data presented in the test materials. Materials of this type typically engage perceptual processes and result in an orthographical memory of the stimuli. In contrast conceptual driven processes are those which primarily engage an analysis of meaning.

Typically the most common implicit tests, for example perceptual identification, word and stem fragment completion, draw primarily on perceptual processes and the most common explicit tests, for instance free recall draws primarily on conceptual processes. Dissociations are therefore attributed to the degree to which the processes required for test performance reinstate those which were used during encoding.

The processing view is also characterised by a number of problems in explaining the available data. It has been stated that elaborative study processing should not effect performance on perceptual implicit memory tasks such as stem completion (Roediger \& Blaxton, 1987). Some studies, however have shown that stem completion performance is to some extent dependant on elaboration (Schacter \& Graf, 1987). The processing view also has difficulty 
explaining the findings that priming can decay very rapidly and that some forms of priming effects depend on pre-existing representations in amnesic patients (Schacter \& Graf, 1986).

While the above theories have been presented as distinct entities more recently these models have started to move closer together. For instance Tulving and Schacter (1990) have stated that there is no necessary incompatibility between system and process theories. To date no 'grand theory' has emerged partly because individually each theory has made a distinct contribution and is able to explain aspects of the data which other theories are not able to (Schacter, 1987). However in more recent years the processing theory has received particular experimental attention largely because it is able to fit a reasonable amount of the available data (Roediger, Rajaram \& Srinivas, 1990). This theory will be expanded on in fuller detail in the next section.

\section{1 The processing model}

The processing model, as outlined by Roediger (1990) and Blaxton (1989) is an amalgam of insights gained from previous research (Kolers,1975,1979; Roediger, Weldon \& Challis, 1989). For instance it borrows from encoding specificity principles (Tulving,1983), transfer appropriate processing (Morris, Brandsford \& Franks,1977) and relies heavily on a set of context - no-context encoding operations outlined by Jacoby (1983).

The processing model is based on a number of principles (Srinivas \& Roediger, 1990), the most important being that a memory test will benefit to the extent that the processing operations performed at the time of test overlap those used at study / encoding. This is very similar to the logic of transfer appropriate processing (TAP). TAP asserts that the most appropriate learning strategy for a participant is one that most closely engages the memory processes used during test performance. For instance if a participant is required to perform a test which requires semantic processing the best memory transfer will be achieved from a semantic orienting task. 
The second principle also builds on the logic of TAP. This states that implicit and explicit tests typically require different forms of processing and therefore each test will benefit differentially according to the relative match between the processing operations performed at study and those used at test (Blaxton, 1989). By this reasoning performance on a memory test is expected to be facilitated leading to an increase in fluency and efficiency, to the extent that it engages the same or a similar set of cognitive operations used at encoding.

Processing operations can be divided into two major forms, perceptual and conceptual. Perceptual processes are those cognitive activities sensitive to and guided by variations in the physical, perceptual or 'surface' features of a stimulus/target (Jacoby, 1983; Roediger \& Blaxton,1987). Perceptual tests are specifically designed to engage one of the perceptual systems (usually vision) by presenting stimuli either rapidly or in a fragmented form, for example the perceptual identification test. Conceptual processes are those cognitive processes which are sensitive to and guided by the higher order, meaningful, contextual features within the stimulus/ target, for example elaboration, generation, imagery etc. (Roediger \& Blaxton, 1987). Conceptual tests involve an analysis of meaning (e.g. answering general knowledge questions) and as a result engage higher order processes for successful performance.

These two forms of processing occupy two separate continua (Blaxton,1989,1992; Schwartz, 1989) and as a result they need not trade off each other. Furthermore tests can be placed on a continuum from those which are primarily based on perceptual operations to those which require a high conceptual content for priming to be shown.

The majority of tests performed under implicit test instructions, for example word stem completion, perceptual identification, rely on the match between the surface features of the stimuli presented at test and the stimuli presented at encoding. Typically as this match is reduced priming is reduced. Tests which rely on a match between physical features are defined as perceptually driven tests. On the other hand most tests performed under explicit test instructions, for example free recall, rely on the encoded meaning 
of concepts (Blaxton, 1989) for the successful transfer of information between study and test.

Blaxton $(1989,1992)$ has pointed out that the read - generate encoding manipulation can be used to operationalise perceptual and conceptual processes at encoding. In the read condition participants are shown a word and asked to read this out, usually aloud (typically participants are also asked to keep looking at the word). By having participants read words this focuses attention on the physical features of words and this is assumed to engage primarily perceptual processes. In the generate condition the participants are asked to name (generate) a word based on a sentence cue. For this to occur the participant must engage in an analysis of meaning. For example participants are shown a sentence such as, "this flies and is an emblem of the United States" e_ . To correctly complete this the participant would say aloud "eagle". Here the target is not read and the generation procedure is assumed to engage more meaning based or conceptual processes.

It is a particular advantage to manipulate the relative contribution of perceptual and conceptual processes not only at test but also at encoding. By using this encoding manipulation this provides an additional check on the nature of the test in use (Blaxton, 1989). That is, perceptual tests can be defined as those in which studied items in the read condition produce better transfer, than those studied under generate conditions. The reverse can be used to define a conceptual test, that is better generate transfer.

While the read - generate encoding manipulation provides a measure of the contribution of respectively, perceptual and conceptual processes, it would be a mistake to describe this manipulation as representing process pure measures (Blaxton, 1989). For instance the transfer from the read encoding condition in a conceptual test is likely to involve a conceptual component as well (H. L. Roediger, personal communication, December 10, 1997). However the assumption would be that transfer from the generate condition would involve a higher conceptual component. As a result these measures can only 
be regarded as providing an approximate guide (although the use of an encoding manipulation is an improvement over having no guide at all).

Roediger et al. (1989) have suggested a number of converging operations as a basis for classifying tests as conceptually or perceptually driven (these include the use of the read - generate manipulations outlined above). Perceptual tests should be more effected by changes in surface information between study and test. For instance the presentation of pictures of objects at study and word fragments at test should hinder information transfer. On the other hand conceptual tests should be less effected by the manipulations of surface information but strongly effected by changes in conceptual elaboration, for instance the use of a LOP manipulation. In this later case greater priming should be expected from semantic study conditions than perceptual study conditions, since both the study and test operations draw primarily on conceptual processes. Indeed across a number of experiments (see Srnivas and Roediger, 1990) a number of tests, for instance the perceptual identification test have been shown to be primarily perceptual in nature while others, for instance the category association test have been shown to be primarily conceptual in nature.

\subsection{The processing versus memory systems models: the contribution of Blaxton.}

Blaxton (1989, 1992, 1995) has used the processing model as a basis for raising and testing a number of important insights. She pointed out that in the past the traditional memory system view and the processing view have been confounded. This is because experimenters used explicit tests that were primarily conceptual in nature and which were assumed to draw on the episodic memory system. These were compared with implicit tests which were primarily perceptual in nature and which were assumed to draw on the semantic memory system. As a result a clean comparison between the two views was not possible. 
In order to provide this comparison Blaxton (1989) suggested that it would be necessary to also include an implicit test that drew on conceptual processes and an explicit test that used perceptual processes. As a result Blaxton (1989: Expt. 1), designed a four-way matrix that included one test of each kind. Her two explicit tests were the semantic cued recall test (conceptual) and the graphemic cued recall test (perceptual). In the semantic cued recall test the participant is provided with a cue word that is similar in meaning to the target word read or generated during encoding. In the case of the graphemic cued recall test the participant was provided with a cue word that was similar in physical appearance to the target word. In both cases the participants were required to use these cues to recall target words.

The implicit tests were the word fragment test (perceptual) and answering general knowledge questions (conceptual). In this later case the participant was provided with a question (e.g. "what was the big bang said to have created?") for which the answer was usually a word that had earlier been encoded (e.g. "universe"). On this basis Blaxton (1989) was able to directly pit conceptual and perceptual versions of both implicit (semantic) and explicit (episodic) test versions.

During the encoding phase Blaxton (1989) used the read - generate encoding manipulation. Her results showed that dissociations were found between the conceptual and perceptual tests regardless of the implicit - explicit nature of these tests. For instance read - generate priming patterns were directly opposite for the graphemic cued recall test (which favoured transfer from the read condition) and the semantic cued recall test (which favoured generate transfer). This dissociation occurred despite the assumption that these tests should draw on the same episodic memory system.

In other words dissociations were found between two tests that were thought to tap the same memory system, for instance the episodic system. On the other hand dissociations were not found between tests which relied on the same type of process, that is the two perceptual tests consistently favoured read priming and the two conceptual tests consistently favoured generate priming. 
This finding led Blaxton (1989) to conclude that these results favoured the processing model over the memory systems model.

Although the processing account has had reasonable success explaining the results obtained from normal population samples, Blaxton (1992) noted that it was important to extend these findings to experiments using memory impaired participants. If a memory impairment led to a disruption in a memory system, for example the episodic system, then this should lead to a dissociation between a test which draws on that system and a test based on another unimpaired system, for instance the semantic memory system. On the other hand if a disruption occurred in a type of process, impairments should be found in tests which drew on that form of processing while tests which drew on other forms of processing will remain spared.

Blaxton (1992) examined this issue in a series of experiments with memory impaired participants with intractable temporal lobe epilepsy (TLE). Blaxton's assumption was that memory impaired participants would be impaired on tests which drew on conceptual processes. This impairment was mainly due to the greater likelihood that these 'higher order' resource demanding processes would be disrupted by a memory impairment. On the other hand perceptual tests should show minimal disruption largely due to the largely 'automatic' nature of this type of process.

Blaxton (1992:Exp. 1) used the same four tests and a very similar design to Blaxton (1989). She compared read - generate priming performance across her four tests and found that for her two conceptual tests there was no significant difference between read - generate priming. For the two perceptual tests a significant difference was found, this favouring the read encoding condition. Blaxton (1992) took the lack of conceptual transfer in her conceptual tests as evidence of a conceptual deficit. A conceptual deficit was defined as significantly poorer transfer in a condition /test designed to measure conceptual processing, for instance generate transfer in a conceptual test. Furthermore her dissociations were consistently found between the conceptual and perceptual tests rather than across her implicit and explicit tests. 
In sum Blaxton's $(1989,1992)$ series of experiments have emphasised the need to look beneath the labels 'implicit' and 'explicit' and take note of the processes operating behind these labels. Blaxton (1989) has shown that often test dissociations are best explained with reference to the type of processes engaged in between study and test rather than referring to differences in hypothesised memory systems.

\subsection{Problems with the processing model}

Despite the success of the processing model in predicting and explaining dissociations across a range of participants, more recent experiments have shown this model is not as straight forward as once thought. Blaxton (1989) noted that she had to use a 'bootstrap' technique to determine which tests were primarily perceptual or conceptually driven. That is, a test would have to be used in conjunction with an encoding manipulation (e.g. the read - generate manipulation) before that test could be judged to be primarily conceptual, perceptual or something in between. However following this initial step tests could be operationally defined for future experiments.

Blaxton (1989:Exp.3) found, however that once categorised, under certain conditions a test can behave in different ways. Blaxton found that when her typefaces varied between study and test, performance was higher in her imagery condition than in the read condition for her perceptual tests. She concluded that while these tests would normally be classified as primarily perceptual, depending on the conditions in use, they can also show a reasonable level of conceptual transfer. As a result of this finding Blaxton concluded that tests cannot be guaranteed to 'behave' or belong to one category across the total range of test conditions, all the time.

Likewise a number of other experiments have shown that a number of the assumptions held by processing theorists can be somewhat simplistic. For instance traditionally it was thought that Levels of Processing (LOP) manipulations had little impact on perceptual tests, but a robust effect on explicit 
tests (Hirshman et al., 1990). A number of experiments have challenged this assumption. In a typical LOP manipulation several different forms of processing type are used at encoding, for instance those that concentrate on higher order semantic processes and those that concentrate on physical or perceptual features. Tharpar and Greene (1994) found significant LOP effects in perceptual and conceptual implicit tests when stimuli were encoded using a blocked presentation. Challis and Brodbeck (1992) also found semantic encoding enhanced performance in a word fragment completion task, once again with blocked presentation. Tharpar and Greene concluded " at one time simple generalisations could be made, such as that levels of processing has no effect on implicit tests or on perceptual tests. Such simple generalisations no longer seem possible". (p. 678).

Graf and Ryan (1990) have also pointed out a number of problems with the processing model. For instance when the same test cues are used (combined with different instructions) dissociations can still be found between implicit and explicit tests even though both tests allow the same amount of perceptual processing (Graf \& Schacter, 1987). Others have also found that the traditional processing view does not always account for their data. Tharpar and Greene (1994) found LOP effects across both perceptual and conceptual implicit tests, while list order had no effect on the magnitude of the LOP effects in their explicit tests. In other words dissociations did not occur between the type of processing used but whether the test was implicit or explicit.

Even where conceptual and perceptual versions of explicit and implicit tests are used, at times dissociations are found only between the implicit and explicit tests, rather than between the different forms of processing. For instance this has been the outcome in a number of different experiments including those with schizophrenics (Schwartz, Rosse \& Deutsch (1993), the aged (Java \& Gardiner, 1991; Light \& Albertson, 1989) and those with Korsakoff syndrome (Vaidya, Gabrieli, Keane \& Monti (1995).

Maki (1995) has also raised the importance of including an analysis of task differences in understanding dissociations between tests. In a review of 
implicit memory priming in patients diagnosed with Alzheimer's disease, Maki noted that the data at times lacked internal consistency. She noted that not every participant comparison between perceptual and conceptual tests revealed a dissociation between these tests. Instead she suggested that a consideration of methodological differences may help explain these inconsistencies. For instance Maki noted that while many implicit conceptual tests revealed deficits in performance these tended to occur only when shallow forms of encoding were used, for example reading out a list of words. When participants were asked to use an encoding manipulation that encouraged deep semantic elaboration no deficit was found. Maki suggested that this could be due to the stronger memory trace created by a deeper level of encoding. As a result, differences in test design may explain why some tests record dissociations and others do not, rather than the operation of a conceptual deficit as such. Although she argues that a conceptual deficit can be a real phenomena, she does suggest that one needs a view that extends beyond conceptual / perceptual distinctions, but also makes consideration of the procedures used in measuring implicit memory effects. If this is not done researchers may attribute their results to the wrong source.

\subsection{The processing versus memory system models: time for a combined model?}

Recently the processing and memory systems view have started to move closer together. This move is partly in response to the problems reported above as well as a growing number of studies which suggest that human memory is not unitary, yet also support the distinction between conceptual and perceptual processing in describing test performance, for example Maki (1995); Gabrieli (1995).

Memory system theorists have begun to design models which attribute the operation of a distinct process within a memory sub-system. In a seminal paper Tulving and Schacter (1990) attributed implicit perceptual priming to the 
operation of a sub-system they described as the perceptual representation system (PRS). They proposed that this system existed separately yet could operate closely with other memory systems. For instance they proposed that the PRS was a pre-semantic system (as a result it couldn't undertake an analysis of meaning) and as such was capable of operating independently of the semantic memory system. Furthermore the PRS was relatively immune to the effects of drugs, ageing and clinical conditions which more readily effect other memory systems. It was proposed that the PRS is a critical system responsible for priming in word fragments, stems, perceptual identification, identifying non-verbal shapes, for instance drawings and novel visual objects.

Schacter (1992) readily admits that the semantic priming observed in a conceptual implicit test has very little to do with the operation of the PRS. Instead it was proposed that this reflects a process of semantic learning, that is the modification or adding of new information to semantic memory. Turning to explicit memory tasks, Schacter $(1992,1994)$ has proposed that the basis of priming in explicit conceptual tasks was the modification of episodic memory. While the exact basis of explicit perceptual priming remains unclear it was proposed that the PRS was able to feed into the episodic memory system and as a result provide a source for this type of memory transfer.

This revised model has a number of distinct advantages (from now on this updated model will be called the revised model as opposed to the original model). For instance it is now possible to consider the operation of distinct 'processes' within a memory system. This may help explain a number of the problems that can arise through examining the operation of a number of memory systems without considering the effect of different processing forms within those systems. For instance disrupted explicit conceptual recall and unimpaired conceptual priming could be explained with reference to impaired episodic memory and unimpaired semantic memory.

The increasing merger between these two models has also raised a number of questions. Schacter (1992) has noted that the dissociation between an implicit and explicit test is not sufficient in itself to postulate or prove a distinct 
memory system or sub-system, if this were the case the list would soon grow. Roediger (1990) has criticised the expansion of memory systems as a means of explaining test dissociations and points out that if rigorous criteria were applied many proposed 'memory systems' would not measure up. Roediger et al. (1990) have noted that the criteria used for postulating distinct memory systems, for example functional dissociation, independent neural pathways, stochastic independence and functional incompatibility are often not fully reliable by themselves. Schacter (1992) has suggested that it is important to have a basis for postulating different memory systems that are independent of dissociations observed in implicit - explicit memory experiments.

In the meantime most researchers agree that no one theory is able to account for all the data (Maki, 1995) and that a consideration of both theoretical positions, often in the same experiment should be the most useful future research strategy (Blaxton, 1995).

\subsection{Implicit - explicit memory research with clinical participants}

Following on from Warrington and Weiskrantz's (1968) landmark study, an increasing number of clinical groups have been investigated using an implicit - explicit test paradigm. These investigations have compared participants as diverse as the aged, participants with amnesia and other neurological conditions, those with a variety of psychiatric disorders and those under the influence of various forms of drugs.

Many investigators have continued to study amnesic populations largely because the brain lesions which characterise these participants can be used to help map the neural architecture of human memory (Keane, Gabrieli, Fennema, Growdon \& Corkin, 1991). For instance Cermak, Talbot, Chandler and Wolbarst (1985) examined alcoholic Korsakoff patients and matched controls on an implicit test of perceptual identification and an explicit test of recognition. The experimental group all had a history of chronic alcoholism leading to retrograde amnesia (difficulty recalling past events) of varying levels. As a result these 
participants were unable to recall everyday and current events. The control group consisted of chronic alcoholics. There was no evidence of neurological damage or psychiatric illness in these participants.

The results for the recognition test showed that there were no differences between groups when there was no delay between encoding and recognition. When a delay was introduced, the amnesic participants performed significantly worse than the control participants. The implicit test, on the other hand was found to be unimpaired regardless of whether this was delayed or presented immediately.

This pattern of intact implicit test performance and impaired explicit test performance has been repeated in numerous tests using amnesic participants of different aetiologies. For instance normal implicit test performance have been found in paradigms as diverse as measures of skill learning (Cohen \& Squire, 1980), classical conditioning (Weiskrantz \& Warrington, 1979) and repetition priming (Graf, Squire, \& Mandler, 1984). However this is not always the case and at times impaired priming is found on some implicit tasks, for example Cermak, Verfaellie and Letourneau (1993) found impaired priming in a test of degraded picture identification.

On occasions experimenters have chosen to compare a number of different participant groups within the same experiment rather than only comparing a single experimental and control group. By using the same experimental conditions direct comparisons can be more easily made between participants.

As an example, Butters, Heindel and Salmon (1990) reviewed a range of experiments which compared participants in the early stages of Alzheimers disease (AD), Huntington's disease (HD) and intact control participants. Butters et al. note that in the early stages of these diseases they manifest quite different patterns of brain dysfunction and associated symptomatology. In their review implicit tests of verbal and pictorial priming, physical skill learning and weightbiasing tasks were compared, usually within the same experiment. 
Overall the results were very consistent, participants with $A D$ were impaired on verbal and pictorial priming tasks, but normal on tests of physical skills and weight biasing tasks. In contrast participants with HD were impaired on the later tests, but showed normal levels of verbal and pictorial priming. A 'double dissociation' provides further evidence that implicit memory is not necessarily an unitary phenomena but that the same or different participant groups can reveal intact or impaired performance on different forms of implicit memory tests.

More recently a number of experiments have changed their test design to take into account the type of processes used at encoding and test. As a result a number of experiments found results which were supportive of the processing model (Maki, 1995), using aged or clinical participants. For example Light and Singh (1987) tested young and old participants across a range of implicit perceptual and explicit conceptual tests. They had the added advantage of using an encoding manipulation which was similar in nature to the read generate encoding manipulation. They found no differences in transfer in their perceptual tests, but impaired 'generate' transfer in their conceptual tests.

In contrast a number of experiments have compared conceptual test performance across a range of implicit and explicit tests yet found only a deficit in performance when intentional retrieval instructions were used, (see Java \& Gardiner, 1991; Light \& Albertson, 1989; Schwartz et al., 1993).

In sum a range of clinical groups have been compared in their implicit explicit test performance. While many of these experiments have shown intact implicit and impaired explicit test performance (and this tends to remain a typical finding), when a range of conditions, encoding instructions and tests are used this can reveal a different pattern of performance. 


\subsection{Depression and memory research (general).}

\section{Diagnostic criteria}

According to the Diagnostic and Statistical Manual fourth edition [DSM-IV] (American Psychiatric Association [APA], 1994) 'depression' is not an unitary phenomena but can exist in a variety of forms. For instance a major classification distinction is between bipolar depression (characterised by mood swings from a manic state to a depressive state) and unipolar depression which is not characterised by manic phases. Unipolar depression can be subdivided further into major depressive disorders, dysthymic disorder and depressive disorder not otherwise specified. DSM-IV lists a variety of symptoms to assist in the diagnosis of a major depressive disorder, these include depressed mood, markedly diminished interest in all, or almost all activities on a daily basis, significant weight loss or weight gain, fatigue or loss of energy nearly every day, among others. Diagnosis requires the presence of one or the other of the first two symptoms listed above and a further four or more symptoms should also be present over a two week period. These symptoms should also represent a change from previous functioning (there are a number of other diagnosis criteria, see page 327 \& 344, DSM-IV). Once diagnosed the disorder can be specified (among others) as mild, moderate or severe (with or without psychotic features) or in partial/ full remission. Major depression disorder is distinguished from dysthymic disorder largely on the basis of duration and how chronic the condition is. While in the former a variety of symptoms must be present for at least 2 weeks, in the latter symptoms must be present for more days than not over a 2 year period.

According to DSM-IV the prevalence of major depression disorder in community samples varies from 5 to $9 \%$ for women and 2 to $3 \%$ for men. Of particular interest to this study is the symptom of "diminished ability to think or concentrate, or indecisiveness, nearly every day, either by the participants 
account or as observed by others" (APA, 1994, p. 222). This suggests that depression can be marked by reduced cognitive/ memory function, indeed DSM-IV states:

"they (depressed individuals) may appear easily distracted or complain of memory difficulties...in elderly individuals with a major depressive episode, memory difficulties may be the chief complaint and may be mistaken for early signs of dementia. When the major depressive episode is successfully treated, the memory problems often fully abate" (APA, 1994,p. 322).

\section{Depression - memory research}

Numerous researchers have examined the nature of memory dysfunction in depression (see Brand \& Jolles, 1987; Richards \& Ruff, 1989) and have noted a number of deficits in both short and long term memory measures.

These deficits, also described as 'depressive deficits' can be defined as significantly worse depressive performance compared to control performance on a particular measure, for instance the free recall of words. Typically past depression - memory experiments have only used some form of explicit test measure, for example free recall. However as Bazin, Perruchet, de Bonis and Feline (1994) previously noted this represents only one aspect of memory performance that can be studied, that is implicit processes can be studied as well.

Those studies which have used an explicit measure have been reasonably conclusive. For instance Weingartner, Cohen, Murphy, Martello and Gerdt (1981) required their participants to encode a list of words using semantic and acoustic encoding conditions. In the former condition participants were asked to decide if a word was related in meaning to a stimulus word. In the later condition participants were asked to decide if the two words sounded the same. Depressed deficits were found in the free and cued recall tests, but 
only for words that had earlier been semantically encoded. They concluded that impairments were more marked in processing conditions which required the use of more elaborate encoding conditions, rather than shallow forms of processing.

Roy-Byrne, Weingarter, Bierer, Thompson and Post (1986) presented their participants with a list of word pairs (pen/book) and asked them to make one of four judgements about these pairs, for example was one word in the pair more or less valuable than the other? Later, participants were asked to recall as many words from the pairs as possible, (this was defined as the effort demanding task). Participants were then presented with each pair and were asked to recall which judgement they had made, (this task was defined as a more automatic task). They found that the depressed participants performed within a normal range on this automatic task and more poorly on the effort demanding task .

Watts and Sharrock (1987) found no significant impairment in a test of recognition but significant differences were found for their free recall and cued recall tests. They concluded that it made little difference to the depressive memory deficit whether participants were provided with retrieval cues (i.e. cued recall) or needed to generate their own retrieval cues (free recall).

In a major review of depression-memory research Hartlage, Alloy, Vasquez and Dykman (1993) reached the general conclusion that depression often significantly impairs effortful processing while it interferes very minimally with automatic processes. The degree of interference is determined by the relative difficulty of the task, the severity of the depression and the valence of the stimulus materials to be processed.

\subsection{Depression and implicit - explicit memory research.}

A number of studies have specifically looked at the differences between implicit and explicit test performance in depressed participants (refer Table 1.1). These can be divided into experiments which used 'depressed' university 
students, largely obtained on the basis of scores on a variety of self report inventories, and those which used clinical participants diagnosed in a clinical setting. Furthermore some of these studies looked at the occurrence of deficits using neutral word lists while others were primarily concerned with mood congruent memory (MCM). MCM refers to the tendency to recall information that is congruent with one's mood (Watkins et al., 1992). For instance if during a study phase participants were required to encode a list of positive, neutral and negative words, a MCM pattern would be shown if the depressed participants recalled more negative than positive words and the nondepressed control participants recalled more positive than negative words. Nearly all of these studies (apart from Watkins, Vache, Vernay, Muller \& Mathews,1996) used at least one test which can be defined as implicit and one test which can be defined as explicit.

\section{Experiments using neutral word lists}

To date the results for experiments using neutral word lists have been somewhat contradictory. Hertel and Hardin (1990:Exp.3) tested university students who were naturally depressed or 'induced' into a 'depressed' mood using the Velten mood induction procedure (Velten, 1968). Participants were placed into depressed and non depressed groups on the basis of scores obtained on a number of self report inventories including the Beck Depression Inventory (BDI). In the case of participants exposed to the Velten they were asked to read a series of statements, for instance "I am feeling sad today" designed to induce feelings of depression. Here the BDI was used as a check on the level of 'depression' experienced by these participants. All the experimental participants were then compared to a control group, who were experiencing a 'neutral' mood (confirmed by low scores on the BDI).

Hertel and Hardin (1990) used a spelling test as their measure of implicit retention. Most of the words used in this spelling test were homophones. A homophone is a word that can be spelt in at least two different ways with two 
distinct meanings, yet sounds the same when read aloud, for example dye, die. During the encoding phase participants were required to answer a series of questions designed to reflect the homophones less common meaning / spelling (this particular spelling was described as the target spelling). Each question was read out aloud, for example "what colour is a pear?", the participant spoke their answer.

During the test phase participants were given the spelling test which included many of the critical homophones heard during the encoding phase. For instance the participant would have heard the word 'pair', they could then choose to write this down as pair or pear (the latter being the target spelling). Priming was shown by an increase in the spelling of these old homophones in the unfamiliar way relative to the spelling used for a list of new homophones.

A test of recognition was used as the explicit test. The critical words for this test were also based on the unfamiliar spelling of the old homophones. Half of the participants were provided with a strategy to assist in their recognition decisions while the other half were not. This 'recognition strategy' consisted of a series of questions designed to help the participant make the connection between the words used in the encoding phase and the recognition task. As an example participants were asked to remember "if the word was in the set of questions in the first task and to try and recall a question with that word in it".

The results showed that there was no difference between groups in the implicit spelling test or in the explicit recognition of the participants provided with the strategy. When no strategy was provided the depressed participants recognised fewer of the old homophones than the mood induced participants, who in turn recognised less than the non depressed participants. While a trend was noted none of these differences were significant, although Hertel and Hardin (1990) note that typically deficits are not found in tests of recognition, when compared to tests of free recall (see also Weingartner et al., 1981).

In contrast to the above experiment, which used university students as participants, Danion et al. (1991) used clinical participants all of whom had been diagnosed with a major depression disorder (based on DSM-3 criteria) 
by a trained clinical professional. During the encoding phase participants were asked to remember a list of neutral words, each word being presented for 30 seconds. This phase was followed by an implicit word stem completion test (this used three letter word stems e.g. ele ). No differences were found in group performance for this test. Following the word stem completion test participants were given an explicit free recall test. The results for the free recall test showed that the control participants recalled significantly more target words than the depressed participants. Danion et al. (1991) concluded that the memory impairment observed in depression is limited to an impairment found only during cognitive processes which are effort demanding.

Elliot and Greene (1992) also tested participants sourced through a clinical treatment setting. All participants were diagnosed with a major depressive disorder by a trained mental health professional. During the encoding phase participants were shown a list of six letter words, presented at a rate of one word per five seconds. Half of these targets were then presented as an implicit word stem completion test (as described above). This implicit test was followed by an explicit cued recall test (based on the remaining words). Here participants were also presented with three letter word stems, these were used as the cues for explicit recall.

In phase two, participants heard a list of word pairs on cassette tape. For most of these word pairs the second word in each pair was a homophone. The first word in the pair was deliberately chosen to elicit the less common spelling of the second word, for example taxi-fare. The remaining pairs were nonhomophone word pairs interspersed among the critical homophone pairs. At the end of this encoding phase participants were given a spelling test (as an implicit test), which was based on the spelling of the critical homophones above as well as a number of non critical words which were interspersed to increase the acceptance of the task as a genuine spelling test. This test was scored by counting the number of homophones spelt according to the way they had been previously primed. Participants were then given an explicit free 
Table 1.1: Summary of previous depression / dysphoria implicit - explicit memory research

experiment/ test $\quad$ student (S) encoding task Clinical (C)

Probable MCM Deficit test status pattern

\section{Neutral word list}

Hertel and Hardin, exp 3 (1990)

implicit - homophone spelling

S answering

explicit - word recognition test

S questions

concep/mixed - no

Danion et al. (1991)

implicit - word stem

explicit - free recall

$\begin{array}{ll}\text { C } & \text { read word list } \\ \text { C } & \\ \text { C } & \text { read words } \\ \text { C } & \text { listen to words on } \\ \text { C } & \text { tape }\end{array}$

conceptual

no

Elliot and Greene (1992)

implicit - word stem completion

explicit - cued recall

implicit - homophone spelling

explicit - free recall tape

$\begin{array}{lll}\text { perceptual } & - & \text { no } \\ \text { conceptual } & - & \text { yes }\end{array}$

Mood congruent word list

Denny and Hunt (1992)

implicit - word fragment completion

explicit - free recall

C read \& rate words

C for life relevance

Watkins, Mathews, Williamson and Fuller (1992)

implicit - word fragment completion

explicit - cued recall

C read words \& imagine

Bazin et al. (1994)

implicit - word stems

explicit - word stems

C in a scene with word

Hertel (1994)

implicit - perceptual identification

explicit - free recal

C read list of words

C aloud

Ruiz and Gonzalez, exp 1 (1994)

implicit - word stems

explicit - free recall

C read words \& rate

C for 1. curvature /

2. emotion

S learn list of words

Bradley, Mogg and Williams (1995)

implicit - lexical decision test

explicit - free recall

C read words \& rate

C how often used

Bradley, Mogg and Millar (1996)

implicit - lexical decision test

implicit - lexical decision test

S read words \& rate

C how often used

Watkins, Vache, Vernay, Muller

\& Mathews (1996)

implicit - free association test perceptual no no

conceptual yes yes

perceptual no no

conceptual yes yes

perceptual no no

conceptual no yes

$\begin{array}{lll}\text { perceptual } & - & \text { yes } \\ \text { conceptual } & - & \text { yes } \\ \text { concep/mixed } & - & \text { yes } \\ \text { conceptual } & - & \text { yes }\end{array}$

perceptual - yes conceptual - yes

perceptual yes no conceptual yes yes

percep/mixed yes no conceptual yes no

percep/mixed yes no percep/mixed yes no 
recall test based on the homophones they heard during the spelling test. In scoring this test only the biased form of the homophone was counted as correct.

The results for the two explicit tests showed that the depressed participants recalled significantly less words than the control participants. Of greater interest was the finding that performance was also impaired for the two implicit tests. The authors concluded that clinical depression is capable of producing a global deficit that can effect both implicit and explicit memory processes.

A number of researchers have criticised the design used in this experiment. Bazin et al. (1994) note that since Elliot and Greene (1992) did not use a baseline it was more likely that participants would make use of an explicit recall strategy if they realised that some of the to be produced items were also from the study list. While this contamination is likely in any implicit experiment it is more likely to occur if each cue relates only to the words studied during the encoding phase. As a result, possible explicit contamination in the implicit tests could explain the obtained deficits on these tests. Bazin et al. (1994) also criticised the relative lack of power in this experiment (10 clinical participants were used). This could also be a possible source of this inconsistent result.

In sum: Of the three 'neutral word set' experiments, two experiments reported no significant differences between depressed and control group performance in the implicit tests. The one experiment which did report this difference (Elliot \& Greene, 1992) was based on participants sourced through a clinical setting and made use of the primarily perceptual word stem completion test and the more conceptual homophone spelling test.

Of the three experiments which used an explicit test measure (these were conceptually driven), two experiments reported that depressed participants performed significantly worse than the control participants. The one experiment which did not report a significant difference (Hertel \& Hardin, 1990: Expt. 3) was based on student rather than clinical participants. In all cases these experiments manipulated one variable at encoding, none used the read generate encoding manipulation at study. 


\section{Mood congruent memory tests}

Turning to the mood congruent studies there is a potential problem in directly comparing depressed participants who have been tested with a neutral word set and those tested using a MCM word set. In a congruent test not only is there presumably a depressive deficit inhibiting performance but also a mood congruent bias operating where by negative/ depressive words are more likely to be recalled (and similarly in control participants positive words are more likely to be recalled). By using word lists of varying valence, any depressed memory deficit is confounded with a mood congruent bias and this could be operating in opposition. As a result if a researcher is primarily interested in exploring depressive deficits the best means for this to be achieved is through the use of a neutral word list (Beck, 1967; Denny \& Hunt, 1992).

Ruiz-Caballero and Gonzalez (1994) tested dysphoric university students selected on the basis of BDI inventory scores. During Experiment 1 participants initially encoded a list of adjectives, half positive and half negative in valence. This was followed by an implicit word stem completion test and then an explicit free recall test. The implicit test revealed no overall differences between depressed and control participant performance, however a classic MCM pattern was found. For the free recall test, depressed participants recalled overall significantly less words than the control participants and a significant MCM pattern was revealed.

Watkins et al. (1996) used the implicit free association test to look at MCM biases in an university student sample (no explicit test was used). This test required participants to produce as many associations to a category cue as they could in 30 seconds. The results did not reveal a main effect of group, nor was any difference revealed in responding to the neutral cues, however a classic MCM pattern was found. 
A number of experiments also used participants sourced through a clinical setting. Several of these experiments contrasted an implicit test such as the word fragment completion test with an explicit test, for example free recall or cued recall. Of these Bazin et al. (1994); Denny and Hunt (1992), and Watkins et al. (1992) found no significant group differences or MCM pattern for their implicit test. Several found a significant group deficit for the explicit test (Bazin et al., 1994; Denny \& Hunt, 1992) and also a significant MCM pattern for this test (Denny \& Hunt, 1992; Watkins et al., 1992).

In contrast to the above Bradley, Mogg and Williams (1995) tested clinical participants with an implicit test (lexical decision) that included both sub threshold (below the level of awareness) and supra threshold conditions. The former was used as an index of automatic memory, the later as an index of strategic processes. The results showed that in clinical depression a significant MCM pattern was found under both conditions. Participants also participated in an unexpected explicit free recall test. This did not reveal a significant group effect, however depressed participants recalled more depressed words. The authors concluded that in depression, both implicit (automatic) and explicit (strategic) processes are biased in favour of depressive information.

In a follow up experiment, Bradley, Mogg and Millar (1996) compared mildly depressed/dysphoric students and clinical participants. The results showed that the dysphoric participants showed greater sub threshold priming of depression relevant words. Yet the clinical participants showed greater sub threshold and supra threshold priming of depression relevant words. Bradley et al. (1996) concluded that in dysphoria participants may be able to use strategic processes to counteract the effects of depression, that are unavailable to clinically depressed participants.

Finally Hertel (1994) required her participants to perform 2 rating tasks while they encoded a list of intermixed negative, positive and neutral words. Half of the words were rated for their emotional value to the participants and the other half for physical curvature (how round or angular the shape of the word appeared). Following the encoding phase participants were tested with an 
implicit perceptual identification task and then an explicit free recall test. Hertel found that for the explicit task the depressed participants recalled significantly less words overall (averaged over both tasks). The results for the implicit test showed that the depressed participants had longer reaction times for words rated for physical curvature and normal reaction times for words rated for emotional value. Hertel took this as further evidence that depressed participants suffer from inhibited control. Inhibited control was shown by the depressed participants lack of attention towards the curvature rating task. In contrast the depressed participants showed full attention to the emotion rating task (emotions often being of central concern to the depressed).

In sum: Of the nine experiments which used a word-set of mixed valence all nine experiments included an implicit test (these were virtually all perceptual in nature). Of these implicit tests, six experiments found a significant MCM bias, while none of the nine found any group differences in the overall recall of words. Of the three experiments which did not find an implicit MCM pattern these all used participants sourced from a clinical population. As a result most authors concluded that dysphoria / depression does not lead to an impairment in implicit memory, yet in many cases a MCM bias can be exhibited.

Six experiments also used an explicit test (these were all conceptual tests), of these five found a significant MCM pattern in responding, the one test which did not find this was sourced from a clinical population. Three experiments found that depressed participants recalled significantly less words than control participants. Of the experiments that did not find this, two used clinical participants and one student participants.

\section{Pharmaceutical drug use}

Two of the experiments discussed earlier also took into account the possible confounding effects of pharmaceutical drug use in samples drawn from clinical populations. Danion et al. (1991) compared medicated depressed participants with non-medicated depressed participants to a group of matched 
control participants. They found no significant difference between the depressed groups for the implicit or explicit tests, however when the depressed groups were combined they performed significantly worse on the explicit test. They concluded that the impairment was not due to a medication effect but rather to the effects of a relatively severe form of depression.

Bazin et al. (1994) retested their depressed participants towards the end of their hospital stay (most of the control participants were also re-tested). On the initial test a significant deficit was obtained for explicit test performance however on the rescreen no difference was found between the 2 groups. No differences were found for the implicit test during the initial test or during the subsequent rescreen. Even though most participants remained on psychotropic medication, a later rescreen showed that the overall level of the participants depressed mood had improved to a point where most participants were no longer classified as depressed. Bazin et al. concluded that the obtained deficit was explained best in reference to the participants mood state rather than factors such as medication use or to the hospitalisation experience itself.

\subsection{Models used to explain dissociations}

Researchers have cited a number of different theoretical models to explain the effect of depression on implicit - explicit memory. The activation versus elaboration model postulated by Graf and Mandler (1984) is referred to by a number of authors. Danion et al. (1991) suggest that this model is a good explanation for the 'typical' findings in depression - implicit memory research. For instance they suggest that explicit tests such as free recall are dependant on elaborative processes. As a result any depressive impairment is best explained by a disruption in conscious or effort demanding processes (these processes are viewed as more vulnerable to impairment in depression). An implicit task such as word completion is dependant on activation, a process which is theorised as automatic. They state that depression does not impair the 
automatic processes which implicit tests tap; this explains the dissociation between explicit (elaborative) and implicit (activation) tests.

Ruiz-Caballero and Gonzalez (1994) used the above model to explain their mood congruent findings. For instance in nondepressed participants positive representations were better elaborated while in depressed participants, negative representations were better elaborated. Similarly Watkins et al. (1992) suggest that a MCM bias is only found in explicit tests because mood congruent information have more associations to other items in memory than mood incongruent information. These associations result in greater numbers of mood congruent "memory handles" which assist in retrieval during an explicit test.

An alternative model put forward by Elliot and Greene (1992) is the processing model (Roediger \& Blaxton, 1987). Here it is thought that most implicit tasks require participants to be sensitive to the amount of surface level or perceptual processing carried out on the stimuli. On the other hand most explicit tasks such as free recall require participants to primarily attend to the meaningful or conceptual content of the stimulus material.

Although Blaxton (1992) did not make specific predictions about memory deficits in a mood state such as depression, she did infer that deficits in conceptual processing should be expected in participants who routinely exhibit deficits in memory performance. It would be reasonable to extend this prediction to include participants who are experiencing a depressed state since it is common to find impaired memory performance in these participants (Hartlage, Alloy, Vasquez \& Dykman, 1993). This being the case, in depression deficits should be routinely found in conceptual or mainly explicit tests, while perceptual or implicit tests remain spared. It is interesting that Elliot and Greene (1992) found deficits in both implicit and explicit tests, including their perceptual implicit test (this later finding is in contrast to Blaxton, 1992). They suggest that depression may produce a more global cognitive impairment and as a result interfere with both the surface level and meaningful aspects of stimuli.

Watkins et al. (1996) used the processing model as a basis for examining MCM biases in implicit memory. They claimed that previous attempts to find a 
MCM bias in implicit memory tests have failed because these tests were perceptually based and not conceptually based. Watkins et al. did demonstrate a MCM bias using a conceptual word association test and claimed this was found since the test tapped word meaning rather than just perceptual characteristics. However two previous studies have found a significant MCM bias with perceptually based implicit tests, which is problematic for the claim that a MCM bias will only be found on a conceptual implicit test.

In the wider implicit memory literature an alternative model is the memory systems model. The original version of this model suggests that most explicit memory tasks rely on episodic memory (which is disrupted by depression, Schacter, 1994) while implicit memory tasks rely on semantic memory which is largely spared in depression. It is interesting that none of the researchers have cited this model, although this may in part reflect that this model is often cited by researchers studying participants with identifiable brain damage (e.g. Cermak, Verfaellie \& Chase, 1995).

The resource allocation versus cognitive initiative model

Another influential model is the resource allocation model (Ellis \& Ashbrook, 1988; Ellis, Thomas \& Rodriguez, 1984) which partly draws upon Hasher and Zacks (1988) distinction between automatic and effortful memory. Ellis and Ashbrooks (1988) model is an useful adjunct to the processing model in that while this model assumes an impairment in conceptual processes it does not suggest a mechanism to explain how this occurs (Calesimo, 1994). The resource allocation model is one possible means of doing this, the implication being that conceptual processing requires greater capacity than perceptual processing.

Ellis and Ashbrook (1988) propose that there is a limited pool of available capacity for cognitive operations. Furthermore all tasks require the allocation of a measure of this limited capacity. They put forward a number of assumptions based on this basic view; a depressed mood state will tie up some 
of this capacity; mild mood states should have little impact on performance while intense mood states are highly likely to lead to a severe reduction in available cognitive capacity. Secondly, the encoding of information in everyday tasks (and many 'typical' lab experiments) will always require some allocation of capacity, that is they will not normally be performed at an automatic level. Thirdly memory performance is positively correlated with the degree of cognitive effort allocated to a given task with high degrees of effort correlated with superior performance.

They also add two additional assumptions to this, each of which are by definition irrelevant to the task at hand and can therefore lead to reduced test performance. Firstly a depressed mood state may increase the amount of task irrelevant processing (any class of processing activities which do not support the establishment of relevant features of a memory representation). Secondly participants can engage in extra task processing (extra cognitive activities such as worry, which reduce processing capacity).

On the basis of these assumptions Ellis and Ashbrook (1988) propose a number of predictions; firstly that a depressed mood state will reduce performance in a variety of cognitive operations such as memory with the greatest impairment likely to occur in unorganised, low meaning tasks. For instance higher recall would be expected from a list of words which shared a number of distinct category characteristics, for example four legged animals, household furniture. Less recall would be expected if the words were unrelated. As well, the inhibiting effects of a depressed mood state will be greater when the encoding demands are more difficult or demanding. The stronger the intensity of the depression the more likely that it will disrupt performance on a task. Ellis and Ashbrook contend that although a number of studies do not support their predictions, these can be explained with reference to the low intensity of the mood state (see also Potts, Camp \& Coyne, 1989) or the low minimal effort which the task requires, although they do acknowledge that there are a number of inconsistent studies which do not fit the model (see Clark \& Teasdale, 1985). 
Hertel (1994a,1994b) and her colleagues (Hertel \& Hardin, 1990) have contrasted the resource allocation model with a model which predicts a lack of cognitive initiative in depressed participants. The cognitive initiative model suggests that depressed persons may at times experience difficulties in monitoring and self sustained attention (Hertel \& Milan, 1994) but they are capable of performing resource demanding tasks when guided to use procedures which nondepressed participants perform on their own initiative. This poor initiative is more likely to be demonstrated in tests where the spontaneous use of strategies is not well controlled by the requirements of the test. While control participants spontaneously engage procedures which are not part of the instructions, they suggest that depressed participants are more likely to do only what they are told.

Hertel and her colleagues have shown that clinically depressed and dysphoric persons could perform at normal levels when experimental manipulations held their attention to the task (Hertel \& Rude, 1991) and 'asked' participants to monitor the relevance of what they did during the encoding phase (Hertel \& Hardin, 1990). Hertel and Rude note that while depressive deficits were commonly found in an undirected condition, this was eliminated following a task that focused attention during learning. Furthermore this improvement cannot be attributed to a reduced demand on cognitive resources, since the 'directed' task that eliminated the deficit was no less demanding than the undirected condition.

Hertel and Rude (1991) have also noted that their findings do not always demonstrate that depressed and non-depressed participants have comparable amounts of cognitive resources available. That is their depressed participants exhibited longer latencies on a recall task. A longer latency could suggest that depressed participants were thinking harder about the task materials without a corresponding benefit in recall. In response to this they have proposed a weaker version of the resource allocation theory. This proposes that depressed participants perform poorly on many cognitive tasks because they allocate fewer resources to these tasks largely due to a lack of initiative or a general 
state of inhibition (this also draws upon the work of Hasher \& Zack, 1988; see also, Kuhle \& Helle, 1986).

Ellis (1990) has more recently defended the resource allocation model against some of the criticisms made by Hertel and Hardin (1990). Although Ellis welcomes the initiative hypothesis in that it identifies a specific locus of the effect of depression, he criticises the direct comparison between a initiative and a resource allocation hypothesis. The resource allocation hypothesis does not place constraints on where deficits in performance may occur. Ellis suggests that these are not necessarily competing explanations since the degree of initiative shown in activating a process can itself be the outcome of a capacity allocation.

In sum, a number of different models have been used to account for implicit - explicit dissociations in depressed participants. A number of researchers have used the activation - elaboration model to account for deficits in depressed participant performance and the operation of MCM biases. Others have made reference to the processing model as an explanation for both deficits and MCM biases. Finally others have contrasted the resource allocation model and the cognitive initiative model to explain depressive deficits.

\subsection{The processing model and depression - implicit memory research.}

As previously outlined very few experiments have examined the occurrence of depressive deficits in implicit / explicit tests of memory using a neutral word list. The majority of these experiments have found the 'typical' finding of intact implicit memory and impaired explicit test performance. However most of these experiments have compared single implicit and explicit tests and have not manipulated or measured different forms of processing at either encoding and test.

In the past a variety of models, including the processing model, have been put forward as an explanation for test dissociations. In more recent years 
the processing model has emerged as a dominant model in implicit memory research, yet to date it has been used in part as an experimental model to research depressive deficits in implicit memory research.

The processing model is useful in that it takes note of a number of the design problems that have effected past implicit memory research. For instance there is the potential problem of applying the results found from one measure of memory, for example an implicit test and generalising these to all tests in that category (Roediger \& McDermott, 1992). This is a particular problem when the 'traditional' test design of comparing one implicit test with one explicit test is used. Roediger and McDermott (1992) note that since explicit tests can be dissociated as a function of experimental variables (this also applies for implicit tests) one cannot conclude that all the same class of test will behave in the same way.

Roediger and McDermott (1992) have also noted the difficulty in comparing results between experiments and between labs. When this is done this can result in the comparison of experiments which differ on many dimensions apart from the type of test used. For example, differences in staff, participants, physical environment, as well as possibly language and custom. The use of multiple tests/ study manipulations within the same experiment will help get around this problem (Rajaram \& Roediger, 1993).

As a result of these problems Roediger (1990) has recommended that one needs to manipulate independent variables on at least 2 tests that are thought to tap the same construct, for example 2 episodic tests or 2 semantic tests. If the effects of the independent variables is the same within each class of test then greater support is found for the theoretical distinction. At the same time such a strategy allows for the possibility that dissociations can be found within a class of test thus suggesting that no single theoretical construct can explain all implicit or explicit test performance.

The present study will examine the occurrence of dissociations in depressed participants using a variety of implicit and explicit tests that will vary in their processing requirements. The manipulation of process type will also 
occur at both encoding and test. This will have several important advantages. For instance it will enable an examination of the role of specific processes in explaining test dissociations in depressed participants. By using a design similar to Blaxton (1992) it will also be possible to extend the processing versus systems debate to a new participant group, namely depressed participants. Finally this series of experiments will involve greater experimental complexity than previously attempted with depressed participants. It is hoped that this will lead to more precise explanations and a higher degree of theoretical assurance. 


\subsection{Experiment 1}

An analysis of previous implicit / explicit memory research with depressed participants has shown that these experiments confounded process type (conceptual or perceptual) with the particular memory system (episodic or semantic) thought to support test performance. While this confound remains it is difficult to state which process and / or system best accounts for test performance and which of these are effected by depressive impairments.

In Experiment 1, a series of tests were included which compared depressed and control group performance on perceptual and conceptual versions of implicit / explicit tests. A design similar to Blaxton (1989: Expt. 1) was used (also similar to Blaxton, 1992). The read - generate encoding task was used at study to operationalise perceptual and conceptual processes during encoding. In order to unconfound the effects of mood congruency and depressive deficits a neutral word list was used in this experiment.

For this experiment several changes were made to the design used by Blaxton (1989). A category association test replaced a general knowledge question test since it was thought that in the later test the results may be particularly influenced by the individual variation in the participants ability to answer general knowledge questions. Blaxton's (1989) participants were given only one out of five possible tests. Due to the relative difficulty in obtaining depressed participants this was changed so that each participant undertook two out of four possible tests (an implicit task followed by an explicit task). Finally an interpolated task was included between the end of the study period and the start of the implicit task. This was included to reduce the opportunity for covert rehearsal and increase the likelihood that the implicit task will be viewed as just another in a series of paper and pencil tasks.

Apart from the change to the implicit conceptual task above, the same tests used by Blaxton (1989) were chosen for the other three combinations on the matrix. These were; word fragment completion (implicit - perceptual), 
graphemic cued recall ( explicit - perceptual) and semantic cued recall ( explicit - conceptually driven). Blaxtons (1989) test results as well as more recent experiments using a read - generate manipulation have shown that these tests were predominantly perceptual or conceptually driven as appropriate. For instance the word fragment (Blaxton, 1992) and graphemic cued recall (Blaxton, 1992) tests have been shown to be primarily perceptual in nature. Likewise the semantic cued recall (Blaxton, 1992; Cermack, Verfaellie \& Chase, 1995; Java \& Gardiner, 1991) and category association (Srinivas \& Roediger,1990) tests have been shown to be primarily conceptual in nature.

To date only one experiment has examined deficits in implicit and explicit tests, using a neutral word list with student participants (Hertel \& Hardin, 1990). While there has been some caution with the use of student participants (Hasher, Rose, Zacks, Sanft \& Doren, 1985), many experiments have obtained significant deficits with the use of students, including an implicit - explicit MCM study (Ruiz-Caballero \& Gonzalez,1994). For Experiment 1 student participants were recruited using criteria very similar to that used by Hertel and Hardin (1990); Hertel and Milan (1994); and Ruiz-Caballero and Gonzalez (1994).

For the processing model to be supported impairments should be found in both conceptual tests, while both perceptual tests should remain unimpaired despite the implicit - explicit nature of these tests. To date only aspects of the processing model have been tested using depressed participants. A number of previous experiments have found significant depressive deficits in tests that were both explicit and conceptual (Danion et al., 1991; Elliot \& Greene, 1992; Hertel \& Hardin, 1990). Likewise a number of experiments have used depressed participants and found no impairments in the implicit - perceptual tests that they have used, for example Danion et al. (1991) and Denny and Hunt (1992). However none of these experiments used a read - generate encoding manipulation at test to confirm their perceptual - conceptual status or to provide a guide on the contribution of different processing forms during test performance. 
The question of whether a deficit would occur in an implicit conceptual test with depressed participants is less certain partly because to date the few implicit tests using conceptual processes have been found to be normal (Watkins et al., 1996) or impaired (Elliot \& Greene, 1992) in depressed participants. The question as to whether explicit perceptual tests will remain unimpaired in depression has not been addressed by past research.

Should the range of impairments described above not be found in Experiment 1 this would be problematic for the processing model. However this range of impairments has been found across a variety of clinical groups such as the aged (Light \& Singh, 1987) and Alzheimer's patients (Maki, 1995), using a number of similar experiments to those proposed for Experiment 1. In order for the processing model to be supported, the depressed participants should perform normally on both perceptual tasks and they should be impaired on both conceptual tasks irrespective of the test instructions.

To show this Experiment 1 compared read - generate performance within each of the tests. Lower depressed participant transfer in the more conceptual 'generate' condition for both the implicit and explicit conceptual tests was predicted. This was because the generate condition is an index of conceptual transfer, a process which is more likely to be impaired in depressed participants (H. L. Roediger, personal communication, December 10, 1997).

No difference was expected in read performance in the conceptual tests, largely because priming from this condition is usually quite small, making it difficult to reveal differences between participant groups $(H$. L. Roediger, personal communication, December 10, 1997). No significant differences in performance should be found in the perceptual tests, despite the implicit explicit nature of these tests.

A number of implicit - explicit memory experiments have contrasted the processing and memory systems models using depressed (Danion et al., 1991) or clinical participants (Java \& Gardiner, 1991; Light \& Albertson, 1989; Schwartz et al., 1993) and found that it is the nature of the retrieval instructions which dictate the appearance of a deficit. For the original memory systems 
model to be supported in Experiment 1, significant impairments in depressed participant performance should be found in both explicit tests, while the implicit tests will be unimpaired. The original memory systems model does not make specific predictions about types of processes, as a result an impairment could be found in one or both of these processes, provided the impairment is restricted to explicit test performance.

\subsection{Method}

\section{Participants and assignment}

\section{Criteria}

Universal criteria for participation included age ranging from 17 to 50 , minimum of three years secondary education, English as a first language, no self reported difficulties in reading and writing and no uncorrected difficulties in vision.

The criteria for contacting participants to participate in the laboratory experiment was, for the experimental group a score of 15 or above on the Beck Depression Inventory ( Beck, 1970; Beck, Ward, Mendelson, Mock, \& Erbaugh, 1961) and for the control group, a BDI score of five or below. During the experimental session participants completed a second $\mathrm{BDI}$, if the score fell below nine they were removed from the experimental group.

\section{$\underline{\text { Recruitment }}$}

The BDI was distributed to two introductory calculus classes (400 plus students). It was emphasised that students were not required to fill in the BDI and that participation in part two was voluntary. After scoring the BDI's, the completed BDI's were split into several groups according to the criteria outlined above. 
Approximately four to five days after completing the BDI students who scored over 15 were contacted and asked to participate in a further study with the principle investigator. Once several experimental participants had been tested those who had scored under five were contacted and asked to take part in a further study. The control group were matched for age and education levels. For each experiment 'educational' level was calculated by totalling the participants secondary school and university education. This was calculated to the nearest year and month. Participant testing occurred over a two to three week period.

\section{Final assignment.}

Eighteen experimental participants were tested but two were removed since their scores had dropped below nine on the second completion of the $\mathrm{BDI}$. As a result 16 participants were included in the experimental group and sixteen matched control participants were also tested. $t$ tests showed that the mean age for the experimental group $(\bar{X}=21.87, \underline{S D}=5.69)$ and the control group $(\bar{X}=22.18, \underline{S D}=5.08)$ were not significantly different $(\underline{p}>0.05)$. The mean educational level for the experimental group $(\bar{X}=6.60)$ and the control group $(\bar{X}=6.28)$ were not significantly different $(\underline{p}>0.05)$.

\section{Memory tests and Materials}

All instructions and stimuli were presented on a Silicon IBM clone computer. Stimuli were presented in black against a white screen. Target words were taken from lists used in a series of earlier memory experiments by Blaxton (1989, 1992). These words were checked against a New Zealand (NZ) written corpus of English (Bauer, 1993) to ensure they were relevant for NZ participants. Where words were not relevant or did not meet the test criteria outlined below new words were added until a final pool of 96 words was chosen. All words were chosen for a neutral emotional tone and were drawn 
from a list of previously rated words (Brown \& Ure, 1969) or were rated using student volunteers. Students were asked to indicate, using a seven point Likert scale, the affective quality of the words. Only words receiving $90 \%$ agreement were included in the final pool.

The word pool was split into two lists of 48 words each, which were named list $A$ and list $B$. Within each of these lists three blocks of 16 words were developed. All words used in Experiment 1 were between four and nine letters in length and varied in frequency from four to 491 occurrences per million. Statistical analysis was used to ensure that there were no significant differences in word length or frequency between lists or blocks.

During each study phase one block was used for one of the encoding tasks, e.g. the read task, another block for the generate task and the final block was 'held back' and used as a baseline during the subsequent test phase. The order and combination of block and encoding task was counterbalanced across subjects.

The read task required participants to read out a list of words, while the generate task required participants to guess a word based on a letter at the end of an associated sentence, for example this is large and grey and has a trunk E___ (elephant). Norming work using student volunteers was used to ensure $95 \%$ correct completion of generate sentences before they were used. The use of the different blocks with the 2 encoding tasks and the baseline was counterbalanced across participants.

During encoding, items were read, or generated, in the same order within blocks (this was to facilitate the scoring of correct read and generate completions). Targets that were associated with the implicit test were always shown first. That is during the read task the read block which primed the implicit task was shown first and then the read block which was associated with the explicit task was presented. The order of presentation of the read and generate tests was counterbalanced across participants. 
Four memory tests were developed, two of which were implicit (word fragment completion and category name production) and two explicit (semantic and graphemic cued recall).

The word fragment completion test required participants to fill in the missing letters of word fragments, for instance e_e_h_nt (for elephant) in order to complete the first real word that came to mind. Norming work (in all cases using university undergraduate students) was used such that each fragment was correctly completed $30 \%$ of the time. The category association test required participants to write down examples of a specific category, for example animal, pig, deer, rabbit etc. Norming work was done to ensure that the correct target was associated with a category label at least $30 \%$ of the time.

The graphemic cued recall test presented cues which were similar in physical appearance to target words but had no similarity in meaning. For example for the target word "computer", the graphemic cue was "commuter". Participants were asked to use the cue to recall words from the encoding list which were similar in appearance. The semantic cued recall test presented cues which were similar in meaning to targets but had no physical similarity. For example for the target word "copper" the semantic cue was "bronze". Participants were asked to use the cue to recall words from the encoding list which were similar in meaning. Items to be presented as cues on the cued recall tests were normed by having participants search through lists containing targets and cues and matching them for semantic and graphemic similarity. An item was only used if $95 \%$ agreement was obtained.

Following the encoding task each participant completed one implicit test (which was always presented first) and then an explicit test. Each test was linked to a specific list, for instance the implicit test drew on the three blocks in list $\mathrm{A}$ and the explicit test on the three blocks in list $\mathrm{B}$. The order of presentation of lists and the tests associated with each list was counterbalanced across participants. The presentation of the implicit test and the explicit test was counterbalanced across participants, for example out of the four tests, each participant performed two tests, one implicit and one explicit. At test, in all cases 
the stimuli presented was a cue for a word from a read, generate, or baseline block. Thus by the end of the test 42 cue words were presented to the participant each of which related to a word that was previously read or generated or held back as a baseline word. During scoring the number of correct cue completions were added up for read, generated and baseline words.

For each of the four tests two booklets were made up (one based on list $A$ words and the other on list B words) containing relevant cues for the participant. Each booklet contained a different random order of the appropriate list (although the first three words in the implicit tests were always baseline words). The same font was used throughout the experiment on both the computer and in the booklets.

Participants were also required to fill in a participant question sheet. This asked the participants name, age, and the number of years of secondary and tertiary education. Participants also indicated their sex, whether English was their first language, whether they had any problems with reading and writing and that they had normal or corrected eyesight. Participants signed a participant consent form, this included a brief description of the experiment and the participants rights during and after the experiment. Although this stated that the experiment would end with a test of memory the exact nature of this test was not stipulated, the implicit tests were described as paper and pencil tests of thinking abilities.

The BDI was used as a measure to check the levels of depression experienced by control and experimental participants. This is the most common scale used to measure depression levels in experiments such as this one and studies have shown that the BDI has good reliability and validity when used with an university population (Bumberry, Oliver \& McClure, 1978) and with hospitalised adolescent populations (Strober, Green \& Carlson, 1981). 
Participants were tested individually in a quiet private room. After completing the participant question sheet and the ethical approval form any concerns were dealt with and the experiment began. Participants were asked to sit in front of an IBM computer where a welcome message told them that they would be asked to take part in a number of tasks. The nature of these tasks was not specified. This was followed by the instructions for the read or generate task.

In the read task the instructions stated,

"Shortly you will see a row of $X ' s(X X X)$ in the middle of the screen. This row of X's will then be replaced by a word. Please read this word out aloud. After six seconds the word will be replaced by another row of X's and a new trial will begin. Please remember to read the word out aloud each time. Before this part of the experiment begins there will be three practice trials. As soon as you are ready to begin please push the space bar".

In the generate task the instructions stated,

" Shortly you will see a sentence which will finish with a single letter. The sentence will be displayed on screen for six seconds. At the end of six seconds the sentence will disappear and you will hear a beep. This sentence will be completed by a single word and you must work out what this word is. This word will always begin with the letter at the end of the sentence. As soon as you know what the word is please state what it is aloud. Do not wait for the five seconds to be up before you state what the word is. If you produce the wrong word you will be told what the correct word is. If after six seconds you cannot work out what the word is you will be told the correct word. At the end of each trial you must push the space bar to bring up a new sentence. Before this part of the experiment begins there will be three practice trials. As soon as you are ready to begin please push the space bar".

At the conclusion of both read and generate practice trials a message stated, 
"as soon as you are ready please push the space bar and the trials will begin. A message will tell you that the trials are complete".

At the conclusion of the first generate sentence or read block there was a 15 second pause which was followed by the second generate sentence or read block words. At the conclusion of the second block of words to be read or generated a message stated,

"this part of the experiment is now complete. Please tell the experimenter".

During the generate task the experimenter sat near the participant with a list of correct generate sentence completions. The experimenter ticked correct replies and noted when the participant was unable to guess the correct completion. If this was the case the participant was told the correct answer.

After the first encoding task there was a 15 second pause before the second encoding task. After both encoding tasks the participant was immediately asked to take part in an interpolated task designed to cut down on covert rehearsal of target words. Participants were asked to write down as many New Zealand towns and cities as they could think of in 2 minutes. The interpolated task was followed by the instructions for the implicit task which was then followed by the instructions for the explicit task. All instructions were presented on page one of a 'task booklet' which included three examples and three practice trials. In all cases the experimenter went through the instructions with the participant and answered any questions before the participant began the experiment.

In the word fragment completion test the instructions stated,

"Please do not look through this booklet until you have read the instructions below. Please find below three examples of a word fragment. A word fragment is a word with some missing letters. Your task is to study each word fragment and to complete it with a real word. Please fill in the gaps with the FIRST real word that comes to mind with letters to make a word. Please spend twenty seconds studying each word fragment. All fragments are presented in lower case. To assist you in this if you press the read button on 
the computer it will beep every 20 seconds. To stop the beeping press the space bar, to start it again press the red button. While you are studying a fragment please cover up the remaining fragments with the guide sheet tucked below. Once you move on to a new fragment do not go back to any uncompleted fragments. If you complete a fragment within 20 seconds you are welcome to move on, but you will need to restart the timer. To do this push the space bar, then the red button. Please take a minute to study the three practise words below (three practise examples). When you are ready to begin please turn the page and leave the fragments covered with the guide sheet. Press the red button and start".

In the category association test the instructions stated,

"Please do not look through this booklet until you have read the instructions below. Please find below three examples of a category label. A category is a name for a group of objects or things. For instance the category 'flower' is a label for all objects which are flowers, for example gladioli, geranium etc. In this task you will be given a category label and you are asked to produce as many examples as you can of that category in 25 seconds. To assist you in this if you press the green button on the computer it will beep every 25 seconds. To stop the beeping, press the space bar, to start it again press the green button. While you are working on a category label cover up the remaining category labels with the guide sheet tucked below. Once you move onto a new category label please do not go back to any uncompleted categories. You may wish to start by practising on the three category labels below (three examples). Turn the page and make sure all the categories are covered with the guide sheet. Press the green button and start".

An extra five seconds was allowed for this task to allow for the completion of multiple entries.

In the graphemic cued recall test the instructions stated,

"Please do not look through this booklet until you have read the instructions below. Please find below three examples of a graphemic cue. A graphemic cue is a word that looks very similar in appearance to another word 
(three examples). Earlier in the session you read aloud a list of words either by reading them on a list or producing the word based on a sentence cue. Your task is to use the graphemic cues to try and recall the words you read aloud and produced from the sentence cues. Each graphemic cue looks similar to the words you read aloud or produced before, but none have any resemblance in terms of meaning. Please spend 20 seconds studying each graphemic cue. If you work out a word please write it down next to the graphemic cue. To assist you in the timing if you press the red button on the computer it will beep every 20 seconds. To stop the beeping, press the space bar, to start it again press the red button. While you are studying a graphemic cue please cover up the remaining cues with the guide sheet tucked below. Please note there may be some cues that are not similar to any of the words you studied earlier. Feel free to take a guess if these cues reminds you of a real word. If you complete the task in less than 20 seconds feel free to move to the next cue, however you will need to re-set the timer. To do this, please push the space bar, then push the red button. When you are ready to begin please turn the page and cover the cues with the guide sheet. Press the red button and start".

The semantic cued recall instructions stated,

"Please do not look through this booklet until you have read the instructions below. Please find below three examples of a semantic cue (three examples). A semantic cue is a word that is very similar in meaning to another word. Earlier in the session you read aloud a list of words either by reading them on a list or producing the word based on a sentence cue. Your task is to use the semantic cues to try and recall the words you read aloud or produced from the sentences. Each semantic cue looks similar to the words you read aloud before, but none have any resemblance in terms of the way the words look. Please spend 20 seconds studying each semantic cue. If you work out a word please write it down next to the semantic cue".

At this stage the instructions in regards to the timer, guide sheet etc. were the same as above (although changed to reflect the semantic nature of the test). 
At the conclusion of the explicit task the experimental group were left alone and asked to fill in the BDI and place it in an envelope. This was followed by a short debrief. Control participants were asked to fill in the BDI before the encoding session, they were also given a debrief at the end of the session. While control and experimental participants completed the BDI at different times this procedure was based on that used by Hertal and Hardin (1990) who noted that control participant scores showed some variation. Hertal and Hardin (1990) did not note any group effect through the use of this procedure.

Participants were paid $\$ 5$ at the conclusion of the experiment. The experimenter sat away from the participant during the test phases but remained in the vicinity of the participant to ensure that all test instructions were being followed. The experiment lasted between 40 and 50 minutes depending on the combination of conditions.

\subsection{Results}

Despite extensive norming it was found that a number of cues could match more than one completion (described as non-strict data). For instance the correct completion for the category association cue 'indoor toy' was puzzle. Yet it was found that this at times cued 'computer' from the general study list. Preliminary analysis compared the strict and non-strict data which showed that the differences in completions were not significant $(p>0.05)$. As a result analysis has been based on the non-strict data.

An analysis of the $\underline{t}$ test performed on the BDI scores for the depressed $(\bar{X}=14.75, \underline{S D}=3.47)$ and control $(\bar{X}=1.06, \underline{S D}=1.43)$ groups showed that the two groups were significantly different, $\mathrm{t}(30)=14.56, \mathrm{p}<0.001$.

Comparisons were made on the differences between list $A$ and list $B$ non-strict completions, for each type of test. Two of the $24 \mathrm{t}$ tests reached significance at the 0.05 level, both were from the control group for the explicit semantic cued recall condition. As a result the $A$ and $B$ list completions were collapsed into single non-strict scores for further analysis. 
Table 2.1 summarises the means for correct encoding (generate sentence completions) for the two groups for list A and list B. A 2 (Group: depressed and non-depressed) $\times 3$ (Block) ANOVA was performed on data for list $A$ and showed no significant main effect of Group ( $\underline{p}>0.05$ ), no main effect of Block, $(\underline{p}>0.05)$ and no interaction between Group X Block ( $\underline{p}>0.05)$. A 2 (Group) X 3 (Block) ANOVA on the generate sentence completions for list B showed no main effect of Group ( $\underline{p}>0.05)$, no main effect of Block ( $\underline{p}>0.05)$ and no interaction between Group X Block, $(\underline{p}>0.05)$.

A t test was also performed on the interpolated towns and cities task and found no difference between the 2 groups ( $p>0.05$ ) for this task.

Table 2.1

Mean correct generate completions for the 3 blocks and 2 lists for experimental and control participants. Standard deviations are in parentheses

\begin{tabular}{|l|l|l|l|l|l|l|}
\hline Lists & \multicolumn{3}{|c|}{ List A } & \multicolumn{3}{c|}{ List B } \\
\hline Blocks & 1 & 2 & 3 & 1 & 2 & 3 \\
\hline Experimental & 15.5 & 15 & 15.12 & 14.12 & 14.62 & 13.87 \\
& $(0.89)$ & $(1.03)$ & $(1.26)$ & $(2.68)$ & $(2.39)$ & $(2.68)$ \\
& 14.37 & 14.62 & 14 & 13.12 & 14.5 & 13.5 \\
& $(1.82)$ & $(1.01)$ & $(0.96)$ & $(2.06)$ & $(2.13)$ & $(2.34)$ \\
\hline
\end{tabular}

\section{Perceptual tests}

\section{Graphemic cued recall}

Table 2.2

Mean encoding condition completions: graphemic cued recall. Standard deviations are in parenthesis.

\begin{tabular}{|l|c|c|c|}
\hline$n=8$ per cell & Baseline & Read & Generate \\
\hline Experimental & 1.5 & 8.5 & 7.25 \\
& $(1.69)$ & $(2.77)$ & $(3.01)$ \\
\hline \multirow{2}{*}{ Control } & 2.0 & 7.25 & 7.37 \\
& $(2.13)$ & $(2.81)$ & $(3.33)$ \\
\hline
\end{tabular}


A 2 (Group: depressed and non-depressed) X 2 (Study Condition: studied and non studied) ANOVA revealed no main effect of Group, ( $p>0.05)$, however there was a significant main effect of Study Condition, $\mathbb{F}(1,14)=$ $73.65, p<0.0001$. This is shown in the better overall performance in the studied conditions $(\bar{X}=7.66, \underline{S D}=2.98)$ when compared to the non studied baseline $(\bar{X}=1.75, \underline{S D}=1.91)$. There was no significant Group $X$ Study Condition interaction, $(p>0.05)$.

A 2 (Group) X 3 (Encoding Condition: read, generate and baseline) ANOVA revealed no significant main effect of Group, $(p>0.05)$ however there was a significant main effect of Encoding Condition, $\underline{E}(2,28)=37.64, \underline{p}<0.001$. Post hoc (scheffé) tests, collapsed across Group showed a significant difference between the baseline $(\bar{X}=1.75, \underline{S D}=1.91)$ and read conditions $(\bar{X}=7.88, \underline{S D}=$ 2.79), $\quad \underline{F}(2,28)=37.45, \underline{p}<0.01$, and a significant difference between the generate $(\bar{X}=7.44, \underline{S D}=3.17)$ and baseline conditions, $\underline{E}(2,28)=30.91, \underline{p}<$ 0.01 . There was no difference between the read and generate conditions, ( $p$ > 0.05). There was no significant Group X Encoding Condition interaction, ( $p>$ $0.05)$.

A 2 (Group) X 2 (Encoding Condition: read and generate) ANOVA performed on studied data corrected for baseline guessing found no significant main effects of Group or Encoding Condition, ( $\underline{p}>0.05)$. Overall performance in the corrected read condition $(\bar{X}=6.06, \underline{S D}=0.88$ ) was not significantly different from those in the corrected generate conditions $(\bar{X}=5.68, \underline{S D}=1.26)$. There was no interaction between Group and Encoding Condition, $(p>0.05)$. 
Word fragment completion

Table 2.3

Mean encoding condition completions: word fragment completion. Standard deviations are in parenthesis.

\begin{tabular}{|l|c|c|c|}
\hline$n=8$ per cell & Baseline & Read & Generate \\
\hline Experimental & $\begin{array}{c}4.62 \\
(2.72)\end{array}$ & $\begin{array}{c}7.37 \\
(1.99)\end{array}$ & $\begin{array}{c}6.37 \\
(3.33)\end{array}$ \\
\hline \multirow{2}{*}{ Control } & $\begin{array}{c}4.62 \\
(2.97)\end{array}$ & $\begin{array}{c}9.62 \\
(3.58)\end{array}$ & $\begin{array}{c}7.37 \\
(2.66)\end{array}$ \\
\hline
\end{tabular}

A 2 (Group: depressed and non-depressed) X 2 (Study Condition: studied and non-studied) ANOVA revealed no significant main effect of Group, $(\underline{p}>0.05)$, however there was a significant main effect of Study Condition, $\underline{F}$ $(1,14)=12.33, \underline{p}<0.05$. This was shown by the better overall performance in the studied conditions $(\bar{X}=7.68, \underline{S D}=2.89)$ when compared to the non studied baseline $(\bar{X}=4.62, \underline{S D}=2.84)$. There was no significant Group $X$ Study Condition interaction, $\underline{\mathrm{F}}(1,14)=.87, \underline{\mathrm{p}}=0.37$.

A 2 (Group) X 3 (Encoding condition: read, generate and baseline) ANOVA revealed no significant main effect of Group, $(p>0.05)$ however there was a significant main effect of Encoding Condition, $\underline{E}(2,28)=10.26, \underline{p}<0.001$. Post hoc (scheffé) tests, collapsed across Group showed a significant difference between the baseline $(\bar{X}=4.62, \underline{S D}=2.84)$ and read conditions $(\bar{X}=8.5, \underline{S D}=$ 2.78), $\underline{E}(2,28)=10.24, \underline{p}<.01$, and a significant difference between the generate $(\bar{X}=6.87, \underline{S D}=2.99)$ and baseline conditions, $\underline{E}(2,28)=3.44, \underline{p}<$ 0.05 . There was no difference between the read and generate conditions ( $p>$ 0.05). There was no significant Group X Encoding Condition interaction, (p $>$ $0.05)$.

A 2 (Group) X 2 (Encoding Condition: read and generate) ANOVA was performed on studied data corrected for baseline guessing found no main effect of Group, ( $p>0.05$ ) but there was a significant main effect of Encoding Condition, $\mathrm{F}(1,14)=5.72, \underline{p}<0.5$. This was reflected in the overall better performance in the corrected read condition $(\bar{X}=3.9)$ compared with the 
corrected generate condition $(\bar{X}=2.25, \underline{S D}=0.15)$. There was no interaction between Group and Encoding Condition, ( $p>0.05$ ).

\section{Conceptual driven tests}

\section{Semantic cued recall}

Table 2.4

Mean encoding condition completions: semantic cued recall. Standard deviations are in parenthesis.

\begin{tabular}{|l|c|c|c|}
\hline$n=8$ per cell & Baseline & Read & Generate \\
\hline Experimental & $\begin{array}{c}1.87 \\
(1.45)\end{array}$ & $\begin{array}{c}5.12 \\
(2.99)\end{array}$ & $\begin{array}{c}7.37 \\
(4.10)\end{array}$ \\
\hline \multirow{2}{*}{ Control } & 2.5 & 6.37 & 10.25 \\
& $(1.77)$ & $(3.58)$ & $(3.05)$ \\
\hline
\end{tabular}

A 2 (Group: depressed and non-depressed) X 2 (Study Condition: studied versus non-studied) ANOVA revealed no significant main effect of Group, $\underline{F}(1,14)=1.73 \underline{p}=0.21$, however there was a significant main effect of Study Condition, $\underline{F}(1,14)=45.46, \underline{p}<0.0001$. This was shown by the better overall performance in the studied conditions $(\bar{X}=7.28, \underline{S D}=3.43)$ when compared to the non studied baseline $(\bar{X}=2.18, \underline{S D}=1.61)$. There was no significant Group X Study Condition interaction, $(p>0.05)$.

A 2 (Group) X 3 (Encoding Condition: read, generate and baseline) ANOVA revealed no significant main effect of Group, $(p<0.05)$ however there was a significant main effect of Encoding Condition, $\underline{F}(2,28)=36.91$, $\underline{p}<0.001$. Post hoc (scheffé) tests, collapsed across Group showed a significant difference between the baseline $(\bar{X}=2.18, \underline{S D}=1.61)$ and read conditions $(\bar{X}=5.75, \underline{S D}=$ $3.28), \underline{E}(2,28)=10.70, \underline{p}<0.01$, and a significant difference between the generate $(\bar{X}=8.81, \underline{S D}=3.57)$ and baseline conditions, $\underline{F}(2,28)=36.93, \underline{p}<$ 0.01. There was also a significant difference between the read and generate conditions, $\underline{E}(2,28)=7.86, \underline{p}<0.01$. There was no significant Group $X$ Encoding Condition interaction, ( $p>0.05$ ). 
A 2 (Group) X 2 (Encoding Condition: read and generate) ANOVA was performed on studied data corrected for baseline guessing. There was no main effect of Group, ( $\underline{p}>0.05)$ but there was a significant main effect of Encoding Condition, $\underline{E}(1,14)=21.79, \underline{p}<0.001$. This was reflected in the overall better performance in the corrected generate condition $(\bar{X}=6.6, \underline{S D}=1.96)$ compared with the corrected read condition $(\bar{X}=3.6, \underline{S D}=1.67)$. There was no interaction between Group and Encoding Condition, $\underline{E}(1,14)=1.5, \underline{p}=0.24$.

\section{Category association}

Table 2.5

Mean encoding condition completions: category association. Standard deviations are in parenthesis.

\begin{tabular}{|l|c|c|c|}
\hline$n=8$ per cell & Baseline & Read & Generate \\
\hline Experimental & $\begin{array}{c}4.37 \\
(2.44)\end{array}$ & $\begin{array}{c}4.62 \\
(1.99)\end{array}$ & $\begin{array}{c}6.62 \\
(1.30)\end{array}$ \\
\hline \multirow{2}{*}{ Control } & $\begin{array}{c}2.62 \\
(1.06)\end{array}$ & $\begin{array}{c}3.87 \\
(2.10)\end{array}$ & $\begin{array}{c}7.0 \\
(3.02)\end{array}$ \\
\hline
\end{tabular}

A 2 (Group: depressed and non-depressed) X 2 (Study Condition: studied versus non-studied) ANOVA revealed no significant main effect of Group, $\underline{F}(1,14)=3.0, p=0.10$ however there was a significant main effect of Study Condition, $\mathrm{E}(1,14)=9.2, \underline{p}<0.5$. This was shown by the better overall performance in the studied conditions $(\bar{X}=5.5, \underline{S D}=2.10)$ when compared to the non studied baseline $(\bar{X}=3.5, \underline{S D}=1.75)$. There was no significant Group $X$ Study Condition interaction, $\mathrm{E}(1,14)=1.4, \underline{p}=0.26$.

A 2 (Group) X 3 (Encoding Condition: read, generate and baseline) ANOVA revealed no significant main effect of Group, $(p>0.05)$ however there was a significant main effect of Encoding Condition, $\mathrm{E}(2,28)=10.31, \underline{p}<0.001$. Post hoc (scheffé) tests, collapsed across Group showed a significant difference between the read $(\bar{X}=4.25, \underline{S D}=2.04)$ and generate conditions $(\bar{X}=6.81, \underline{S D}$ $=2.16), \underline{E}(2,28)=5.60, \underline{p}<.01$, and a significant difference between the 
generate and baseline $(\bar{X}=3.5, \underline{S D}=1.75)$ conditions, $\underline{F}(2,28)=10.96, \underline{p}<$ 0.01 . There was no difference between the read and baseline conditions, $(\underline{p}>$ 0.05). There was no significant Group $X$ Encoding Condition interaction, ( $\underline{p}>$ 0.05).

A 2 (Group) X 2 (Encoding condition: read and generate) ANOVA was performed on studied data corrected for baseline guessing. There was no main effect of Group, $\underline{F}(1,14)=1.4, \underline{p}=0.26$ but there was a significant main effect of Encoding Condition, $\underline{E}(1,14)=11.41, \underline{p}<0.5$. This was reflected in the overall better performance in the corrected generate condition $(\bar{X}=3.3, \underline{S D}=0.41)$ compared with the corrected read condition $(\bar{X}=.75, \underline{S D}=0.29)$. There was no interaction between Group and Encoding Condition, ( $p>0.05)$.

\subsection{Discussion}

Experiment 1 did not find any differences between depressed and control participant performance in the two perceptual tests or between the two conceptually driven tests. Put another way, no differences were found between the tests which were performed under implicit or explicit test instructions. The lack of a deficit in depressed participant performance is in contrast to previous depression implicit - explicit memory experiments. Although these experiments did not manipulate process type at both encoding and test they all found that depressed participant recall was significantly lower on the explicit tests that they used (Danion et al., 1992; Elliot and Greene, 1992).

One possible explanation is that the level of depression experienced by the participants in Experiment 1 (at least as measured by the BDI) was lower than that of other experiments which used students as participants (and much lower than experiments which used clinical participants). For instance Hertel and Hardin (1990) obtained a mean BDI level of 15.29, Hertel and Knoedler (1996) across a series of problem solving tests obtained mean levels between 14.6 and 16.2 (the overall mean was 15.44) and Ruiz-Caballero and Gonzalez (1994) 19.45 for the first experiment and 16.19 for the second. The mean BDI 
level for the current experiment (14.75) while being close to the above, does fall lower than previous experiments in this area. This suggests (without an alternative explanation) that the level of depression experienced by the participants in this study was not of a sufficient depth to induce deficits in conceptual or explicit measures of memory. While no differences were obtained in an implicit test measure, it is possible that if the level of depression was severe enough to produce deficits in an explicit test, deficits in implicit (conceptual) test performance may also be revealed.

A possible alternative explanation for the lack of significant results in this experiment was low power. Due to the design that was used the results from only 8 participants were obtained from each test. While there was no particular trend in the data it remains possible that had Experiment 1 included higher numbers of participants then a significant result could have been found. This suggests that for Experiment 2 a real effort should be made to find a higher number of participants

There was also a problem in the way students were selected for the depressed group. Although a conservative cut off score was used on the initial screen of the BDI (15) this was allowed to fall back to a less conservative measure (nine) on the second screen of the BDI. Previous student depression research has used student samples obtained on the basis of BDI scores of between nine and 12, for example Hertel and Hardin (1990); Ruiz-Caballero and Gonzalez (1994); and Hertel and Milan (1994) however on the rescreen this was not allowed to drop below the level set for the first screen of the BDI. While it was expected that a high cut off score in combination with a rescreen level which had been used as a base level in previous experiments would be sufficient, the level of variation that this criterion allowed could be problematic.

A drop in BDI scores between screenings is a common phenomena, particularly with student participants. Hammen (1980) noted a mean drop of eight points between BDI screenings while Hatzenbuehler, Parpal and Matthews (1983) found $71 \%$ of participants changed classification from 
depressed to non-depressed between BDI screenings (an one week interval was used).

Coyne (1994) has criticised the use of the BDI as the main means of classifying students into depressed and control groups. Coyne regards even elevated scores on the $\mathrm{BDI}$ as representing at best a form of distress rather than depression and that a key feature of this 'state' is its transience. As a result the use of a lower rescreen level on the BDI may pick up students who are experiencing a transient mood state which may not be depression at all. Alternatively, it is possible that participants who were depressed on the first screen of the BDI were somewhat better a week or two later when they performed the experiment and this improved state is reflected in the reduced BDI scores.

Although as predicted no differences were obtained on the explicit perceptual test, caution must be applied before one accepts this as a valid finding. While intact explicit perceptual performance is problematic for the original memory systems view and is supportive of the processing framework, final confirmation of this result must be based on an experiment in which depressive deficits are obtained. It is possible that an experiment using a severely depressed sample may reveal a depressive deficit on such a test. As a result no final conclusion can be reached on which of the processing or memory systems theories provide the most reasonable explanation for depressed participant performance.

Experiment 1 did confirm that most of the tests in the matrix were driven primarily by perceptual or conceptual processes as defined by Blaxton $(1989,1992)$. Both conceptually driven tests were primed significantly more by the generate condition while the word fragment test was primed significantly more by the read encoding condition.

As expected performance in the read condition in both conceptual tests was relatively small, compared to that in the generate condition. As a result this allows little room for the appearance of a depressive deficit in the read condition in a typical conceptual test. Instead this is more likely to appear in the 
generate condition which due to higher priming thresholds allows greater room for between group differences.

The explicit graphemic cued recall test showed no significant difference in priming between the read and generate conditions. While this later finding is in contrast to Blaxton $(1989,1992)$ it is perhaps not surprising to find a significant contribution of conceptual processes in a test performed under explicit or intentional test instructions.

There were a number of additional problems in the design used for Experiment 1. The low power of Experiment 1 has previously been mentioned. This came about since participants completed two out of a possible four tests. While this enabled the experiment to be completed in a reasonable time frame and helped reduce some cross test noise, for each test this resulted in only half the participants contributing data for each test. In order to avoid this in future, it would be best if the data for each test was obtained from every participant taking part in the experiment.

Despite extensive pre-test norming it was found that sometimes a target could be cued by several stimuli. This was particularly the case when two tests, for avamale the catadory acconiatinn toct and the comantir cilad ranall tost 


\subsection{Experiment 2}

While no groups deficits were found in Experiment 1, one explanation for this was the relatively low intensity of the mood state experienced by these participants. A contributing factor towards this was the reduction in BDI scores between the first presentation and that found during the experiment. Also Experiment 1 used relatively few subjects which has resulted in a low level of power.

To reduce the variability in $\mathrm{BDI}$ scores between screenings, a BDI score of 15 was used on the first screen. At test this could not drop below 12 or the participant was removed from the experiment. A slight drop in BDI scores was allowed because it was found in Experiment 1 that virtually all participants dropped by two to three points over a two to three week period. A mean BDI score of 12 was still above the rescreen score of nine used by Hertel and Hardin (1990); Hertel and Milan (1994) and Hertel and Knoedler (1996). By using a more conservative criterion it was hoped to demonstrate the appearance of significant differences in test performance between groups.

Other means were also used to increase the likelihood that a depressive deficit will be found, should this exist. Roediger, Weldon and Challis (1989) noted that the amount of priming in perceptual tests falls off as a function of the dissimilarity between study and test events. They found that typography and modality manipulations all reduced performance, while little or no priming was obtained from encoding manipulations such as studying synonyms, associates or category co-ordinates. They concluded that as the surface features between study and test change, they become increasingly perceptually dissimilar reducing the amount of perceptual priming that can transfer from study to test (see also McDermott \& Roediger, 1994).

Roediger et al. (1989) also noted that in conceptual tests the amount of cue information provided between study and test can be a determinant of test performance. For instance a common finding is that greater priming is obtained on a test of recognition when compared to a free recall test (Tulving \& Watkins, 
1973). This reflects the closer similarity in cue information provided in tests of recognition when compared to free recall, where no cue information is provided. As a result perceptual and conceptual test priming can depend on the amount and type of cue information provided at test. Furthermore priming levels can be reduced if minimum levels of cue information are provided at test.

As a result, for Experiment 2 it was decided to use tests which provided minimal cue information at test. It was hoped that this in combination with a stricter BDI criterion was sufficient to produce the appearance of test/process deficits.

The tests chosen for Experiment 2 were perceptual identification (implicit - perceptual), anagram solution (implicit - mixed) and free recall (explicit conceptually driven). It was also decided that each participant would complete all three tests, in order to increase the power of this experiment compared to Experiment 1. As a result of this decision a explicit - perceptual test was not included in Experiment 2.

Schwartz (1989) used a read/generate encoding manipulation with the perceptual identification test and found that identification thresholds were significantly lower for words read than words generated. This finding supports the categorisation of this test as primarily perceptually driven. Roediger and Blaxton (1987) have also recommended this test as almost fully perceptual. This was mainly due to the fast presentation speeds which allow little time for conscious or conceptual processing. More recently a number of experiments have shown significant effects of a generate encoding manipulation on tests of perceptual identification (see Masson \& MacLeod, 1992; Schwartz, 1989; Toth \& Hunt, 1990). When these claims were more systematically investigated there was evidence that these conceptual effects were largely due to the operation of lexical processes (Weldon, 1991) and possible contamination by explicit/ intentional retrieval processes (Toth, Reingold \& Jacoby, 1994).

Previous experimentation has shown that anagram solution is one of a few implicit tests which draw on approximately equal contribution of perceptual and conceptual processes. For instance Srinivas and Roediger (1990: Expt. 1) 
found no significant difference in priming levels between the no-context (read) and generate conditions for this test. However the degree of perceptual or conceptually driven processes involved in solving anagrams may depend on how close the anagram is to the original word (Srinivas \& Roediger). Others have noted that priming levels also depend on factors such as word length, the number of letter transpositions and word frequency (see Gilholy \& Johnson, 1978; Mendelsohn \& O'brien, 1974; Warren \& Thomson, 1969).

The perceptual identification and anagram solution tests were ideal tests to use to reduce the amount of cue information provided at test. Perruchet and Baveux (1989) noted that the fast presentation times in the perceptual identification test reduce cue information since there is little time for the participant to consciously study the cue. Likewise they note that the anagram test reduces cue information since the random arrangements of the letters bears in many cases little direct resemblance to the original cue.

The free recall test provides no cue information at test. In contrast to the above tests this is an almost purely conceptually driven test, since no data, which could promote perceptual processing is provided at test (Roediger \& Blaxton, 1987). Blaxton (1989) found significant priming from the generate encoding condition while the read and context conditions (where targets were preceded by semantically related items) did not differ. This finding suggests a large conceptual component in the performance of the free recall test. As a result these three tests lie on a continuum between perceptual (perceptual identification), mixed (anagram solution) to strongly conceptually driven (free recall).

For the processing model to be supported depressed participants should show impaired performance on the conceptual explicit test while the perceptual implicit tests should be spared. As previously outlined, previous experiments with depressed participants have found impairments in the explicit conceptual tests that they used (Elliot and Greene, 1992; Danion et al., 1991). This supports the hypothesis that a conceptual deficit should be expected in the free recall test. Furthermore this impairment would be most likely revealed by 
significantly lower depressed participant transfer in the more conceptual 'generate' condition for this test. In contrast the perceptual identification test should be spared, that is no differences should be found in read or generate transfer for this test. This was on the basis that most previous implicit perceptual tests with depressed participants (with the exception of Elliot \& Greene,1992) have not found impairments in depressed participant performance, for example Danion et al. (1991); Denny and Hunt (1992). The appearance of a deficit in the anagram solution test is less certain, although is less likely due to the relatively lower contribution of conceptual processes in this test.

The original memory systems account would also be supported by the finding of intact implicit test and impaired explicit test performance, although this model does not state which processes are likely to be effected in explicit or implicit test performance. Even if the above result were found the consideration of the processes involved through the use of the read - generate encoding manipulation would provide some support for the processing model. That is the advantage of the processing model would be shown if it were found that the conceptually dominant explicit test was impaired (particularly in the more conceptual generate condition) and the perceptually driven implicit tests spared.

\subsection{Method}

\section{Participants and assignment}

\section{$\underline{\text { Criteria }}$}

The same criteria for participation in Experiment 1 was used in Experiment 2. Experimental participants were asked to participate in the experimental session if they scored 15 or above on the BDI, however if the score fell below 12 on the rescreen they were removed from the experiment. 
Control participants were required to score five or less on the BDI on both the first and second screens. None of the participants used in Experiment 1 were used in Experiment 2.

\section{$\underline{\text { Recruitment }}$}

The same procedure used in Experiment 1 was repeated with Experiment 2 except an introductory economics class (700 plus students) was used.

\section{Final assignment}

Twenty experimental participants were used but six participants were removed since their scores dropped below 12 on the rescreen of the BDI. Fourteen matched control participants were tested. The mean age for the experimental group $(\bar{X}=19.71, \underline{S D}=1.68)$ and the control group $(\bar{X}=19.85, \underline{S D}$ $=3.23$ ) were not significantly different, $\underline{t}(26)=10.43, \underline{p}=0.88$. The mean educational level for the experimental group $(\bar{X}=6.60, \underline{S D}=1.07)$ and the control group $(\bar{X}=6.28, \underline{S D}=1.84)$ were not significantly different, $\underline{t}(26)=.56$, $\underline{p}=0.57$.

\section{Memory tests and materials}

All instructions and stimuli were presented on a Silicon IBM clone computer. Stimuli were presented in black against a white screen. A new set of words was used for Experiment 2. All words were checked against a New Zealand written corpus of English to ensure the relevance of the words to New Zealand participants. Words were specifically chosen for a neutral emotional tone, using the same procedure as outlined under Experiment 1.

A final pool of 84 words were chosen after they were normed by undergraduate students. This ensured that the words could be used to create 
anagrams that could be solved correctly at least $30 \%$ of the time. This pool was then split into two lists of 42 words each (list A and list B). All words were five letters long and varied in word frequency between six and 511 occurrences per million. Word length was kept constant to eliminate the effects of word length during the perceptual identification test. Each list contained three blocks of 14 words each, six blocks in total. Statistical analysis confirmed that there were no differences in word frequency between lists or blocks.

Three memory tests were developed, two of which were implicit, perceptual identification and anagram solution and one explicit, free recall. Each implicit test was linked to a specific list, for example the perceptual identification test drew on list $\mathrm{A}$ and the anagram solution test, list $\mathrm{B}$. Since the free recall test did not rely upon the presentation of cues this was not linked to a specific list. The order of presentation of lists and the test associated with each list was counterbalanced across participants.

The three blocks were designed such that during the encoding task was used for the read task, the other for the generate task while the remaining block was held back and used as a baseline. The order of presentation of the three blocks and the associated encoding task/baseline was counterbalanced across participants. The order of presentation of the read and generate encoding tasks was counterbalanced across participants. Generate sentences were normed to $95 \%$ completion using undergraduate students.

During this experiment two encoding sessions were held, one for each of the implicit tests. As a result only words relevant to that test were shown during the encoding test. The first encoding session was held at the beginning of the experiment, the second at the conclusion of the first implicit test. This was done to ensure that the time delay between encoding and test was the same for each of the implicit tests and to rule out any possible confounding effects due to varying time delays between encoding and test. The order of presentation of the words to be read or sentences to be generated was the same across participants. Generate sentences were normed to $95 \%$ correct completion using groups of undergraduate university students. 
To reduce the effects of primacy and recency on the test results the first two words in each read and generate encoding session were always the same. Likewise the last two words were always the same. The same pattern was repeated for the second encoding task. No cues relating to these words were presented during testing. If these words were recalled during free recall they were not included in the results.

The perceptual identification test involved presenting participants with a word at brief exposures which increased until the correct identification was made. The anagram solution task involved participants solving anagrams to form real words. Words to be identified or anagrams to be solved were based on stimuli read, generated or baseline word from the list that the test was based on. Each test began with five 'foil words' (stimuli to be solved which did not appear in list $\mathrm{A}$ or $\mathrm{B}$, these were not scored). This was done to increase the chance that the test would be performed as an implicit test. The explicit task was a free recall test which required participants to write down as many words as they could recall from both encoding sessions.

At test all instructions were provided by the computer. A quasi-random order was used for displaying the read, generated and baseline words, for instance at a maximum not more than two read, generated or baseline words could appear in sequence before the computer displayed a different encoded/baseline word. A different quasi-random order was used for the two implicit tests and across all participants. The computer noted the time (in milliseconds) that each participant took to correctly identify the target during the perceptual identification test. A booklet was provided to the participant during the anagram solution test, this was numbered from 1 - 47. The same font was used throughout the experiment on both the computer and in the task booklets.

Participants also filled in the participant question sheet used in Experiment 1 as well as the BDI. Participants also filled in a participant consent form which included a brief description of the experiment and the participants rights. This described the implicit tests as tests of thinking abilities. 
Participants were tested individually in a quiet private room. After completing the participant question sheet and the ethical approval form, any concerns were dealt with and the experiment began. Participants were asked to sit in front of an IBM computer. A welcome message told participants that they would be asked to take part in a number of tasks, but the nature of these tasks was not specified. This was followed by instructions for the first read or generate task (these are as for Experiment 1).

The same procedure was used for these tasks as used in Experiment 1 except that the pause between the read and generate tasks was reduced to 10 seconds. This was done to reduce the opportunity for any extra covert rehearsal of these words that some participants may choose to engage in. Following the encoding tasks an interpolated task was used (as in Experiment 1).

Following the interpolated task the instructions for the first implicit task were displayed. Instructions were presented by computer and included one example and two practise trials. At the conclusion of the first implicit task the computer informed the participant that there will be a second read - generate task. Participants were told if they push the escape key they could again view the instructions used for the first read - generate task. Otherwise participants were told to push the space bar to begin the session (participants were told whether it was the read or generate task). At the conclusion of the second encoding task an interpolated task required participants to write down as many world capitals that they could think of in 2 minutes. Next the instructions for the second implicit test were displayed; these also included one example and two practise trials. For both implicit tasks the participants wrote down their responses in a numbered task booklet. At the conclusion of the second implicit test the instructions for the free recall test were displayed. At the end of each test the participants were told that they had completed that section and to inform the experimenter of this. 
For the perceptual identification task the instructions stated that the task was to see

"how well you can identify words that are briefly presented on a computer monitor. After you have pressed the space bar you will see $a+$ in the middle of the screen. This will be replaced by a word that will be flashed briefly at you. The word will be replaced by a visual mask $(X X X X X)$. As soon as you have any idea what the word might be please state this aloud. Please respond even if you are uncertain of your answer. If you are incorrect the experimenter will tell you. When you push the space bar the word will be re-presented to you for a longer exposure time. These exposures will build up until a correct identification is made. Each time you wish to see the word please push the space bar and look at the screen at the same time. Once you have correctly identified a word please push the escape key and this will load up the next word for identification. Push the space bar to see this word. Please sit approximately $16 \mathrm{~cm}$ 's away from the screen during this task. Before we start there will be two practise trials. Push the space bar when you are ready to start".

When the participant pushed the space bar a + appeared in the middle of the screen for $800 \mathrm{msec}$. On the first presentation of the word it was presented for $16.7 \mathrm{msec}$ and was then replaced by a visual mask ( a row of six $X^{\prime} x$ ) for 250 msec. Each time the participant was unable to identify the word, or stated the wrong word $16.7 \mathrm{msec}$ was added to the exposures until the correct identification was made. The computer was used to record the results for all participants. A constant level of room illumination was used throughout the experiment for all participants.

The anagram identification task instructions stated,

"this task involves solving anagrams. An anagram is a word that has been mixed up in a random order. By unscrambling the letters a real word can be spelt out, for example zabre is an anagram for zebra. When you push the space bar an anagram will be displayed on screen for 12 seconds. Please solve the anagram by re-ordering the letters into a real word. Please write down the FIRST real word that comes to mind that can be spelt using the letters in the 
anagram. No plural words are used in these anagrams. Each anagram is numbered. After you have solved the anagram please write down the word next to the correct number on the sheet that will be given to you. You do not have to wait for the tone to sound before you right down your answer. If you have not solved the anagram when the tone sounds please move onto the next anagram. To bring up the next anagram please push the space bar. Before we start there will be two practise trials. Push the space bar when you are ready to start".

When the participant pushed the space bar the anagram was displayed on screen for 12 seconds. At the end of that time a tone sounded and the anagram disappeared.

The free recall instructions informed the participant,

"during the session today on two occasions you were asked to read out a list of words or generate words based on sentence cues. Your task is to try and recall as many of these generated and read words as possible. Remember you are trying to recall these words from BOTH occasions. You will be given a blank piece of paper and a pen to do this. You will have four minutes to complete this task".

At the conclusion of the explicit test all participants were left alone and asked to fill in the BDI. This was followed by a debrief. The experimenter sat away from the participant during the test phases but remained in the vicinity of the participant to ensure that all test instructions were being followed.

\subsection{Results}

A t test performed on the BDI scores for the depressed $(\bar{X}=16.50, \underline{S D}=$ 5.2) and the control $(\bar{X}=1.57, \underline{S D}=1.22)$ groups showed that the two groups were significantly different $\underline{\mathrm{t}}(26)=10.43, \underline{\mathrm{p}}<0.001$. The means for correct encoding (generate completions) across the 2 lists for experimental and control participants are shown in Table 3.1. 
A 2 (Group: depressed and non-depressed) X 3 (Block) ANOVA was performed on the correct encoding (generate sentence completions) for list $A$ and showed no significant main effect of Group, ( $p>0.05$ ), no main effect of Block, ( $\underline{p}>0.05)$ and no interaction between Group X Block, $(\underline{p}>0.05)$. A 2 (Group) X 3 (Block) ANOVA on the correct encoding (generate sentence completions) for list B showed no main effect of Group, ( $p>0.05$ ), no main effect of Block, $(p>0.05)$ and no interaction between Group X Block, $(\underline{p}>0.05)$.

A t test was also performed on the interpolated task results and showed no difference between groups for the NZ towns and cities task $(p>0.05)$ and the world capital task $(\underline{p}>0.05)$. $t$ tests were also performed on the order effects for the implicit test, these were found to be non-significant ( $p>0.05)$.

Table 3.1

Mean correct generate completions for the 3 blocks and 2 lists for experimental and control participants. Standard deviations are in parentheses

\begin{tabular}{|l|l|l|l|l|l|l|}
\hline Lists & \multicolumn{3}{|c|}{ List A } & \multicolumn{3}{|c|}{ List B } \\
\hline Blocks & 1 & 2 & 3 & 1 & 2 & 3 \\
\hline Experimental & 14.16 & 13.75 & 14.25 & 14.33 & 14.25 & 12 \\
& $(0.75)$ & $(3.20)$ & $(0.5)$ & $(0.75)$ & $(0.5)$ & $(2.94)$ \\
& 13.5 & 15 & 14.75 & 15.33 & 15 & 14 \\
& $(2.43)$ & $(0)$ & $(0.96)$ & $(0.82)$ & $(1.41)$ & $(2.70)$ \\
\hline
\end{tabular}

Perceptual Identification.

Table 3.2

Mean encoding condition completions: perceptual identification. Standard deviations are in parenthesis.

\begin{tabular}{|l|c|c|c|}
\hline$n=14$ per cell & Baseline & Read & Generate \\
\hline \multirow{2}{*}{ Experimental } & 22.09 & 18.53 & 19.94 \\
& $(5.49)$ & $(2.49)$ & $(4.52)$ \\
\hline \multirow{2}{*}{ Control } & 21.89 & 17.75 & 19.49 \\
& $(4.39)$ & $(1.13)$ & $(3.57)$ \\
\hline
\end{tabular}


A 2 (Group: depressed and non-depressed) X 2 (Study Condition: studied and non studied) ANOVA revealed no significant main effect of Group, $(\underline{F}<1)$ however there was a significant main effect of Study Condition, $\underline{E}(1,26)$ $=14.95, \underline{p}=0.0007$. This was shown by the better overall performance in the studied conditions $(\bar{X}=18.93$ msecs, $\underline{S D}=2.92)$ when compared to the non studied baseline $(\bar{X}=21.64 \mathrm{msec}, \underline{\mathrm{SD}}=4.94)$. There was no significant Group $X$ Study Condition interaction, $(\underline{p}>0.05)$.

A 2 (Group) X 3 (Encoding condition: read, generate and baseline) ANOVA revealed no significant main effect of Group, $(\underline{p}>0.05)$ however there was a significant main effect of Encoding Condition, $\underline{E}(2,52)=10.79, \underline{p}<$ 0.0001 . Post hoc scheffé tests, collapsed across Group showed a significant difference between the baseline $(\bar{X}=21.64, \underline{S D}=4.94)$ and read conditions $(\bar{X}$ $=18.13, \underline{\mathrm{SD}}=1.81), \underline{\mathrm{F}}(2,52)=11.03, \underline{\mathrm{p}}<0.001$. There was no significant difference between the read and generate conditions $(p>0.05)$ or between the generate $(\bar{X}=19.71, \underline{S D}=4.04)$ and baseline conditions $(\underline{p}>0.05)$. There was no significant Group X Encoding Condition interaction, ( $p>0.05)$.

A 2 (Group) X 2 (Encoding condition: read and generate) ANOVA was performed on studied data corrected for baseline guessing. There was no main effect of Group, ( $p>0.05$ ) but there was a significant main effect of Encoding Condition, $\underline{E}(1,26)=5.19, \underline{p}<0.05$. This was reflected in the overall better performance in the corrected read condition $(\bar{X}=3.5)$ compared with the corrected generate condition $(\bar{X}=1.9)$. There was no interaction between Group and Encoding Condition, ( $p>0.05)$.

\section{Anagrams}

Table 3.3

Mean encoding condition completions: anagrams.

Standard deviations are in parenthesis.

\begin{tabular}{|l|c|c|c|}
\hline$n=14$ per cell & Baseline & Read & Generate \\
\hline Experimental & $\begin{array}{c}5.85 \\
(3.18)\end{array}$ & $\begin{array}{c}8.42 \\
(3.03)\end{array}$ & $\begin{array}{c}8.07 \\
(2.43)\end{array}$ \\
\hline Control & $\begin{array}{c}5.78 \\
(2.80)\end{array}$ & $\begin{array}{c}9.00 \\
(2.57)\end{array}$ & $\begin{array}{c}8.78 \\
(2.72)\end{array}$ \\
\hline
\end{tabular}


A 2 (Group: depressed and non-depressed) X 2 (Study Condition: studied and non-studied) ANOVA revealed no significant main effect of Group, $(\underline{F}<1)$ however there was a significant main effect of Study Condition, $E(1,26)=$ 46.98, $\mathrm{p}<0.0001$. This was shown by the better overall performance in the studied conditions $(\bar{X}=8.6, \underline{S D}=2.68)$ when compared to the non studied baseline $(\bar{X}=5.82, \underline{S D}=2.99)$. There was no significant Group $X$ Study Condition interaction, $(\underline{p}>0.05)$.

A 2 (Group) X 3 (Encoding Condition: read, generate and baseline) ANOVA revealed no significant main effect of Group, $(\underline{E}<1)$ however there was a significant main effect of Encoding Condition, $\underline{E}(2,52)=26.07, \underline{p}<0.0001$. Post hoc scheffé tests collapsed across Group showed a significant difference between the baseline $(\bar{X}=5.82, \underline{S D}=2.99)$ and generate conditions $(\bar{X}=8.42$, $\underline{\mathrm{SD}}=2.57), \underline{F}(2,52)=17.78, \underline{p}<0.001$ and between the baseline and read conditions $(\bar{X}=8.71, \underline{S D}=2.8), \underline{F}(2,52)=21.97, \underline{p}<0.001$. There was no significant difference between the read and generate conditions, $(p>0.05)$ nor was there a significant Group X Encoding Condition interaction, $(\underline{p}>0.05)$.

A 2 (Group) X 2 (Study Condition: read and generate) ANOVA was performed on studied data corrected for baseline guessing. There was no main effect of Group, $(\underline{p}>0.05)$ or of Encoding Condition, $\underline{E}(1,26)=.466, \underline{p}=0.50$. This was reflected in similar performances in the corrected generate condition $(\bar{X}=2.6)$ compared with the corrected read condition $(\bar{X}=2.9)$. There was no interaction between Group and Encoding Condition, ( $p>0.05)$.

\section{Free Recall}

Table 3.4

Mean encoding condition completions: free recall. standard deviations are in parenthesis.

\begin{tabular}{|l|c|c|}
\hline$n=14$ per cell & Read & Generate \\
\hline Experimental & $\begin{array}{c}4.28 \\
(2.94)\end{array}$ & $\begin{array}{c}8.14 \\
(2.53)\end{array}$ \\
\hline \multirow{2}{*}{ Control } & 4.78 & 10.71 \\
& $(3.33)$ & $(2.58)$ \\
\hline
\end{tabular}


A 2 (Group: depressed and non-depressed) X 2 (Study Condition: read and generate) ANOVA revealed no significant main effect of Group, $\underline{E}(1,26)=$ $3.71, \underline{p}=0.06$ however there was a significant main effect of Study Condition, $\underline{F}$ $(1,26)=46.98, \underline{p}<0.0001$. This was shown by the better overall performance in the generate conditions, $(\bar{X}=9.42, \underline{\mathrm{SD}}=2.55)$ when compared to the read $(\bar{X}=$ $4.52, \underline{S D}=3.13)$ condition. There was no significant Group $X$ Study Condition interaction, $\underline{E}(1,26)=2.0 \underline{p}=0.17$, (refer Figure 3.1).

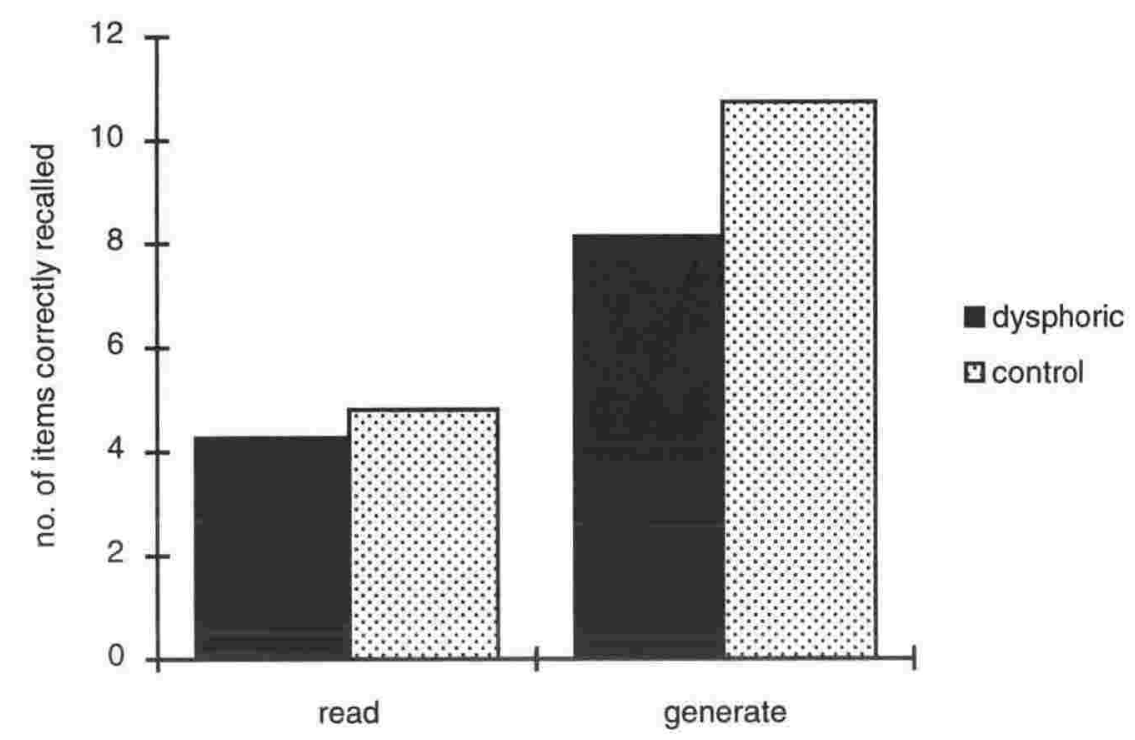

Figure 3.1 Mean correct completions - explicit free recall

\subsection{Discussion}

Experiment 2 found no differences between depressed and control performance in any of the perceptual, mixed or conceptually driven tests. As well, no differences were found between the tests which were performed under implicit or explicit instructions. While the more conservative BDI rescreen criteria produced a higher BDI score this did not result in the appearance of deficits. Similarly, the attempt to reduce the amount of cue information at test along with the associated assumption that this would make the test more difficult to perform did not produce any impairment in depressed participant performance. 
It was expected that performance on the perceptual identification test would not differ between depressed and control participants and also that the anagram solution test was unlikely to differ between groups. According to the processing model such a difference should not occur due to the reasonably high perceptual content in these tests, a process which is thought to be routinely robust in depression. The findings of Experiments 1 and 2 have largely supported the suggestion that these tests are primarily perceptually driven. As a result, two perceptual implicit tests (word fragment completion and perceptual identification) have revealed normal performance in separate experiments (although it cannot be ruled out that these tests could still draw on a specific memory system). While this gives some support to Roediger and McDermotts (1992) assertion that perceptual processes are not disrupted in depressed participants, some caution must be expressed before this is finally accepted.

As previously noted, in experiments where deficits are not found it is difficult to distinguish between the competing explanations of low mood state or whether these tasks are immune to the effects of depression at any mood level. Despite this caution, in previous research perceptual implicit tasks have consistently been intact in experiments using clinical participants (e.g. Danion et al., 1991) and also student participants (Hertel \& Hardin, 1990).

Experiment 2 replicated the finding that the anagram solution test draws approximately equally on perceptual and conceptual processes (Srnivas \& Roediger, 1990) and that free recall draws primarily on conceptual processes (Roediger, 1989). The perceptual identification test collapsed across group revealed a significant difference between the baseline and read conditions, but not between the read and generate conditions.

It was expected that there would be a significant difference between the read and generate conditions in the perceptual identification test (Weldon, 1991). However in Experiment 2 no practise trials were used to calibrate baseline performance (rather the first presentation was set at 16.7 milliseconds which was the fastest presentation the computer could present). It is possible 
that for some participants most of the experiment was performed at above the threshold of awareness and that this could have somehow contributed to an increased conceptual priming effect. Whatever the explanation for this 'conceptual priming' this also illustrates that tests which are thought to mainly draw on one process, under some circumstances can also draw on other processes. It should be noted that some researchers have suggested that priming obtained from a generate encoding manipulation in a perceptual test is not conceptual in nature but instead reflects the operation of lexical processes. (See Fleischman, Gabrieli, Reminger, Rinaldi, Morrell \& Wilson,1995; Weldon, 1991).

While a deficit was expected to occur in the conceptually driven free recall test this was not found. This was despite using equivalent mean BDI levels to those used in previous student based research (Hertel \& Hardin, 1990; Caballero \& Gonzalez, 1994). This null result raises the possibility that the depth and or type of depression experienced by the participants in Experiment 2 was insufficient to induce an impairment in this test (which was both 'explicit' and 'conceptual' in nature). However there was a strong trend in this test, with the group result very close to the level of significance (the interaction was nonsignificant). In other words the depressed group recalled less target words from either encoding condition during the free recall test.

The finding of null results in student - depression research is not a new phenomena. Rathus, Reber, Manza and Kuishner (1994), Experiment 2 found null results using an implicit learning paradigm, using undergraduate students selected on the basis of their BDI scores, (although their BDI mean of 12.8 was relatively low). Rathus et al. also compared depressive performance in the upper and lower deciles of the BDI scores and still found no differences. They concluded that the various levels of depression as measured by the BDI were not associated with differential performance on a 'relatively straightforward' memory task. Rathus et al. did acknowledge that the learning criterion was less demanding in Experiment 2 and that this could be a reasonable explanation for the obtained result. 
Hasher et al. (1985) found no differences in the level or content of depressed and control recall of stories across three experiments (the mean BDI levels were often around 12). The instructions used in these experiments were similar to a free recall (explicit) test, although here participants were asked to recall as much as they could of a previously read story. Subsequent reviews of this experiment (see Ellis, 1985; Isen, 1985; \& Mayer \& Bower,1985) have suggested that the mild mood states and relatively light encoding demands (stories have a logical and organised structure) used in the experiments are reasonable explanations for these results.

What this suggests is that in mild mood states, for instance participants who score between nine and 14 on the BDI, deficits are not routinely found and can depend on the combination of the type of task, test difficulty, for example list length as well as the depth of depression experienced by the participants.

Another explanation for the lack of deficits in Experiments 1 and 2 could be the low power used in these, especially Experiment 1 which only used 8 participants per cell and found an insignificant result. Lack of significant results in this area of research is not new, for instance Hertal and Hardin (1990) tested 11 'naturally depressed' participants, (with a mean BDI of 15.29) and found no significant difference in their explicit test of recognition. Of interest Hertal and Hardin (1990) did record a strong trend in their result, much like Experiment 2. This could suggest that implicit - explicit memory experiments with student participants will never find significant results, yet this is not so (Ruiz and Gonzalez, 1994; also refer to Hertal and Knoedler, 1996, although they used a problem solving methodology). Could it be that in Experiment 2, that if a few more participants had been used a significant result would have been obtained?

Unfortunately very few implicit - explicit experiments using student participants with BDI levels in the 12 - 16 range have been carried out. As a result it is difficult to give a conclusive answer to this question. However Experiments 1 and 2 do suggest that deficits are not mandatory in experiments using low power and similar BDI levels to those used in these experiments. 
Some of the experiments reported above used quite large numbers of participants (Hasher et al., 1985 used 60 odd participants per experiment; Rathus, Reber, Manza \& Kuishner, 1994 a similar number) and a BDI range close to Experiments 1 and 2 and did not find any deficits. In contrast those experiments which have used a higher range on the BDI and lower numbers of participants have always obtained significant deficits (Elliot \& Greene,1992 used 10 participants; Denny \& Hunt, 1992, used 16 participants). This suggests that a primary factor in predicting whether a deficit is likely to occur is the level of the BDI or the presence of a moderate to severe depressive state. In contrast power or numbers of participants is only a secondary consideration in this type of prediction.

To put this another way had Experiments 1 and 2 been repeated with a larger number of participants scoring on a similar range on the BDI to that previously obtained it is possible that a deficit may or may not be obtained. Yet if the BDI level were increased then it would be much more likely that a deficit would be found.

Hertel and Knoedler (1996) have questioned the splitting of university students into depressed or control groups on the basis of BDI scores alone. They suggest that university students who score between nine and 16 on the BDI should be considered dysphoric, while those who score higher might be depressed if they meet certain criteria in a structured clinical interview. They also note that the $\mathrm{BDI}$ is a standard inventory for revealing the syndrome of depression, but not its formal diagnosis. Typically previous student depression memory studies have not made this distinction and have labelled all participants who score over nine as depressed.

On the basis that participants cannot be formally described as depressed without a professional diagnosis, subsequently such participants in the present study will be described as dysphoric.

In sum, while there were no differences in priming between the dysphoric and control participants there was a trend for the dysphoric participants to show reduced conceptual priming in the free recall test. This suggests that under 
conditions where 'severely depressed participants' are used, a conceptual (or explicit) deficit is obtainable, for example Bazin et al. (1994). In previous depression implicit - explicit memory research robust results were found when clinical participants were used. It is to this group of participants that Experiment 3 will turn. 


\subsection{Experiment 3}

Experiments 1 and 2 did not find significant deficits in either the implicit explicit or the perceptual - conceptual test dimensions. While a number of factors could explain this, a reasonable explanation would question the nature and depth of the 'depression' experienced by the university students that were tested.

Where previous experiments have used clinical participants sourced through treatment centres and diagnosed with a depressive disorder the results have largely been clear cut. For instance Elliot and Greene (1992) and Denny and Hunt (1992) found significant deficits in depressed participant performance in their explicit tests, while the former experiment also noted an implicit deficit. In these cases there is far less doubt as to whether the participants used in the experiments were depressed and whether this depressed state was capable of producing depressive deficits.

As a result Experiment 3 will only use participants formally diagnosed with a depressive disorder (major depressive disorder) by a trained professional. It was hoped that with the use of depressed participants that depressive impairments, should these exist will be found in Experiment 3.

Experiment 2 of the present study gave a hint that a deficit could be found in conceptual processes (although this was restricted to a test performed under intentional recall instructions) and it is this factor that will be further explored in Experiment 3. Three tests will be used, the category association test (implicit), the word fragment completion test (implicit) and the free recall test (explicit).

Two conceptual tests (category association and free recall) will be used to further examine the relationship between a memory systems versus processing account in explaining test performance. Should deficits be obtained in both the implicit and explicit conceptual tests this would be problematic for the original memory systems view, while a deficit only in the later would be problematic for the processing model as least as advanced by Blaxton 
$(1989,1992)$. While some theorists have expressed doubt as to whether conceptual deficits would extend to implicit tests (refer Roediger and McDermott, 1992) it is most likely that this would reveal itself in severe conditions such as a diagnosed depression (cf. Blaxton, 1992).

The perceptual word fragment completion test was included to test whether deficits would be extended to perceptual (implicit) processes. Due to timing and design limitations only three tests were included in this experiment. As a result an explicit perceptual test was not included. Experiment 1 had confirmed that the word fragment completion test was primed significantly more by perceptual processes and the category association test by conceptual processes. Likewise Experiment 2 had shown that the free recall test was primed significantly more by conceptual processes.

For the processing model to be supported the depressed participants should show impairments in the conceptual tests, despite the nature of the retrieval instructions in use and normal perceptual implicit test performance. This impairment would most likely be revealed by significantly lower depressed participant transfer in the more conceptual 'generate' condition for the two conceptual tests. In contrast the finding that any impairment was restricted to the explicit or intentional test would favour the multi-memory system hypothesis.

\subsection{Method}

\section{Participants and assignment}

\section{$\underline{\text { Criteria }}$}

All participants had to meet an universal criteria for participation: an age range of 18 to 55, English as a first language, normal or corrected eyesight and a minimum of three years secondary education. Participants also had to have no self reported difficulties in reading and writing. 
The criteria for participation in the experimental group was a diagnosis of a major depressive disorder (according to DSM-IV criteria) by a skilled practitioner, for instance a clinical psychologist. The BDI was only used as a check on the level of depression currently being experienced by the participant at the time of testing. Participants showing symptoms of a bipolar mood disorder were not asked to participate in this study. Although participants exhibiting mild levels of anxiety could be included, participants would be excluded if they were diagnosed with an anxiety disorder. It was acceptable for participants to be diagnosed with a secondary disorder, for example somatoform disorders, adjustment disorders etc.

All participants had to meet a further range of criteria: no psychotic symptoms, no evidence of mental retardation, no major organic symptoms or brain damage and no E.C.T. in the last six months. It was acceptable for the experimental participants to be on a pharmaceutical drug programme.

All control participants had to meet the universal criteria outlined above and were matched to the experimental group for age, sex and education levels. All control participants had to score five or less on the BDI.

\section{$\underline{\text { Recruitment }}$}

Experimental participants were recruited through a variety of inpatient and outpatient treatment units in the Wellington region. Professional staff were given a participant profile sheet which listed the criteria outlined above.

Once a trained professional had made a diagnosis of major depressive disorder, potential participants were informed briefly about the experiment and given a participant consent form to read. Once consent had been obtained the experimenter was contacted and a time made for the experiment to take place.

Control participants were recruited through a variety of community organisations. Potential control participants filled in a participant profile form and the BDI, those who met the criteria outlined above were asked to participate in part two. 
Ten experimental participants were tested and 10 matched controls were also tested. The mean age for the depressed group $(\bar{X}=33.2, \underline{S D}=11.77)$ and the control group $(\bar{X}=34.8, \underline{S D}=10.05)$ was not significantly different, $\underline{E}(1,18)$ $=.107, \underline{p}>0.05$. The mean educational level of the depressed group $(\bar{X}=5.2$, $\underline{\mathrm{SD}}=2.39)$ and the control group $(\bar{X}=6.3,2.62)$ was not significantly different, $\underline{F}$ $(1,18)=.95, \underline{p}>0.05$.

\section{Memory tests and materials}

All instructions and stimuli were presented on an IBM compatible laptop computer. Stimuli were presented in black against a white screen. Target words were obtained from lists used in Experiments 1 and 2 while others were added in order to meet the final criteria outlined below. All words were checked against a New Zealand written corpus of English to ensure the relevance of the words to New Zealand participants. Words were specifically chosen for a neutral emotional tone, checked by a group of community volunteers.

A final pool of 84 words was chosen, this was split into two lists of 42 words each (list $A$ and list $B$ ). All words were between four and nine letters in length and varied in frequency between one and 236 occurrences per million. Each list contained three blocks of 14 words each, six blocks in total. Statistical analysis was used to ensure no difference in word length or frequency between lists or blocks.

Three memory tests were developed, two of which were implicit (word fragment completion, category association) and one explicit, free recall. For each participant the implicit test was linked to a specific list, for example the word fragment completion test drew on list $A$ and the category association test, list $B$. Since the free recall test did not rely upon the presentation of cues this was not linked to a specific list. The order of presentation of lists and the test associated with each list was counterbalanced across participants. 
The three blocks were designed such that during the encoding task one was used for the read task, the other for the generate task while the remaining block was held back and used as a baseline. The order of presentation of the three blocks and the associated encoding task/baseline were counterbalanced across participants as was the order of presentation of the read and generate encoding tasks. Generate sentences were normed to $95 \%$ completion using participants obtained from a variety of community organisations.

During this experiment two encoding sessions were held, one at the beginning of the experiment and the second after the first implicit test. As a result only stimuli relevant for that test were shown during the encoding test. This was done to equate the time delay between encoding and test across both implicit tests. The same order of the read/generate encoding manipulation was used during the two encoding sessions. The order of presentation of the words to be read or sentences to be generated was the same across participants. The same primacy and recency manipulation as used in Experiment 2 was repeated in Experiment 3.

The word fragment completion test involved participants filling in the missing letters of fragments to form complete words, for instance e_e_h_nt (for elephant). The category association test required participants to write down examples of categories, for example four footed animals - deer, pig, cow. In all cases the categories displayed on screen were semantically related to a target word in a particular block. The free recall test required participants to recall any encoded word from either encoding session and write it down. Norming work (using psychology undergraduates) was used to adjust the completion rates of the word fragments and category association targets. Lists of stimuli were presented to participants such as undergraduate students and adults recruited from community organisations who either filled in the word fragments or listed exemplars of categories. Once these were completed correctly $30 \%$ of the time these were included in the final list of stimuli.

At test all instructions were provided by the computer. The same criteria for the quasi-random order used in Experiment 2 were used for Experiment 3 
(different quasi-random orders were used across participants and tests). The first five words of the word fragment completion test were fixed foil words (these were not found in any of the lists) to increase the likelihood that the participant would not become aware of the implicit nature of the test. These words were not scored. In order to equate the timing between the two tests, no foil words were used for the category association test although the first three presented words were always baseline words, these words were scored. For each of the implicit tests a booklet was provided at test. The booklet for the word fragment test was numbered from one to 47 . The booklet for the category completion test was numbered from one to 42 . The same font was used throughout the experiment on both the computer and in the task booklets.

Participants were also required to fill in a participant question sheet; this asked the participants name, age and the number of years of secondary and tertiary education. Participants also indicated their sex, whether English was their first language, whether they had any problems with reading and writing and that they had normal or corrected eyesight. Participants also signed a participant consent form, this included a brief description of the experiment and the participants rights during and after the experiment. Although this stated that the experiment would end with a test of memory (the exact nature of this test was not stipulated) the implicit tests were described as paper and pencil tests of thinking abilities. This was done to try to preserve the implicit nature of these tests. The BDI was used as a check on the level of depression currently being experienced by the participants.

\section{Procedure}

Participants were tested individually. Although the location of testing sometimes varied in all cases a private quiet room was used. The participant sat at a desk with the laptop in front of them. After completing the participant question sheet and an ethical approval form any concerns were dealt with and the experiment began. Participants were asked to sit in front of an IBM 
computer. A welcome message told participants that they would be asked to take part in a number of tasks, but the nature of these tasks was not specified. This was followed by instructions for the first read or generate task (these are as for Experiments 1 and 2). As in Experiment 2 the first two words (primacy) and last two words presented (recency) did not show up in any subsequent tests. There was a 10 second pause between the read and generate tasks. Following the first encoding task an interpolated task required participants to write down as many New Zealand towns and cities that they could think of in 2 minutes.

Following this interpolated task the instructions for the first implicit task were displayed. Instructions were presented by computer and included one example and three practise trials. At the conclusion of the first implicit task the computer informed the participant of a second read - generate task. Participants were told if they pushed the escape key they could again view the instructions used for the first read - generate task. Otherwise participants were told to push the space bar to begin the session (participants were told whether it was the read or generate task). At the conclusion of the second encoding task an interpolated task required participants to write down as many countries of the world that they could think of in two minutes.

Following this the instructions for the second implicit test were displayed; these also included one example and three practise trials. For both implicit tasks the participants wrote down their responses in a numbered task booklet. At the conclusion of the second implicit test the instructions for the free recall test were displayed. At the end of each test the participants were told that they had completed that section and to inform the experimenter of this.

In the word fragment completion test participants were asked to complete the fragments with the first real word that came to mind that could be used. All fragments were presented in lower case. Participants were informed that they would have up to 20 seconds to complete a fragment, once this time was up they would hear a beep. If they had not completed the fragment in that time participants were asked to leave the fragment blank and move onto the next one. To display the next fragment the participant was asked to push the space 
bar. If the participant completed the fragment within the 20 second time frame they could move onto the next fragment by pushing the escape key (which cut out the timer) and then the space bar to bring up the next fragment.

In the category association test participants were informed that,

"this task is to see how well you can list examples of a category. A category is a name for a group of objects or things. For instance the category flower is a label for all objects which are flowers, for example daffodil, geranium etc.".

Participants were told that the computer would display a category name for 20 seconds and that they were asked to write down as many category examples as they could think of during that time. At the end of 20 seconds they would hear a beep; participants were told to stop writing and display the next category name by pushing the space bar.

For both the word fragment completion test and the category association test each stimulus was numbered to the left of the screen. This corresponded to the numbers in the task booklets (participants were informed of this relationship). The free recall instructions were the same as those used in Experiment 2.

At the conclusion of the explicit test the experimental group were left alone and asked to fill in the BDI and place it in an envelope. Control participants were asked to fill in the BDI before the encoding session, both groups of participants were given a debrief at the end of the session. At the conclusion of the experiment all participants were paid $\$ 25$ for their participation. The experimenter sat away from the participant during the test phases but remained in the vicinity of the participant to ensure that all test instructions were being followed. The experiment lasted approximately 55 minutes. 


\subsection{Results}

An ANOVA performed on the BDI scores for the depressed group $(\bar{X}=$ $25.4, \underline{S D}=7.7)$ and the control group $(\bar{X}=2.1, \underline{S D}=1.17)$, revealed that the 2 groups were significantly different, $\underline{F}(1,18)=87.99, \underline{p}<0.001$. t tests performed on the interpolated task results, showed no differences between groups for the NZ cities/ towns task $(\underline{p}>0.05)$ or the countries task $(\underline{p}>0.05)$. Table 4.1 below summarises the means for correct completion during encoding of the generate sentences for the 2 groups for list A and list B. A 2 (Group: depressed and non-depressed) $\times 3$ (Block) ANOVA was performed on the data for list $A$ and for list B. These showed no main effects of Group, ( $p>0.05)$, Block, $(\underline{p}>0.05)$ and no interaction, $(\underline{p}>0.05)$. An ANOVA performed on test order found no differences for either group on the word fragment test $(\underline{p}>0.05)$ or the category association test, $(\underline{\underline{ }}>0.05)$.

\section{Table 4.1}

Mean correct generate completions for the 3 blocks and 2 lists for experimental and control participants. Standard deviations are in parentheses

\begin{tabular}{|l|l|l|l|l|l|l|}
\hline Lists & \multicolumn{3}{|c|}{ List A } & \multicolumn{3}{c|}{ List B } \\
\hline Blocks & 1 & 2 & 3 & 1 & 2 & 3 \\
\hline Experimental & 14 & 13.33 & 14.75 & 14.5 & 15 & 14.33 \\
& $(1.0)$ & $(1.54)$ & $(1.25)$ & $(1.73)$ & $(1.0)$ & $(0.58)$ \\
Control & 14.33 & 13.33 & 15.75 & 15 & 14.66 & 14.33 \\
& $(1.52)$ & $(2.30)$ & $(0.5)$ & $(1.41)$ & $(0.58)$ & $(0.58)$ \\
\hline
\end{tabular}




\section{Word fragment completion}

Table 4.2

Mean encoding condition completions: word fragment completion. Standard deviations are in parenthesis.

\begin{tabular}{|l|c|c|c|}
\hline$n=10$ per cell & Baseline & Read & Generate \\
\hline Experimental & $\begin{array}{c}4.3 \\
(2.0)\end{array}$ & $\begin{array}{c}7.7 \\
(2.94)\end{array}$ & $\begin{array}{c}6.1 \\
(3.17)\end{array}$ \\
\hline Control & $\begin{array}{c}4.6 \\
(1.50)\end{array}$ & $\begin{array}{c}8.7 \\
(2.11)\end{array}$ & $\begin{array}{c}7.0 \\
(2.62)\end{array}$ \\
\hline
\end{tabular}

A 2 (Group: depressed and non-depressed) X 2 (Study Condition: studied and non studied) ANOVA revealed no significant main effect of Group $(\underline{F}<1)$ however there was a significant main effect of Study Condition, $\underline{F}(1,18)=$ $121.86, \underline{p}<0.001$. This was shown by the higher study mean $(\bar{X}=7.37, \underline{S D}=$ 2.71) when compared to the non studied baseline $(\bar{X}=4.45, \underline{S D}=1.75)$. There was no Group X Study Condition interaction, $(p>0.05)$.

A 2 (Group) X 3 (Encoding Condition: read, generate and baseline) ANOVA revealed no significant main effect of Group, $(\underline{p}>0.05)$ however there was a significant main effect of Encoding Condition, $\underline{F}(2,36)=22.99, \underline{p}<0.001$. There was no Group X Study Condition interaction, ( $p>0.05)$. Post hoc (scheffé) tests collapsed across groups showed a significant difference between the read condition $(\bar{X}=8.2, \underline{S D}=2.52)$ and the baseline $(\bar{X}=4.45, \underline{S D}$ $=1.75)$ conditions, $\underline{F}(2,36)=22.90, \underline{p}<0.01$, between the generate $(\bar{X}=6.55$, $\underline{\mathrm{SD}}=2.89)$ and read conditions, $\underline{\mathrm{F}}(2,36)=4.43, \underline{p}<0.05$ and between the generate and baseline conditions, $\underline{F}(2,36)=7.18, \underline{p}<0.05$.

A 2 (Group) X 2 (Encoding Condition: read and generate) ANOVA was performed on studied data corrected for baseline guessing. There was no significant main effect of Group, $(p>0.05)$ however there was a significant main effect of corrected Study Condition, $\underline{E}(1,18)=8.42, \underline{p}<0.01$. This was shown in the better overall performance in the corrected read condition $(\bar{X}=3.75, \underline{S D}=$ 
$0.77)$ compared to the corrected generate condition $(\bar{X}=2.1, \underline{S D}=1.14)$. There was no Group X Study Condition interaction, $(\underline{p}>0.05)$.

Category Association

Table 4.3

Mean encoding condition completions: category association. Standard deviations are in parenthesis.

\begin{tabular}{|l|c|c|c|}
\hline $\mathrm{n}=10$ per cell & Baseline & Read & Generate \\
\hline Experimental & $\begin{array}{c}1.9 \\
(0.87)\end{array}$ & $\begin{array}{c}2.8 \\
(1.31)\end{array}$ & $\begin{array}{c}3.1 \\
(1.96)\end{array}$ \\
\hline Control & $\begin{array}{c}3.2 \\
(1.61)\end{array}$ & $\begin{array}{c}3.6 \\
(2.63)\end{array}$ & $\begin{array}{c}6.3 \\
(1.49)\end{array}$ \\
\hline
\end{tabular}

A 2 (Group: depressed and non-depressed) X 2 (Study Condition: studied and non-studied) ANOVA revealed a significant main effect of Group, $\underline{F}$ $(1,18)=10.51 \underline{p}<0.05$. This was shown by the higher control group mean $(\bar{X}=$ $4.95, \underline{\mathrm{SD}}=2.06)$ when compared to the depressed group mean $(\bar{X}=2.95, \underline{\mathrm{SD}}=$ 1.63). There was a significant main effect of Study Condition, $\underline{F}(1,18)=51.49$, $\underline{p}<0.001$, as shown in the higher studied mean $(\bar{X}=3.95, \underline{S D}=1.84)$ when compared to the baseline mean $(\bar{X}=2.55, \underline{S D}=1.24)$. There was no Group $X$ Study Condition interaction, $\underline{E}(1,18)=3.28, \underline{p}=0.08$.

A 2 (Group) X 3 (Encoding Condition: read, generate and baseline) ANOVA revealed a significant main effect of Group, $\underline{F}(2,36)=10.51, \underline{p}<0.01$. This is shown in the higher control mean $(\bar{X}=4.37, \underline{S D}=1.91)$ when compared to the depressed mean $(\bar{X}=2.6, \underline{S D}=1.38)$. There was a significant main effect of Encoding Condition, $\underline{F}(2,36)=10.50, \underline{p}<0.001$. Post hoc (scheffé) tests, collapsed across groups showed a significant difference between the read $(\bar{X}=3.2, \underline{\mathrm{SD}}=1.97)$ and generate $(\bar{X}=4.7, \underline{\mathrm{SD}}=1.72)$ conditions, $\underline{F}(2,36)$ $=4.86, \underline{p}<0.05$ and between the generate and baseline $(\bar{X}=2.55, \underline{S D}=1.24)$ condition, $\underline{F}(2,36)=9.98, \underline{p}<0.01$. There was no difference between the read and baseline conditions, $(p>0.05)$.

There was a significant Group $X$ Encoding Condition interaction, $\underline{F}(2,36)$ $=3.46, \underline{p}<0.05$. Post hoc (scheffé) tests, between groups showed a significant 
difference between the depressed $(\bar{X}=3.1, \underline{S D}=1.96)$ and control groups $(\bar{X}=$ $6.3, \underline{\mathrm{SD}}=1.49)$ for the generate condition, $\mathrm{E}(2,36)=22.11, \underline{\mathrm{p}}<0.01$. There was no differences for the read condition $(p>0.05)$ or for the baseline condition $(\underline{p}>$ 0.05). This is shown in Figure 4.1.

A 2 (Group) X 2 (Encoding condition: read and generate) ANOVA was performed on studied data corrected for baseline guessing. There was no significant main effect of Group, $(\underline{p}>0.05)$ however there was a significant main effect of corrected Study Condition, $\mathrm{E}(1,18)=10.63, \underline{p}<0.01$. This was shown in the better overall performance in the corrected generate condition $(\bar{X}=2.15$, $\underline{\mathrm{SD}}=0.48)$ compared to the corrected read condition $(\bar{X}=.65, \underline{\mathrm{SD}}=0.73)$. There was also a significant Group $X$ Study Condition interaction, $E(1,18)=$ $6.80, \underline{p}<0.05$. Post hoc (scheffé) tests between corrected groups showed a significant difference between the depressed and control groups for the generate condition, $\underline{E}(1,18)=5.57, \underline{p}<0.05$. There was no difference between corrected groups in the read condition $(p>0.05)$.

\section{Free recall}

Table 4.4

Mean encoding condition completions: free recall.

Standard deviations are in parenthesis.

\begin{tabular}{|l|c|c|}
\hline$n=10$ per cell & Read & Generate \\
\hline Experimental & $\begin{array}{c}2.3 \\
(2.90)\end{array}$ & $\begin{array}{c}1.8 \\
(1.81)\end{array}$ \\
\hline Control & 2.2 & 4.3 \\
& $(1.87)$ & $(1.49)$ \\
\hline
\end{tabular}

A 2 (Group: depressed and non-depressed) X 2 (Encoding Condition: read and generate) ANOVA revealed no significant main effect of Group, $\underline{F}$ $(1,18)=2.89, p>0.05$ and no significant main effect of Encoding Condition, $\underline{F}$ $(1,18)=1.70, \underline{p}>0.05$. There was a significant Group X Encoding Condition interaction, $E(1,18)=4.49, \underline{p}<0.05$, (refer Figure 4.2). Post hoc (scheffé) tests between groups showed better overall recall by the control participants when 
compared for the depressed participants for the generate condition, $E(1,18)=$ $8.31, \underline{p}<0.01$. There was no difference found in the read condition for the depressed and control participants, ( $\underline{p}>0.05)$.

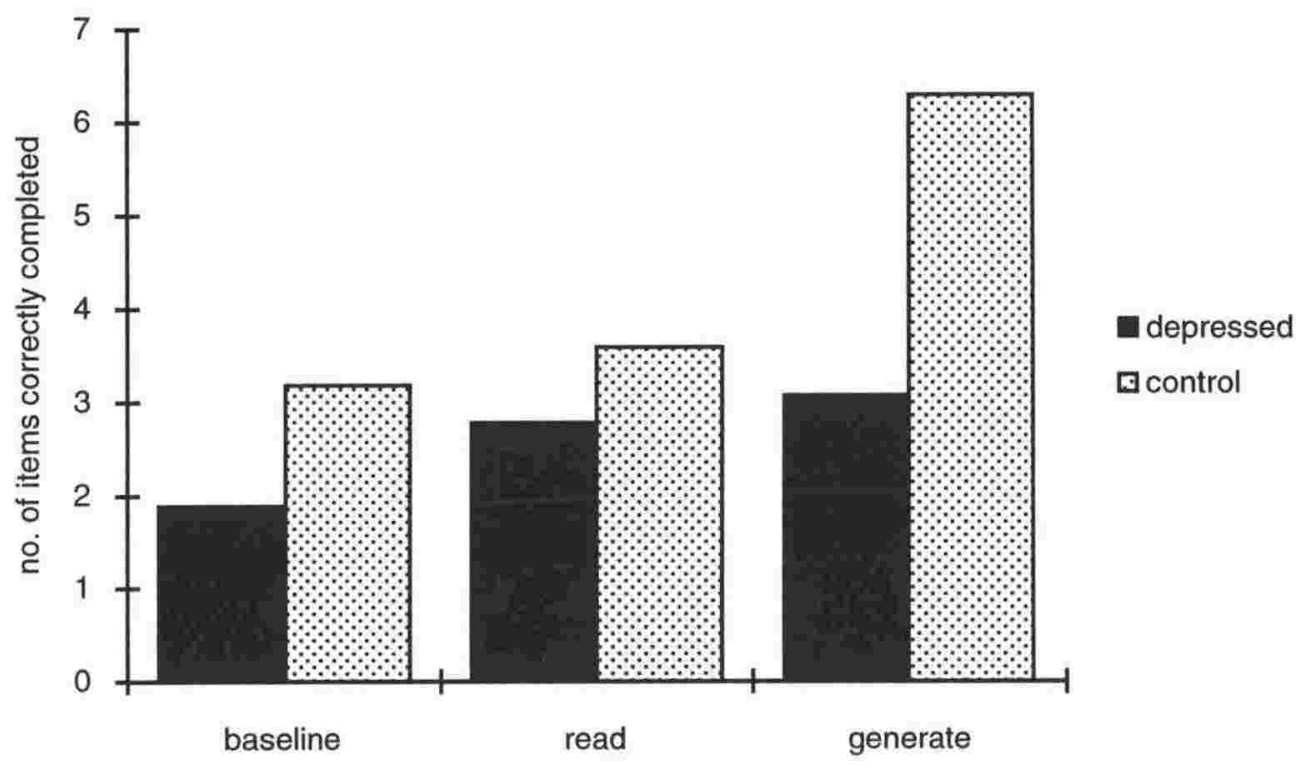

Figure 4.1 Mean correct completions - implicit category association

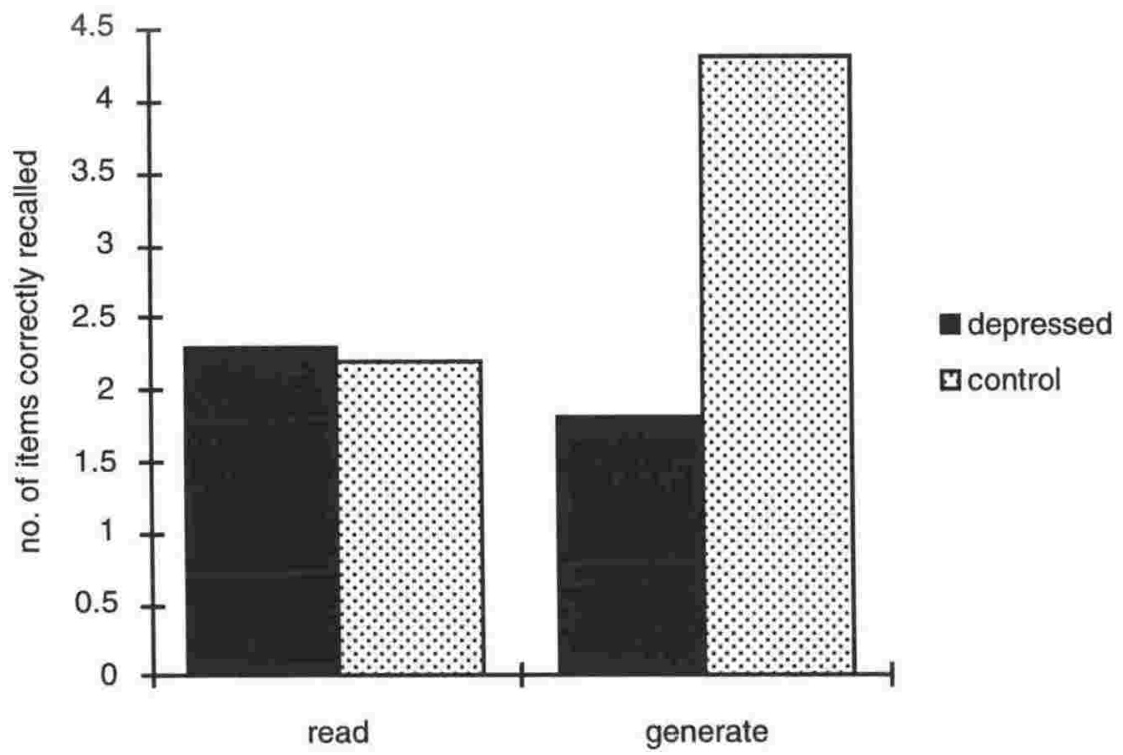

Figure 4.2 Mean correct completions - explicit free recall 


\subsection{Discussion}

In sum, Experiment 3 found significant differences between the depressed and control groups in both the implicit category association and explicit free recall tests. Specifically depressed participants showed significantly less transfer of words from the generate encoding condition, while normal transfer was obtained in the read condition. No differences were found in the implicit perceptual test.

The most important finding of Experiment 3 was that the depressed participants showed deficits in transfer in both the implicit and explicit 'conceptual' tests, although these same participants showed normal patterns of performance in the implicit perceptual test. This finding is similar to those of Blaxton (1992) who has described an impairment in generate transfer within a conceptual test as a 'conceptual deficit'; although it should be noted that her findings were not based on the use of depressed or dysphoric participants. Furthermore the results of Experiment 3 also validates proponents of the processing model who have stated that the test dissociations commonly found in 'traditional' implicit - explicit test performance are best explained with reference to the confounded design used in past depression memory research (refer Table 1.1). That is deficits are typically obtained in explicit tests because they usually tap conceptual processes, which are impaired in depressed participants. Likewise normal performance is found with implicit tests because they usually tap perceptual processes which are not subject to interference in depressed participants.

The finding that the conceptual deficit also extended to the implicit conceptual test is best accommodated by a processing account of memory. In Experiment 3 the dissociations were between impaired conceptual and unimpaired perceptual processing and not between the implicit and explicit versions of these tests. These dissociations add to the predictive power of the processing account and adds another participant group, that is depressed participants for which the theory has successfully explained test dissociations. 
These results also suggest that implicit memory tests cannot be viewed as all behaving in the same way across a range of test situations. As predicted by the processing framework, tests can behave according to their predominate form of processing with implicit perceptual tests sometimes behaving in opposition to implicit conceptual tests.

These findings are problematic for the original memory systems model. This model would need to explain why a consideration of process type was a better predictor for the dissociations obtained than a consideration of a variety of memory systems. The memory systems model does not predict impairments in implicit processes, yet Experiment 3 found this impairment in a conceptual implicit test. For the memory systems model to explain this impairment it will need to assume that under some circumstances this type of test can be impaired. Furthermore this model would need to specify which system is disrupted by a depressed state and the mechanism by which this occurs.

While Experiment 3 found deficits in conceptual transfer, Experiments 1 and 2 did not. While there were some differences in the tests used across these experiments, the major difference was in the type of participant tested. Experiments 1 and 2 used dysphoric or possibly mildly depressed university students while Experiment 3 used participants who had a diagnosis of a major depressive disorder. This suggests that these differences in performance are best explained with reference to the nature and depth of depression experienced by the different participant groups. Furthermore conceptual deficits will only appear in participants who are experiencing moderate to severe forms of depression, while deficits will not be found in the dysphoric state characteristic of the participants used in Experiments 1 and 2. Progress on this issue will not be made until there is further research on the exact nature of the dysphoric mood states experienced by students who are selected via a self report inventory such as the $\mathrm{BDI}$.

The results of Experiment 3 also lend weight to the earlier suggestion that it is the BDI level or presence of clinical depression that is a primary factor in determining whether a deficit will occur and the number of participants used 
a secondary factor. For instance in the case of Experiment 3 less participants were used than Experiment 2 yet robust deficits were found in the later.

Experiment 3 did not find any evidence of a deficit in the perceptual word fragment completion test. This was despite the severe depression experienced by the participants and suggests that perceptual processes are not disrupted, even in severe forms of depression. Such a finding suggests that the results for the implicit perceptual tests in Experiments 1 and 2 would be unlikely to change, even if moderate to severely depressed participants had been used in these experiments. The question as to whether this can be extended to an explicit perceptual test, for instance the graphemic cued recall test can only be answered by using this test with participants experiencing a severe depression.

Experiment 3 has again shown the utility of using an encoding manipulation to confirm the conceptual or perceptual status of the tests that were used. This found that the word fragment completion test was primed significantly more by the read encoding condition. The category association test was primed significantly more by the generate encoding condition. The free recall test was primed significantly more by the generate encoding condition, although this was only found with the control participants.

This manipulation had the added advantage of showing the differences and similarities between depressed and control participant performance. For instance in both conceptual tests control participants showed a generate encoding advantage over read while both control and depressed participants showed a read advantage in the perceptual test. These results do not differ from those that were found in Experiments 1 and 2, suggesting that this is a robust finding.

There was an interesting finding in the free recall test that was used in Experiment 3. Depressed participants recalled less generate words than read words (this difference was not significant) which is unusual for free recall test performance which usually favours semantic encoding (see Jacoby, 1983). While this illustrates the effect of a conceptual deficit, in general both control 
and depressed performance was very low in this test, compared to the other tests. Despite the robust priming found in the free recall test in Experiment 2, in this case a floor effect appears to have occurred. Low levels of recall in explicit tests using participants obtained from special populations can be reasonably common. Light and Singh (1987: Expt. 1) found a floor effect in their free recall tests using young versus old participants. Burke and Light (1981) have also commented that floor effects are a common problem in studies manipulating age and orienting task. Although this was not necessarily an unusual finding this does raise the question of task difficulty and the extent to which tests which differ in task difficulty can be compared.

Cermak et al. (1995) have criticised Blaxton $(1989,1992)$ for relying on four totally different paradigms in the test design that she used. They claim this results in a confound between test difficulty and the manipulation of conceptual and perceptual processes. For instance a finding of an impaired free recall test and a normal word fragment completion test could be attributed to the different processes involved or to differences in the amount of cue information provided at test. While Experiments 1 to 2 have not equated test difficulty, previous implicit - explicit memory depression research (see Table 1.1) have also not done this.

The retrieval intentionality criterion (Bowers \& Schacter, 1990; Schacter et al., 1989) is one means of equating task difficulty across tests. The retrieval intentionality criterion in operation holds all conditions at study and testing constant by using the same cues across these conditions. All that varies are the instructions used. For the criterion to be established a dissociation is required between the implicit and explicit forms of the test. The logic states that if intentional retrieval processes are used during the implicit test, no dissociation will be obtained between the implicit and explicit tests. That is both tests will be performed as if they are explicit tests leading to a similar pattern of performance.

The retrieval intentionality criterion is important in another sense. There is a potential confound in explaining a deficit in the implicit conceptual test. 
Without a specific check on explicit contamination within this test it is difficult to conclusively state whether a deficit has occurred due to a conceptual deficit or due to explicit contamination (or perhaps a mixture of the two). For instance Rappold and Hashtroudi (1991) used the retrieval intentionality criterion to show that there was no explicit contamination in their implicit test of category production. There were no effective means of checking for possible contamination in Experiments $1-3$, even though this could have occurred. However it is unlikely that this contamination would have occurred straight away in that typically participants do not realise the nature of an implicit test, if at all until a reasonable number of cues have been completed (see Schacter et al., 1989). Such test awareness is also less likely to occur in participants presumably low in motivation and test awareness (Hertel \& Hardin, 1990). As a result while there is some doubt in concluding that an implicit conceptual deficit did occur this doubt is relatively minimal.

The retrieval intentionality criterion is not necessarily without its own set of problems. Toth, Reingold and Jacoby (1994) have criticised the criterion for not taking into account potential differences in sensitivity or response criteria across the 2 tests. They also claim that it does not take into account the potential impact of automatic processes on direct tests of memory. As a result a direct test may not be the best benchmark against which to compare performance on an indirect test. However the use of the same cues should help equate test difficulty in as far as the same cue information is provided at test, which is an improvement over Experiments 1 - 3.

There are a number of other methods available to establish independence between tests, for example the method of stochastic independence (see Hayman \& Tulving, 1989) and the process dissociation procedure (Jacoby, 1991; Toth, Lindsay \& Jacoby, 1992). The process dissociation procedure has the advantage of separating out the relative contributions of automatic and controlled processes within task performance and is not effected by differences in task sensitivity between tests (Jacoby, 1991). This will be expanded on in a later section. A 'problem' with the process 
dissociation procedure is that it requires participants to be split into two groups (inclusion and exclusion conditions), effectively reducing the power of the test.

A further problem with Experiment 3 is a relative lack of power. This reflects the relatively low numbers of diagnosed participants that were obtained for this experiment. In contrast student participants are an easier group to access and tend to be available in higher numbers (Ruiz-Caballero \& Gonzalez, 1994, tested 20 participants). While null results were obtained using student participants (i.e. Experiments 1 and 2), several previous experiments have obtained significant results from this source (Hertel \& Knoedler, 1996; Ruiz-Caballero \& Gonzalez, 1994). Experiments 1 and 2 suggest that when student participants are used, high inventory cut off scores combined with multicriteria (e.g. the use of several inventories) should be used to help reduce the variability that these participants can exhibit. Experiment 4 will use participants sourced through a student population and will use a design that does not violate the retrieval intentionality criterion. 


\subsection{Experiment 4}

Experiment 3 found conceptual deficits in both an implicit and an explicit test, using participants sourced through a clinical population. Experiments 1 and 2 did not find any evidence of conceptual deficits, using participants sourced through a student population. However Experiments 1 - 3 violated the assumptions of the retrieval intentionality criterion. Since the criterion has been violated this can make comparisons between tests, even within the same experiment problematic.

More recently, experimental designs have emerged which do not violate this criterion. For instance Cermak et al. (1995) redesigned Blaxtons $(1989,1992)$ four way matrix in order to meet the requirements of the criterion.

Cermak et al. (Experiment 1B) used a semantic cue for an implicit semantic production task (write down a list of the first six words with a similar meaning that comes to mind) and the same cues for an explicit semantic cued recall task (use the cue to recall six words from the study list with a similar meaning). As a result cues were held constant across tasks and only the instructions were varied during the experiment. Cermak et al. also used the read - generate encoding manipulation and found significant priming from the generate condition in both tests (see Experiment 2B) confirming that these tests were conceptually driven. The same design was used in Experiments 1B and 2B, using graphemic cues in order to manipulate the operation of perceptual processes.

A similar design to that used by Cermak et al. (1995) was used for Experiment 4. The tests that were used followed the same logic as above and are commonly described as the word associate (implicit) and word associate cued recall (explicit) tests (see Vaidya et al.,1995). During the former, the participant is asked to use the cue word to think of the first semantically related word that came to mind and write it down. During the later test the participant is asked to use the cue word to recall a semantically related word from the study list. 
Due to time limitations the test combination in Experiment 4 was limited to the manipulation of conceptual implicit and explicit tests. This had the added advantage of seeing whether conceptual deficits could be found in both implicit and explicit tests, using student participants in a test where the retrieval intentionality criterion was not violated.

There is a potential problem in comparing the same conceptual cues within implicit and explicit test versions (Roediger \& McDermott,1993). Roediger (1990 cited in Roediger \& McDermott,1993) has suggested that similar factors, for example categorisation and organisation are at work when participants are engaged in explicit or implicit tests using the same conceptual cues. If this were the case parallel patterns of performance could occur which would give the appearance that the retrieval intentionality criterion has been violated. Yet parallel effects on two tests do not indicate that both tests are tapping intentional retrieval. Instead no strong claim can be made about whether the same or different retrieval processes have been used (Roediger \& McDermott,1993). A number of researchers have found different patterns of retrieval using the same conceptual cues on explicit - implicit versions of a test, see Cermak et al. (1995); Rappold and Hashtroudi, (1991). As a result it should be possible to obtain different patterns of performance between these two tests, even if deficits are found in both tests.

The processing model would predict that depressive impairments should appear in both the implicit and explicit versions of these tests. However it is possible that by using dysphoric participants sourced through a student population these participants will exhibit a different pattern of performance to those found in Experiment 3. For instance it could be that in dysphoria, a implicit conceptual deficit is not found (see Roediger \& McDermott, 1992).

In a major review of implicit memory Roediger and McDermott (1993) have suggested a number of guidelines to help reduce explicit contamination and other 'noise' issues especially when using implicit tests. These include the use of large sets of stimuli so that even if participants try to use intentional retrieval strategies, these will be relatively difficult to use. 
They recommend keeping the proportion of studied words (as opposed to baseline words) in a test below $50 \%$, for the same reason. They also recommend the use of several interpolated tasks to increase the chance that the implicit test was viewed as just one more in a series of tasks and beginning the implicit test with multiple filler 'cues' before the first study cue.

While a number of these suggestions have already been included in Experiments $1-3$, for example the use of filler items and interpolated tasks, a number of changes were made to Experiment 4 to take into account these other suggestions. Baseline items were expanded to match the number of stimuli used in both the read and generate conditions and three interpolated tasks were used prior to the implicit test.

While Experiment 4 was designed to provide as pure a measure as possible of both implicit and explicit test processes, there is still the 'problem' of using student participants. Unless changes were made to the selection criteria used in Experiment 2 there was a real possibility of again obtaining a null result.

In a major review of student versus clinical depression, Vrendenburg, Flett and Krames (1993) recommended the use of multiple criteria, for example several self report measures and high cut off scores as a means of increasing the likelihood that a 'depressed' sample will be obtained. On this basis a tighter inventory criteria was used for Experiment 4. That is a BDI score of 16 on the initial screen, this could not drop below 14 on the rescreen. A secondary measure was also included, the Centre for Epidemiological Studies Depression scale [CES-D], (Radloff, 1977). Participants were also required to meet a conservative criteria for this scale (rescreen score of 14) otherwise they were removed from the experiment.

The CES-D scale measures a range of symptomatology that is both similar and different from the BDI (Radloff, 1977). Radloff has shown the CES-D to have very high internal consistency, good construct validity and adequate test-retest reliability. It was predicted that by combining the criteria from both scales and using conservative cut off scores this would increase the likelihood 
that a genuine dysphoric sample was obtained. Furthermore it was hoped that this sample would exhibit many of the features of actual depression including memory impairments.

Hertel and Knoedler (1996) also recommended the use of a questionnaire to rule out participants who score highly on the BDI due to factors such as sickness, alcohol or drug dependency disorders. In the light of this recommendation the participant profile form was expanded to include questions on sickness and alcohol use.

Experiment 3 showed the utility of using the processing model with depressed participants. As a result, for Experiment 4 the processing model predicted that dysphoric participants should reveal impairments in both conceptual tests despite the nature of the retrieval instructions in use. It was expected that this impairment would be revealed by significantly lower depressed participant transfer in the more conceptual 'generate' condition for these tests. While there was some doubt as to whether this result would be repeated using dysphoric participants, it would be problematic for the processing model if this result were not found. Instead the original memory systems model would be supported if it were found that any impairment was restricted to the test using intentional (explicit) retrieval instructions.

\subsection{Method}

\section{Participants and assignment}

\section{$\underline{\text { Criteria }}$}

The same 'universal' criteria as used in Experiments 1 and 2 was used for this experiment. The criteria for contacting participants to participate was, for the experimental group, a BDI score of 16 on the first screen, if the rescreen fell below 14 participants were removed from the experiment. A further criteria was a score of 14 or above on the CES-D for experimental participants. The 
CES-D was only used at the time of test, it was not used as part of the initial screen of participants (due to timing difficulties). Control participants had to score five or less on the CES-D. Control participants were asked to participate if their BDI scores were five or under on the first and second screen.

Participants were also asked questions on whether they were taking prescribed medications, whether they were under medical care and the amount of alcohol they regularly consumed. If participants reported symptoms that could indicate a moderate to serious medical illness or a substance abuse disorder they were removed from the experiment.

\section{$\underline{\text { Recruitment }}$}

The BDI was passed around introductory quantitative and calculus studies labs. In all approximately 400 students were sampled. It was emphasized that students were not required to fill in the BDI and that participation in part two was voluntary. After scoring the BDI's the class was split into three groups according to the BDI score criteria outlined above.

Approximately five to seven days after completing the BDI, students who scored over 16 were contacted and asked to participate in part two. Once a number of experimental participants had been tested those who had scored under five were contacted and asked to take part in part two. The control group were matched for age, sex and education levels.

\section{Final assignment}

Eighteen experimental participants were tested, however four participants were removed, three because their BDI score dropped below fourteen on the second screen and one due to difficulties in the use of English. As a result fourteen experimental participants were included for analysis and fourteen matched control participants were also tested. The mean age for the dysphoric group $(\bar{X}=21.92, \underline{S D}=4.29)$ and the control group $(\bar{X}=20.21, \underline{S D}=$ 
2.39 ) were not significantly different, $\underline{E}(1,26)=1.707, \underline{p}>0.05$. The mean educational level for the dysphoric group $(\bar{X}=6.15, \underline{S D}=1.16)$ and the control group $(\bar{X}=6.78, \underline{S D}=1.18)$ were also not significantly different, $\mathrm{E}(1,26)=1.96$, p $>0.05$.

\section{Memory tests and materials}

All instructions and stimuli were presented on an IBM computer. Stimuli were presented in black against a white screen. Target words were obtained from lists used in Experiments 1,2 and 3 and a number of further words were added to meet the final criteria outlined below. All words were checked against a NZ written corpus of English to ensure they were relevant for NZ participants and all words were selected for a neutral emotional tone (using the same criteria as outlined in Experiment 1).

A final pool of 104 words was chosen, which was split into two lists (list A and list B) of 52 words each. All words were between four and 10 letters in length and varied in frequency from one to 511 occurrences per million. Furthermore each list contained four blocks of 13 words each, eight blocks in total. Statistical analysis was used to ensure no difference in word length or frequency between lists or blocks.

Two memory tests were developed, the implicit word association test and the explicit cued word association test. In the former the participant was required to write down the first semantically related word that came to mind and in the explicit version the participant had to use the cue to think back and recall a semantically related target from the encoding session. The explicit test always followed the implicit task.

During encoding, each test used two blocks from one list while two blocks from the other list were used as baseline blocks (this doubled the number of baseline words used in Experiments 1 - 3). For each test, one block was used for read targets while the other block was used for generate sentence targets. The order of presentation of the two lists and four blocks and the 
associated encoding/ baseline manipulations were counterbalanced across participants. The order of presentation of the read and generate encoding tasks was counterbalanced across participants.

There was only one encoding session used during this experiment. During encoding the read and generate blocks associated with the implicit test were always shown first. The order of presentation of words to be read or generated was the same across participants. Generate sentences were normed to $95 \%$ completion using groups of university undergraduate students. The same primacy and recency manipulations as used in Experiments 2 and 3 were used in this experiment.

Following the encoding phase three interpolated tasks were presented by the computer to the participants. The interpolated tasks were the NZ towns and cities task (as used in Experiments 1 - 3), the Japanese tourist task and the doubled height task. These later tasks asked the participants to write down as many ways to increase the number of Japanese tourists visiting NZ and to list the consequences if everyone doubled in height over night. For all three tasks a time limit of two minutes was given; the order that the tasks were presented was counterbalanced across participants.

During the test phase, cues that were semantically related to the target word were presented on a computer. The lists were designed so that only one cue was related to a target word. Norming work (using university students) was used to adjust the completion rates of the cues. Lists of stimuli were presented to participants who wrote down two exemplars of that category. Once these were completed correctly $30 \%$ of the time they were included in the final list of stimuli.

A quasi-random order was used for displaying the read, generate and baseline words at test. No more than two generate or read words could appear in a row, while no more than three baseline words could appear in a row. A different quasi-random order was used for each test and across all participants. During the explicit test, participants were told that some presented cues would not relate to words that were read or generated (i.e. the baseline words). They 
were to write down a semantically related word or leave these blank. For each of the tests a answer booklet was provided to the participants. These consisted of a number of stapled pages, numbered from 1 to 52 . The same font was used throughout the experiment on both the computer and in the answer booklets.

Participants were also asked to fill in a participant question sheet. As well as the standard questions asked in Experiments 1 - 3 some additional questions were asked. Participants were asked to list any prescribed medications they were taking, if they were under a doctors care they were asked to list the type of problems this was for. They were also asked to list the type, amount and frequency of alcohol they had recently consumed. A participant consent form was also designed as part of the ethical approval procedure, this included a brief description of the experiment and the participants rights.

The BDI was used as a check on the level of depression experienced by participants. A secondary measure the CES-D was also used. Radloff (1977) has shown that this measure has good reliability and validity in a number of general population samples.

\section{Procedure}

Participants were tested individually in a quiet private room. After completing the participant question sheet and the ethical approval form, any concerns were dealt with and the experiment began. Participants were asked to sit in front of an IBM computer. A welcome message told the participants that they would be asked to take part in a number of tasks, but the nature of these tasks was not specified. This was followed by the instructions for the read or generate tasks (as for Experiments 1 - 3). Immediately following the read and generate encoding session the instructions for the interpolated tasks were displayed. Participants were told when they were ready to start writing to push the space bar, after two minutes a beep would sound and the instructions for 
the next interpolated task was displayed. Participants were told to stop writing when the beep sounded and to start reading the instructions for the next task.

After all three interpolated tasks had been completed the instructions for the implicit task were given. These stated,

"Shortly you will see a series of words displayed on the screen one at a time. You are asked to write down the first word that comes to mind when you see each word. This word should have some meaningful relationship to the word on the screen. For instance if you see the word flower the first word that might come to mind is daffodil". Participants were told that they would have 15 seconds to think of an answer, at the end of that time they would hear a beep. If they had not thought of an answer at the end of that time they were told to move on to the next word, by pushing the space bar. If they completed the word before the 15 seconds was up they were told to push the space bar to display the next word; this also reset the timer. Participants were also told if they were not clear about these instructions to tell the experimenter and he would go through them. At the conclusion of the implicit test a message stated,

"this part of the experiment is complete. Please tell the experimenter".

Following this the explicit test instructions were displayed. These stated,

"you are now asked to take part in a test of memory. At the start of the session today you were asked to read out a list of words and to guess a list of words based on a sentence cue. Your task today is to try and recall both sets of words. To help you do this you will be shown a list of cue words. Each cue word will have a similar meaning to the words you are trying to recall. For instance if you read the word chair at the start of the session today the cue word you might be shown is table. However about half of the cue words have no relationship to the words you read or generated at the start of the session today. If the cue word does not remind you of a word you read or generated earlier, feel free to move on or take a guess as to what an associated word might be". The instructions for timing as given in the implicit test were repeated. Participants were told to clarify any misunderstandings with the experimenter before 
starting the experiment. At the conclusion of the explicit test a message stated " the experimental session is now complete. Please tell the experimenter".

During both tests cues were numbered to the left of the screen $(1-52)$. Participants were given an answer booklet, numbered 1 - 52 to write down their answers. The instructions told the participants to write down their answer next to the number in the booklet that corresponded to the number next to the test cue on the screen. The booklet began with three pages headed A, B and $\mathrm{C}$. Each page was used for recording the answers for an interpolated task.

At the conclusion of the explicit test the experimental group were left alone and asked to fill in the BDI and CES-D and place these in an envelope. Control participants were asked to fill in the BDI and CES-D before the encoding session, both groups of participants were given a debrief at the end of the session. At the conclusion of the experiment participants were paid $\$ 10$ for their participation. The experimenter sat away from the participant during the test phases but remained in the vicinity of the participant to ensure that all test instructions were being followed. The experiment lasted 45 - 50 minutes.

\subsection{Results}

An ANOVA performed on the BDI scores for the dysphoric $(\bar{X}=20.64$, $\underline{\mathrm{SD}}=6.13)$ and the control group $(\bar{X}=2.36, \underline{S D}=1.78)$ showed that the 2 groups were significantly different $\underline{E}(1,26)=114.72 \underline{p}<0.001$. Similar analysis was performed on the CES-D scores for the dysphoric $(\bar{X}=21.43, \underline{S D}=6.48)$ and the control groups $(\bar{X}=0.21, \underline{S D}=0.8)$ showed that the 2 groups were significantly different, $\underline{\mathrm{F}}(1,26)=79.59, \underline{\mathrm{p}}<0.001$.

$\mathrm{t}$ tests were performed on the interpolated task results. This showed no difference between groups for the cities $(\underline{p}>0.5)$ and the Japanese tourist problem $(p>0.5)$ however there was a significant difference for the doubled height problem, $\underline{\mathrm{t}}(26)=2.04, \underline{\mathrm{p}}<0.05$. This is shown in the slightly better overall performance by the dysphoric group $(\bar{X}=7.07, \underline{S D}=1.9)$ compared to the control group $(\bar{X}=5.86, \underline{\mathrm{SD}}=1.17)$. 
Table 5.1 below summarises the means for correct encoding (generate sentences) for the 2 groups for list A and list B . A 2 (Group: dysphoric and control) $\times 4$ (Block) ANOVA was performed on the data for list A and showed no significant main effect of Group, $(\underline{p}>0.05)$ although there was a significant main effect of Block, $\underline{F}(3,18)=13.03, \underline{p}<0.01$. There was no significant Group $X$ Block interaction ( $p>0.05$ ). A 2 (Group) $\times 4$ (Block) ANOVA was performed on the data for list B and showed no significant main effect of Group, ( $p>0.05)$, no significant differences between Blocks $(\underline{p}>0.05)$ and no significant Group X Block interaction, ( $\underline{p}>0.05)$.

Table 5.1

Mean correct generate completions for the 4 blocks and 2 lists for experimental and control participants. Standard deviations are in parentheses

\begin{tabular}{|l|l|l|l|l|l|l|l|l|}
\hline Lists & \multicolumn{5}{|c|}{ List A } & \multicolumn{5}{|c|}{ List B } \\
\hline Blocks & 1 & 2 & 3 & 4 & 1 & 2 & 3 & 4 \\
\hline Experim & 13.25 & 13.25 & 14 & 16 & 15.33 & 15 & 14 & 15 \\
ental & $(0.5)$ & $(0.5)$ & $(1.41)$ & $(0)$ & $(0.58)$ & $(1.0)$ & $(1.0)$ & $(1.0)$ \\
Control & 14.5 & 12 & 14.25 & 15.25 & 15.33 & 15 & 15 & 16 \\
& $(1.73)$ & $(1.82)$ & $(1.5)$ & $(0.5)$ & $(0.58)$ & $(1.73)$ & $(1.0)$ & $(0)$ \\
\hline
\end{tabular}

Implicit word association

Table 5.2

Mean encoding condition completions: implicit word association Standard deviations are in parenthesis.

\begin{tabular}{|l|c|c|c|}
\hline $\mathrm{n}=14$ per cell & Baseline & Read & Generate \\
\hline Experimental & $\begin{array}{c}2.46 \\
(1.08)\end{array}$ & $\begin{array}{c}2.86 \\
(2.14)\end{array}$ & $\begin{array}{c}3.21 \\
(2.42)\end{array}$ \\
\hline \multirow{2}{*}{ Control } & $\begin{array}{c}2.57 \\
(0.98)\end{array}$ & $\begin{array}{c}2.93 \\
(1.21)\end{array}$ & $\begin{array}{c}4.21 \\
(1.85)\end{array}$ \\
\hline
\end{tabular}

A 2 (Group: dysphoric and control) X 2 (Study Condition: studied and non studied) ANOVA revealed no significant main effect of Group ( $p>0.05$ ) however there was a significant main effect of Study Condition, $F(1,26)=4.89$ 
$\underline{p}<0.05$. This was shown by the higher study $(\bar{X}=3.30, \underline{S D}=1.90)$ mean when compared to the non studied baseline $(\bar{X}=2.51, \underline{S D}=1.03)$. There was no significant Group X Study Condition interaction, $\underline{F}(1,26)=.364, \underline{p}>0.05$.

A 2 (Group) X 3 (Encoding Condition: read, generate and baseline) ANOVA revealed no significant main effect of Group, $(p>0.05)$ however there was a significant main effect of Encoding Condition, $\underline{E}(2,52)=5.43, \underline{p}<0.01$, (refer Figure 5.1). Post hoc (scheffé) tests, collapsed across Group showed a significant difference between the generate $(\bar{X}=3.71, \underline{S D}=2.13)$ and baseline $(\bar{X}=2.51, \underline{S D}=1.03)$ conditions, $\underline{F}(2,52)=5.53, \underline{p}<0.01$ however there was no significant differences between the generate and read $(\bar{X}=2.89, \underline{S D}=1.67)$ conditions, $\underline{p}>0.05$ or between the read and baseline conditions, $\underline{p}>0.05$. There was no significant Group X Encoding Condition interaction, $\underline{F}(2,52)=$ $1.004, \mathrm{p}>0.05$.

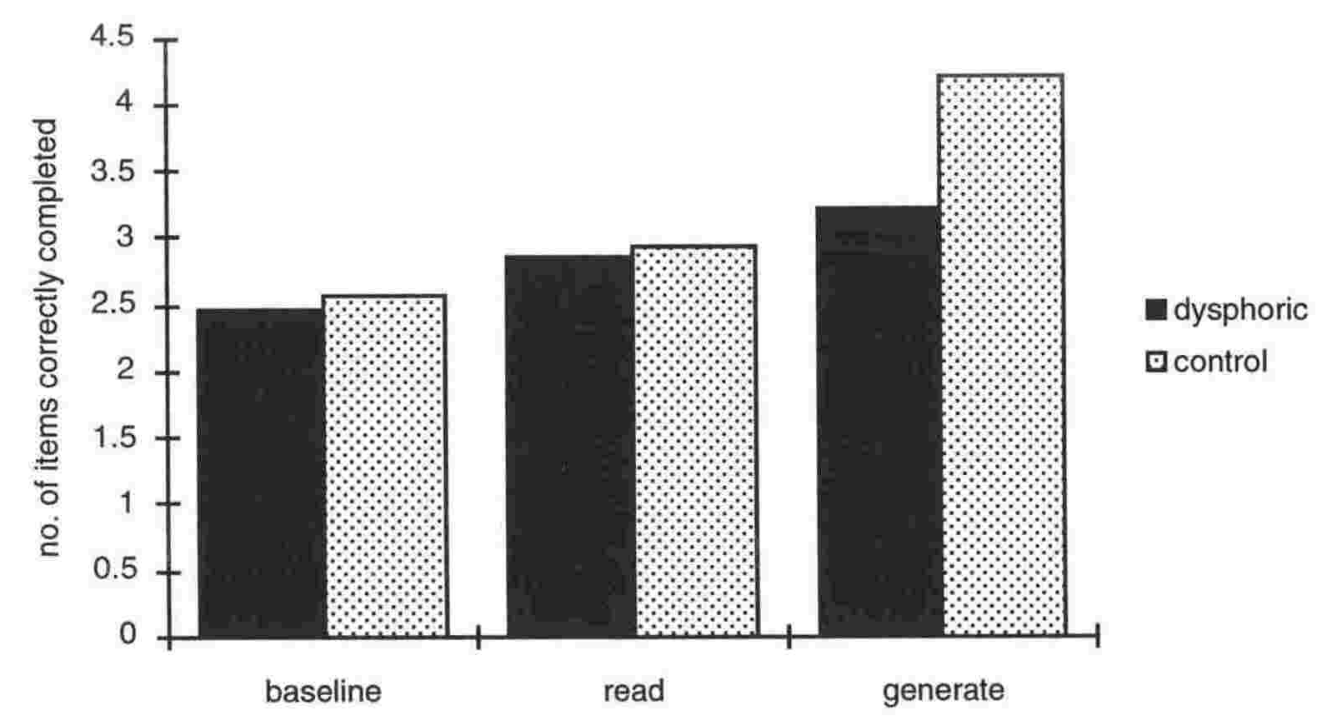

Figure 5.1 Mean correct completions - implicit word association

A 2 (Group) X 2 (Encoding Condition: read and generate) ANOVA was performed on studied data corrected for baseline guessing. There was no significant main effect of Group, $\underline{F}>0.05$, however there was a significant main effect of Encoding Condition, $\underline{F}(1,26)=6.29, \underline{p}<0.05$. This was shown by the 
better overall performance in the corrected generate condition $(\bar{X}=1.19, \underline{S D}=$ 1.10) when compared to the corrected read condition $(\bar{X}=.375, \underline{S D}=0.64)$. There was no significant Group X Study Condition interaction, $\underline{p}>0.05$.

\section{Explicit word association}

Table 5.3

Mean encoding condition completions: explicit word association Standard deviations are in parenthesis.

\begin{tabular}{|l|c|c|c|}
\hline$n=14$ per cell & Baseline & Read & Generate \\
\hline Experimental & $\begin{array}{c}1.57 \\
(1.24)\end{array}$ & $\begin{array}{c}4.71 \\
(2.46)\end{array}$ & $\begin{array}{c}7.57 \\
(2.24)\end{array}$ \\
\hline \multirow{2}{*}{ Control } & $\begin{array}{c}1.78 \\
(0.85)\end{array}$ & $\begin{array}{c}4.36 \\
(1.64)\end{array}$ & $\begin{array}{c}10 \\
(1.52)\end{array}$ \\
\hline
\end{tabular}

A 2 (Group: dysphoric and control) X 2 (Study Condition: studied and non-studied) ANOVA revealed no significant main effect of Group ( $\underline{p}>0.05$ ) however there was a significant main effect of Study Condition, $\underline{F}(1,26)=$ $173.72 \underline{p}<0.001$. This is shown in the higher study $(\bar{X}=6.66, \underline{S D}=1.96)$ mean when compared to the non studied baseline $(\bar{X}=1.68, \underline{S D}=1.04)$. There was no significant Group X Study Condition interaction, $\underline{F}(1,26)=1.18, \underline{p}>0.05$.

A 2 (Group) X 3 (Encoding Condition: read, generate and baseline) ANOVA revealed no significant main effect of Group, $\underline{F}(1,26)=4.144, \underline{p}>0.05$ however there was a significant main effect of Encoding Condition, $\underline{F}(2,52)=$ 114.08, $\underline{\mathrm{p}}<0.001$, (refer Figure 5.2 below). Post hoc (scheffé) tests, collapsed across groups showed a significant difference between the generate and baseline conditions, $\underline{E}(2,52)=117.56, \underline{p}<0.01$. There was also a significant difference between the generate $(\bar{X}=8.78, \underline{S D}=1.88)$ and read $(\bar{X}=4.53, \underline{S D}=$ 2.05 ) conditions, $\underline{E}(2,52)=42, \underline{p}<0.01$ and between the read and baseline $(\bar{X}$ $=1.67, \underline{\mathrm{SD}}=1.04)$ conditions, $\underline{\mathrm{F}}(2,52)=19.02, \underline{\mathrm{p}}<0.01$. 


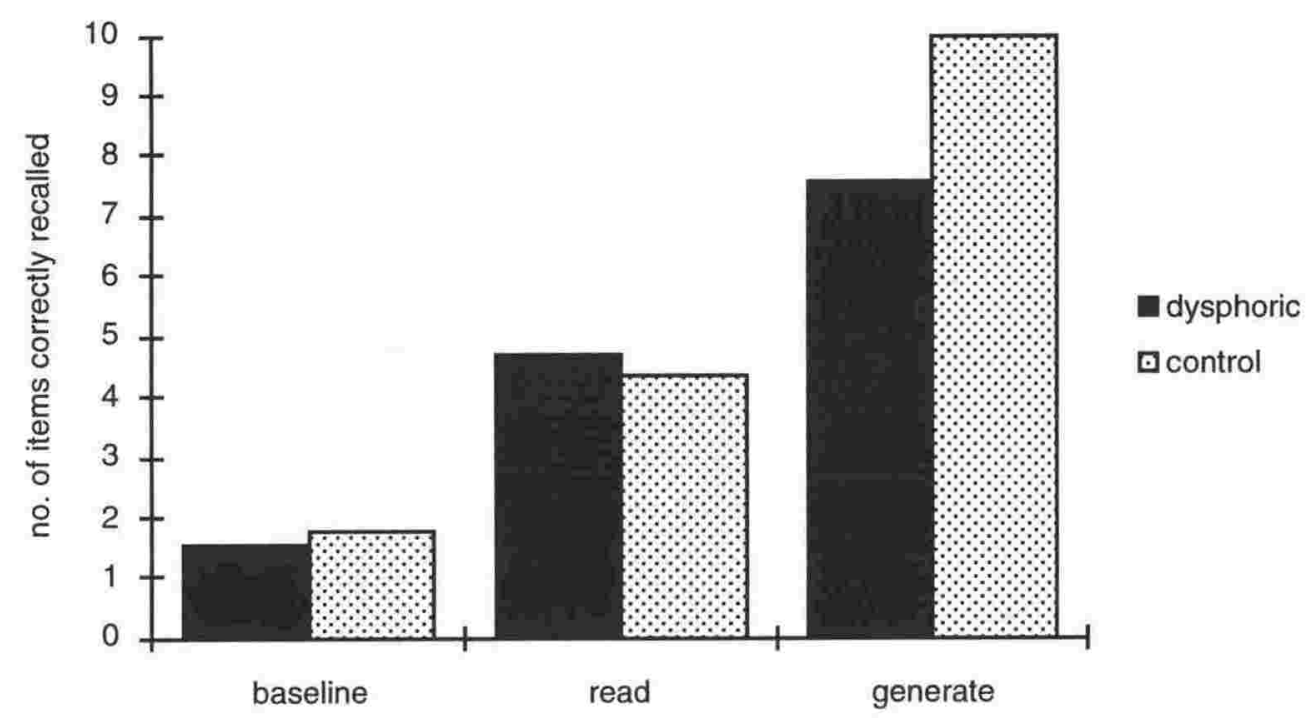

Figure 5.2 Mean correct completions - explicit word association

There was also a significant Group X Encoding Condition interaction, F $(2,52)=4.87, \underline{p}<0.05$. Post hoc (scheffé) tests, between group showed a significant difference between the dysphoric and control groups for the generate condition, $\underline{F}(2,52)=13.56, \underline{p}<0.01$, however there were no significant differences for the read $(\underline{p}>.05)$ or for the baseline condition $(p>$ 0.05).

A 2 (Group) X 2 (Encoding Condition: read and generate) ANOVA was performed on studied data corrected for baseline guessing. There was no significant main effect of Group ( $p>0.05$ ) however there was a significant main effect of corrected Encoding Condition, $\underline{E}(1,26)=71.11, \underline{p}<0.001$. This was shown by the better overall performance in the corrected generate condition $(\bar{X}$ $=7.11, \underline{S D}=0.83)$ when compared to the corrected read condition $(\bar{X}=2.86$, $\underline{S D}=1.0)$. There was also a significant Group $X$ Encoding Condition interaction, $\underline{E}(1,26)=7.64, \underline{p}<0.01$. A post hoc (scheffé) test performed on the corrected group results showed that there was a significant difference between the dysphoric and control group for the generate condition, $E(1,26)=9.61, p<$ 0.001 , however there were no significant differences for the read condition ( $p>$ $0.05)$. 
Furthermore a significant correlation was found between the BDI scores and performance in the explicit generate condition, $r=-.47, \underline{p}<0.01$. A significant correlation was also found between the DACL scores and performance in the explicit generate condition, $r=-.439, \underline{p}<0.05$. In both cases as scores on the depression inventory increased, performance in the explicit generate condition deteriorated.

\section{A Comparison between the implicit and explicit tests}

A 2 (Group: dysphoric and control) $\times 2$ (Test: implicit and explicit) $\times 3$ (Encoding Condition: read, generate and baseline) ANOVA revealed no significant main effect of Group, $\underline{E}(1,26)=3.47, \underline{p}=0.07$ however there was a significant main effect for Test, $\underline{E}(1,26)=42.64, \underline{p}<0.001$ which was reflected in the higher overall performance for the explicit test $(\bar{X}=5.0, \underline{S D}=1.65)$ compared to the implicit test $(\bar{X}=3.04, \underline{S D}=1.61)$. There was also a significant main effect for Encoding Condition, $\underline{F}(2,52)=92.92 \underline{p}<0.001$. Post hoc (scheffé) tests collapsed across Group and Test showed significant differences between the generate and baseline conditions, $\underline{F}(2,52)=123.3, \underline{p}<0.01$, between the read and baseline conditions, $\underline{F}(2,52)=18.92, \underline{p}<0.01$ and between the generate and read conditions, $\underline{F}(2,52)=45.62, \underline{p}<0.01$.

There was no significant interaction between Group X Test, $(\underline{p}>0.05)$. There was a significant interaction between Group X Encoding Condition, $\mathrm{F}$ $(2,52)=5.26, \underline{p}<0.01$. This is outlined in Table 5.2 below. A post hoc (scheffé) test collapsed across Encoding Condition showed that there was a significant difference between the dysphoric and control group for the generate condition, $E(2,52)=21.09 p<0.01$, however there were no significant differences for the read $(p>.05)$ or the baseline condition $(p>0.05)$. 
Table 5.4

Combined test encoding means by encoding type for experimental and control participants. Standard deviations are in parenthesis.

\begin{tabular}{|l|l|l|l|}
\hline Encoding condition & Baseline & Read & Generate \\
\hline Experimental & 2.01 & 3.8 & 5.4 \\
& $(1.16)$ & $(2.3)$ & $(2.33)$ \\
Control & 2.18 & 3.64 & 7.10 \\
& $(0.91)$ & $(1.42)$ & $(1.68)$ \\
\hline
\end{tabular}

There was also a significant interaction between Test $\mathrm{X}$ Encoding Condition, $\underline{F}(2,52)=51.35, \underline{p}<0.001$. This is outlined in Table 5.3 below. Post hoc (scheffé) tests collapsed across Group showed significant differences between the implicit and explicit tests for the generate condition, $\underline{F}(2,52)=$ 192.84, $\underline{p}<0.01$, for the read condition, $\underline{E}(2,52)=18.92, \underline{p}<0.01$ and for the baseline condition, $\underline{F}(2,52)=5.02, \underline{p}<0.05$. The interaction between Group $X$ Test $X$ Encoding Condition was not significant, $\underline{E}(2,52)=1.33, \underline{p}=0.27$.

\section{Table 5.5}

Implicit and explicit test encoding condition means

Standard deviations are in parenthesis.

\begin{tabular}{|l|l|l|l|}
\hline Encoding condition & Baseline & Read & Generate \\
\hline Implicit test & 2.51 & 2.92 & 3.71 \\
& $(1.03)$ & $(1.67)$ & $(2.13)$ \\
Explicit test & 1.68 & 4.53 & 8.85 \\
& $(1.04)$ & $(2.05)$ & $(1.88)$ \\
\hline
\end{tabular}

\subsection{Discussion}

Experiment 4 found that dysphoric participants recalled significantly less words from the generate condition in the explicit word associate cued recall test. No difference was found between groups in the implicit word association test. No differences were found in read priming for either test. The word 
associate cued recall test collapsed across groups was primed significantly more by the generate condition while no priming difference was found in the implicit word association test.

The most important aspect of Experiment 4 was the finding of an explicit conceptual deficit in a group of participants sourced from a student population. This finding adds to the findings of previous experiments which have also found significant results with dysphoric participants obtained from the same source. The mean BDI level found in Experiment 4 (20.64) is compatible to that of participants reporting moderate to severe forms of depression (see Vrendenburg et al.,1993). Since professional means were not used to diagnose depression, the exact status of these participants will not be known.

Whatever these students were experiencing it did result in a conceptual deficit, although this was only noted in the explicit version of the test. In comparing Experiments 1,2 and 4 this suggests that with the use of conservative selection criteria such as high cut off scores, high re-screen scores, and the use of secondary measures, differences in performance can be found using student participants.

A direct comparison between tests revealed significant differences in implicit and explicit test performance. That is collapsed across groups a significant difference was found between the generate condition used in the explicit test and the generate condition used in the implicit test. Likewise a significant difference was found between the implicit and explicit tests in the read condition. Finally a significant difference was found between the implicit and explicit tests in the baseline condition. This meets the requirements of the retrieval intentionality criterion. In other words it is unlikely that explicit contamination occurred in the implicit test.

The use of the same cues in both test versions, foil words, multiple interpolated tasks and large baseline blocks, were arguably an effective means of reducing or eliminating explicit contamination in implicit test performance. This is not to suggest that there was widespread contamination in Experiments 
$1-3$, however only Experiment 4 offered a direct check on the presence of any contamination.

Experiment 4 established that a conceptual deficit could be shown within an experiment that was designed to induce relatively 'pure' measures of implicit and explicit test performance. This finding provides extra validation for Experiment 3 which also found an explicit conceptual deficit. While Experiment 3 found an implicit conceptual deficit, Experiment 4 did not. However, in Experiment 3 the explicit test (free recall) was presumably more difficult than the implicit category association test. As a result task difficulty cannot be used to discredit Experiment 3, since a significant deficit was also found in a test that was most likely easier than the explicit test.

The inconsistent result between Experiments 3 and 4 could also add to the suggestion that there were important differences between participants diagnosed with a depressive disorder and participants obtained on the basis of self report inventories (Coyne, 1994). That is in dysphoria the mood state is not sufficient to produce an implicit conceptual deficit. However it is possible that the participants used in Experiment 4 may reveal such a deficit should their mood state further decline. Only further experimentation using both student and clinical participants at different levels and stages of depression can help answer this question. Even if an implicit conceptual deficit was found in an experiment using student participants this would still not rule out the possibility that there are still qualitative and empirical differences between student 'self reported' and clinical, depression.

Experiment 4 also provides both support and some difficulty for the original memory systems and processing models. The dissociation between intact implicit and impaired explicit test performance is not only supportive but could be easily explained by the memory system model. At the same time the use of the read - generate encoding manipulation to verify the conceptual status of these tests and to provide a measure of conceptual transfer was useful in predicting and explaining test performance. This manipulation showed that the depressive deficit was limited to transfer from the 'generate' condition while no 
difference in transfer from the read condition was noted. Such a finding suggests that the consideration of the type of processes used in test performance is an useful undertaking even if one primarily supports a systems model approach.

In other words in order to fully explain Experiment 4, a consideration of both memory systems and processes was required. For the processing model to fully explain Experiment 4, it must explain why the conceptual process supporting implicit test performance was not impaired, while the process behind explicit test performance was. Perhaps there are separate conceptual mechanisms supporting implicit and explicit test performance. The memory system versus processing debate will be expanded in the next section. 


\subsection{General discussion}

Experiments $1-4$ examined dissociations in several memory tests among dysphoric/ depressed students as well as participants diagnosed with a clinical depression. An important aspect of this research was the examination of the processing and memory systems models. For the processing model to be supported impairments should have been found in a test requiring predominantly conceptual processing while the perceptual tests should remain intact. For the memory systems model to be supported depressive impairments should have been limited to a test using explicit test instructions.

Intact perceptual processing was found across Experiments 1 - 4, in several tests using implicit test instructions and one task using explicit test instructions (refer table 6.1). Turning to the conceptual tests, Experiments 1 and 2 used dysphoric students and found intact conceptual processing in several explicit tests and one implicit test. Experiment 4 also used dysphoric students and found impaired conceptual processing in an explicit test and normal levels of performance in the implicit conceptual test. Experiment 3 used participants experiencing a clinical depression and found impaired conceptual processing for both the implicit and explicit tests. In general these results provide support for aspects of both the processing and memory systems models.

The use of the read - generate encoding manipulation showed that the tests used across Experiments 1 - 4 were mainly perceptually or conceptually driven as appropriate. That is most of the conceptual tests were primed significantly more by the generate encoding condition. No difference in priming from the read and generate conditions was found in the anagram solution test (this outcome was expected on the basis of previous experiments). The free recall test used in Experiment 3 showed no difference in priming between the read and generate conditions, however a floor effect appears to have occurred in this test. Most of the perceptual tests showed significantly more priming from the read encoding condition (some showed this only in the data corrected for 
Table 6.1: overview of Experiments 1-4

Exp. no Test

BDI mean dysphoric/depressed

result

Conceptual tests

One

implicit - category association explicit - semantic cued recall

14.56 dysphoric (student)

$\mathrm{F}<1$

$\mathrm{F}<1$

Two

explicit - free recall

16.50 dysphoric (student)

$p>.05$

\section{Four}

implicit - word association

20.64 dysphoric/depressed (student)

explicit - word association cued recall

$p>.05$

$p<.05^{*}$

\section{Three}

implicit - category association

explicit - free recall

25.4 depressed (clinical participants)

$$
\mathrm{p}<.05^{*}
$$

$\mathrm{p}<.05^{\star}$

14.56 dysphoric (student)

implicit - word fragment completion explicit - graphemic cued recall

\section{Two}

implicit - perceptual identification implicit - anagrams

16.50 dysphoric (student)

$\mathrm{F}<1 \bullet$

$\mathrm{F}<1 \bullet$

20.64 dysphoric/depressed (student)

no perceptual experiments

\section{Three}

implicit - word fragment completion

25.4 depressed (clinical participants)

$\mathrm{F}<1^{*}$

$\mathrm{F}<1 \bullet$

\section{Four}

Notes

1. in all cases the reported statistic is the group $X$ encoding interaction

2. * denotes a significant result

3. no differences were predicted for the perceptual tests. 
baseline guessing). Finally the graphemic cued recall test (Experiment 1) showed no difference in priming between the read and generate conditions.

\subsection{The effects of depression on implicit - explicit tests of memory}

Experiments 1 - 4 have provided further insight into the effects of depression and dysphoria on implicit and explicit tests of memory. Experiments 1 and 2 suggest that in mild mood states such as dysphoria there is no effect of depression on either implicit or explicit tests of memory. Experiment 3 suggests that in clinical depression an impairment can be found in both implicit and explicit memory tests. However Experiment 4 suggests that in less severe mood states explicit tests are impaired while implicit tests remain unimpaired. Taken as a whole these results suggest that depressive impairments are more likely to effect explicit test measures while implicit test measures are more robust to the effects of depression.

The above results are similar to those found in previous implicit memory depression research. Hertel and Hardin (1990) used university students with 'naturally occurring depression' and found no significant effect of depression on their implicit or explicit test measures. This finding is the same outcome as Experiment 1 and 2.

Danion et al. (1991) used depressed participants drawn from a clinical population and found normal implicit and impaired explicit test performance. This is the same outcome as Experiment 4 although in this later case participants were drawn from a student population. Experiment 3 used clinical participants and found an impairment in the explicit test although an impairment was also found in the conceptually driven implicit test. However Elliot and Greene (1992) tested participants drawn from a clinical population and found impairments in both their implicit and explicit tests.

Elliot and Greene (1992) acknowledge that impairments in implicit testing is unusual when compared to a number of similar experiments, for example Danion et al. (1991). They suggested this could be explained by differences in 
test procedure or in the mood state of participants used across these experiments.

Taken together the results of these experiments along with Experiments 1 - 4 suggest that explicit deficits are a common outcome in depressed mood states while the appearance of an implicit test deficit is less likely. Furthermore the appearance of both explicit and more particularly implicit test deficits are more likely to occur as the mood state of the participant deepens from dysphoria to a clinical depression.

These results also support a distinction between the processes tapped by explicit tests and those tapped by implicit tests. Roediger and MCDermott (1992) note that to date most research in this area supports this distinction, largely due to the finding that most explicit tests of memory are impaired in depression while most implicit tests are spared (see Danion et al.,1991 for a similar conclusion).

While the results of Experiments $1-4$ largely match those of previous experiments, Experiments 1 - 4 were also carried out to compare and contrast a number of different models. When these models are taken into account the interpretation of the above experiments is altered. It is to these models that the next section will turn.

\subsection{The processing model}

The present study provides some support and some difficulty for the processing model. The processing model has predicted that no impairment in perceptual processing will be found in memory impaired participants. Experiments 1,2 and 3 did not find any impairment in the implicit perceptual tests that were used. Also Experiment 1 did not find an impairment in the explicit perceptual test that was used. While this later result is supportive of the model, the most reasonable explanation of this result is the mild mood state experienced by the participants in Experiment 1. 
Turning to the conceptual tests, the processing model has predicted that conceptual impairments will be found in memory impaired participants. Explicit conceptual deficits were found in Experiments 3 and 4. Furthermore in Experiment 3 conceptual deficits were found in both the implicit and explicit versions of these tests. The findings of Experiment 3 are especially supportive of the processing model in that this dissociation was between the conceptual and perceptual processes and cut across the implicit - explicit distinction used by memory system models.

While Experiments 1 and 2 did not find any impairments in the conceptual implicit or explicit tests, a reasonable explanation would be the mild mood state experienced by the participants in these experiments. As a result these null results are not problematic for this model.

The use of the read - generate encoding manipulation across Experiments $1-4$ has provided an approximate measure of the contribution of perceptual and conceptual processes within each test. This manipulation has shown that the impairments found in Experiments 3 and 4 were restricted to transfer from the generate condition. In these cases impairments were restricted to generate transfer most probably due to the higher conceptual component in this condition (Blaxton, 1992 has suggested that impairments were more likely to effect the conceptually dominant generate condition). Furthermore the small level of transfer in the read condition found in most conceptual tests makes it difficult for between group differences to be revealed in that condition.

The findings of Experiments $1-4$ also demonstrate the utility of including a manipulation of perceptual and conceptual processes in a test design. The above results suggest that the manipulation of process type can be used to predict and explain the outcome of experiments using depressed participants with some accuracy. This is an improvement over past test designs which have not included this manipulation and as a result lack this predictive power.

There are a number of results across Experiments 1 - 4 which are problematic for the processing model. The processing model has difficulty explaining why the conceptual deficit found in Experiment 4 was only found in 
the explicit test. Traditionally the TAP framework does not attribute different properties to conceptual processing whether utilised under implicit or explicit test instructions (Blaxton, 1989,1992). As a result the framework fails to explain why implicit conceptual processes appear more robust (refer Experiments 1 and 4) than explicit conceptual processes.

It is also useful to compare Experiments $1-4$ to previous research in the light of the processing model. Danion et al. (1991) used an implicit test which is typically regarded as perceptual and an explicit test which is regarded as conceptual in processing type. Their impairment was restricted to the conceptual test which is a similar outcome to Experiment 3 . While this could be regarded as supportive of the processing model no perceptual explicit or conceptual implicit test was used as a point of comparison between these two models.

However there were a number of results less able to be accommodated by the processing model. For instance a number of experiments used a conceptual implicit test and found no depressive impairment (Hertel \& Hardin, 1990; Watkins et al., 1996). This finding is similar to Experiments 1 and 3 which suggests that conceptual priming can be reasonably robust and is only impaired in severe states of depression. These combined results add to the concern that the processing model must explain why explicit conceptual tests are more likely to be impaired than implicit conceptual tests.

Elliot and Greene (1992) found an impairment when they used an implicit test that was primarily perceptual in nature. Such an impairment is in contrast to the robust nature of the perceptual priming found across Experiments 1 - 4 and in a range of previous experiments, for example Blaxton (1992). This suggests that this result is highly unusual and may therefore be best explained by a variety of other factors, e.g. contamination by intentional processes. 


\subsection{The memory systems model}

The present study provides some support and some difficulty for the original memory systems model. As predicted by this model impairments were found in the explicit tests used in Experiments 3 and 4. To put this another way, in these experiments impairments were found when intentional retrieval instructions were used. Furthermore in experiments 1,2 and 4 no impairments were found in the implicit tests that were used. The results of Experiment 4 was especially supportive of this model since this follows the classic pattern of performance associated with this model, that is impaired explicit and normal implicit test performance. In the case of Experiment 4 this dissociation was found even though the test cues were held constant and as a result the same amount of information was available to support retrieval in each test. This finding suggests that depression only effects explicit or intentional test performance and is much less likely to impair implicit test performance.

While no impairment was found in the explicit tests used in Experiments 1 and 2 this can be explained with reference to the mild mood state experienced by the participants in these studies. As a result as in the case of the processing model, these null results are not problematic for this model in themselves.

There are a number of results across Experiments $1-4$ which are problematic for the original memory systems model. For instance this model has difficulty accounting for the implicit (conceptual) deficit found in Experiment 3, a process which is traditionally regarded as intact. Instead this finding is better accommodated by the processing model.

Experiments 1 - 4 can also be compared to previous research in the light of the memory systems model. The outcome of Danion et al. (1991) can also be used to support the original memory systems model. Rather than explaining this result with reference to the type of processes involved one could also explain this with reference to the operation of impaired episodic and intact semantic memory systems. Likewise Hertel and Hardin (1990) and Watkins et 
al. (1996) found no impairments in their implicit tests, even though several of these could be regarded as 'conceptual'. However Elliot and Greene (1992) found impairments in both of their implicit tests, an outcome problematic for the original memory systems model. This finding along with Experiment 3 suggests that participants with severe depression can show impairments in implicit test performance.

\subsection{Time for a combined model?}

This study provides some evidence to support the moving together of the processing and memory systems models. As noted above the results of these experiments have not supported one model outright over another. Rather a number of results have at times supported a particular model and at other times raised doubts about a models usefulness.

Traditionally the processing model states that only one form of conceptual or perceptual processing exists. In order for the processing model to account for the results of Experiment 4 it needs to assume that at least two forms of conceptual processing exist, one supporting explicit test retrieval and another implicit test priming. The results of Experiments 1,2 and 3 give some support to the suggestion that perceptual processing exists on a singular continuum. However if future experiments find that explicit perceptual tests are impaired in depressed participants up to four separate processes would be needed to account for these combined results.

Such an outcome would require changes to the current processing model to allow for the operation of multiple processes. There are a number of reasons to favour a combined memory systems and processing model. For instance in Experiments 3 and 4 the nature of the test instructions were an important determinant of participant performance, that is it was the use of intentional retrieval instructions which led to the test impairments in these experiments. Likewise previous depression - implicit memory experiments have noted this common dissociation. The memory systems model associates the 
use of different instructions with the operation of distinct memory systems. For instance the use of intentional retrieval instructions is associated with the operation of the episodic memory system.

Increasingly the processing model is being challenged by data supporting the division of human memory. For instance an increasing number of experiments are reporting evidence that perceptual and conceptual priming are dependant on different memory 'systems', for example Keane, Gabrieli, Fennema, Growdon and Corkin (1991); Tulving, Hayman and McDonald (1996). An increasing number of experiments also support the utility of a distinction between semantic and episodic memory (see Richardson-Klavehn \& Bjork, 1988; Schacter, 1987). There is also growing evidence that semantic learning can be dissociated from both episodic memory and perceptual priming, although this is largely based on dissociations observed in amnesic participants, (see Tulving, Hayman \& McDonald,1996).

These findings indicate that the processing model should start to take into account the influence of distinct memory systems on the operation of memory processes. Experiment 4 suggests that this distinction should be between a memory system which supports explicit processes and one that supports implicit processes. At another level this model should make more room for the role of intentional versus unintentional test instructions in predicting test performance.

At the same time the memory systems model is challenged by aspects of processing research. Until recently the memory systems model has been neutral in regards to the operation of distinct processes within a memory system. Experiments $1-4$ has challenged this by providing reasonable evidence that specific explicit or implicit tests draw on particular processes. For instance across Experiments 1 - 4 there was reasonable evidence to support a distinction between perceptual and conceptual processes in implicit memory testing. Experiments 1 and 2 showed that some implicit tests rely predominantly on perceptual processing. Experiments 1, 3 and 4 showed that other implicit tests rely primarily on conceptual processing. 
Furthermore perceptual processes were found to be uniformly robust to the effects of depression while conceptual priming can be impaired by severe depression (Experiment 3), whereas in dysphoric states it is intact (Experiments 1,2 and 4). This supports a distinction between conceptual and perceptual processes within the implicit memory system. The robust nature of the implicit perceptual tests and the finding that the implicit conceptual test can be impaired (Experiment 3) can suggest that each process is served by its own sub-system.

Experiments $1-4$ have also provided some evidence supporting a distinction between conceptual and perceptual processes in explicit test performance. For instance depressive deficits were found in several explicit tests that drew predominantly on conceptual processes. It is possible that no deficit will be found in explicit tests which rely predominantly on perceptual processes (see Blaxton, 1992:Expt.1). If this is the case, this suggests that the explicit or episodic memory system can support the function of a number of different processes each of which varies in its vulnerability to depression. Partly due to the lack of significant results for the explicit - perceptual test (Experiment 1), further research will be required to ascertain whether these processes are each served by separate sub-systems or within the same episodic system.

The above results suggest that there would be an increase in explanatory power if aspects of the processing model were incorporated into the memory systems model. That is the memory systems model would better account for the results of Experiments 1 - 4 if it took into account the operation of distinct processes within a memory system framework.

\subsection{The utility of the revised memory systems model}

In recent years the memory systems model has undergone a number of revisions. This has resulted in the postulation of a number of distinct systems each of which specialises in a specific form of processing. For instance Tulving and Schacter (1990) propose that implicit perceptual priming is served by the Perceptual Representation System (PRS) and implicit conceptual priming by 
the modification of semantic memory. Explicit conceptual priming is supported by the episodic memory system while the possible input of the PRS into episodic memory could be the source of perceptual explicit test recall.

The revised memory system model (Schacter \& Tulving,1994) is able to accommodate aspects of the present study that create difficulties when these are interpreted in terms of individual models. For instance the revised model makes a distinction between conceptual (semantic) and perceptual (PRS) implicit priming. As a result the revised model is able to accommodate the results of Experiment 3 which found a dissociation between the two implicit tests that were used.

The model also attributes the use of separate conceptual processes within the semantic and episodic systems. Such a view can accommodate the results of Experiment 4 which found a dissociation between the conceptually driven implicit and explicit tests. The model also infers that explicit retrieval can draw on a predominantly perceptual code and as a result support retrieval for explicit perceptual tests.

The revised model is also able to explain previous depression - implicit memory research (Danion et al., 1991). This model is able to accommodate those experiments which found intact conceptual implicit test performance (Hertel \& Hardin, 1990; Watkins et al., 1996) even when the explicit test was impaired. However this model cannot explain why Elliot and Greene (1992) found an impairment in their implicit perceptual test. This is largely because this model assumes that the PRS is a robust system not subject to impairment.

While this model represents an useful advance on the original memory systems model it would be a mistake to return to explaining differences in implicit - explicit test performance solely in terms of the operation of distinct memory systems, even if it is acknowledged that these each draw on a particular process. To put this another way the use of a manipulation such as the read -generate encoding manipulation should be routinely included in future test designs to verify the place and role of different processes in explaining test performance. 
There are a number of areas where the revised model lacks specificity. For instance the model is yet to show the precise relationship between the PRS and the semantic or episodic memory system. Experiment 1 and Blaxton (1992) did not find impaired recall in a perceptual explicit task. According to Schacter and Tulving the PRS system alone does not support explicit recollection. As a result this model needs to explain which mechanisms support explicit perceptual tests. For instance does the output of the PRS feed directly into the episodic system or must it be resolved first (or in parallel) by the semantic system where it is related to other pre-existing concepts or associative relations?

To date memory systems theorists have provided little detail on which memory systems should be effected by depressive deficits, apart from the commonly cited episodic system. If explicit perceptual tests are found to be intact in depressed participants, the revised model needs to predict and explain this. The model should also incorporate the finding that implicit conceptual tests can be impaired. As a result models of episodic and semantic memory need to be revised to take into account the possibility that they can be intact or impaired respectively.

\subsection{The integration-activation model}

As previously noted a number of alternative models have been put forward to explain the effects of depression on implicit - explicit memory tests. A number of authors have postulated the activation - elaboration model to account for dissociations in depression - implicit memory experiments, for example Danion et al. (1991). More recently this model has been refined so that the concept of integration has replaced that of activation (Graf \& Gallie, 1992; Graf \& Ryan, 1990).

The revised model proposes that integration primarily unifies the features of a target into a co-ordinated whole while elaborative processes link or associate a target with other mental representations. At study typically both 
integrative and elaborative processes are engaged. Yet at test implicit tests primarily engage integrative processing and explicit tests engage elaborative processing.

The model does not distinguish between perceptual or conceptually driven processes whether these are used during implicit or explicit testing. For instance all implicit tests whether based on word fragment cues or category association cues are regarded as depending primarily on integrative processes (Graf \& Gallie, 1992).

If all explicit tests draw primarily on elaborative processes they should be primed significantly more by the elaborative 'generate' condition, likewise if implicit tests are primarily integrative in nature they should be primed significantly more by the integrative 'read' condition. Yet across Experiments 1 4 this was not always the case (see also Blaxton, 1989, 1992) in that perceptual explicit tests were also significantly primed by the read condition (Experiment 1) and conceptual implicit tests by the generate condition (Experiment 1, 3 and 4).

As a result the model needs to explain why an implicit test which is defined as integrative can be primed significantly by an elaborative (generate) encoding task. Likewise the model needs to explain why a perceptual explicit test can be primed significantly more by an integrative read encoding manipulation. On the other hand the model was supported when an elaborative (explicit) test was primed significantly more by a generate (elaborative) study manipulation. Likewise when an integrative (implicit) test was primed significantly more by a read (integrative) study manipulation.

The model also proposes that deficits in conditions such as ageing (and by implication, depression) occur due to a decline in elaborative processes. Integrative processes are spared due to their 'automatic' nature. However in Experiment 3 a deficit was found in the implicit conceptual task despite the models assumption that these tests draw on integrative processing and therefore should be immune to deficits. This suggests that until this model can 
incorporate and explain the use of a process manipulation such as the read generate manipulation its usefulness is limited.

\subsection{Resource allocation model versus cognitive initiative model}

A number of authors have cited the resource allocation model as an explanation for the effects of depression on human cognition (Ellis \& Ashbrook, 1988). In brief this theory assumes that deficits occur in test performance since depression 'ties up' a portion of the limited pool of cognitive resources that are used in performing cognitive tasks. Furthermore the deeper the depression and I or the more complex the task the poorer the participants performance (Ellis and Ashbrook, 1988).

In contrast to this model Hertel and her colleagues, for example Hertel and Hardin (1990) put forward the cognitive initiative model. This model proposes that the full range of cognitive resources are available to the depressed. Instead impairments are caused by a reduction in cognitive initiative. Hertal and her colleagues note that when instructions are given to depressed participants to cue appropriate strategies, impairments are not 
perceptual tests. At first glance these tests appear similar and tend to be introduced by similar types of instructions. Yet according to this model less initiative is shown towards the conceptual test. This model also has the difficulty of trying to cue an appropriate strategy to the depressed participants without making the test easier (in comparison to the control participants) or giving away the nature of the implicit test.

The resource allocation model is supported by the finding that impairments appeared in the more demanding tasks, for example in the free recall and category cued recall tests (Experiments 3 and 4). Likewise as the mood level changed from dysphoria to possible depression to clinical depression the number of impairments also increased (cf. Experiments 2, 3 and 4).

One aspect of the resource allocation model that could be potentially difficult for interpreting the results of Experiments $1-4$ is test difficulty. In Experiment 3 the implicit conceptual test was impaired while the implicit perceptual test was not. One explanation for this could be that conceptual tests are in themselves more difficult than perceptual tests. That is certain tasks use up more capacity than other tasks. If this is the case then this is a confound with models which propose that deficits occur due to the reduced availability of a process or system to perform the test.

Yet if it is assumed that a certain test is more difficult (for instance an explicit test) than another test (for instance an implicit test) then presumably this difficulty will be experienced by both the control and depressed participants. Yet depressed participants routinely perform worse than controls especially on explicit tests. This suggests that there is a mechanism extra to test difficulty that is disrupting the depressed participants ability to perform these tests. While it could be suggested that depressed participants find certain types of test more difficult than controls this is more a description of a symptom than a cause. In this case some mechanism needs to be advanced to explain why depressed participants find certain types of test more difficult than controls. One such mechanism could be reduced cognitive control. 
Since depressed and control participants are exposed to the same test items one outcome of this is that it is acceptable to compare depressed and control participants performance even across tests of varying difficulty. Yet there is still a methodological problem if overall both groups find one type of test more difficult than another (Roediger \& McDermott, 1993). If this is the case then differences in implicit - explicit performance could be explained with reference to task difficulty rather than because they reflect the operation of distinct mental processes, for example automatic versus controlled processing. In order to gain more insight into this question more research on the exact 'cognitive load' of different tests needs to be undertaken. In the meantime test designs which seek to equate test conditions and difficulty must be preferred over those that do not. When this is done there is reasonable evidence to suggest that explicit tests on the whole measure different forms of mental processes than those tapped by implicit tests (Jacoby, 1991).

\subsection{Problems}

Across Experiments 1 - 4 a number of problems were experienced. Issues relating to the diagnosis of depression and the use of the BDI in this type of research will be discussed in a later section.

Firstly across Experiments 1 - 3 no check was included to monitor explicit intrusion into implicit test performance. Without such a check any deficit in implicit test performance becomes potentially difficult to explain with confidence. Ideally such a check should have been included in Experiments 1 3 through the use of the retrieval intentionality criterion. The criterion was not included in these experiments primarily due to a desire to base these on the tests and methodology used by Blaxton $(1989,1992)$ and other implicit memory - depression research, for example Hertel and Hardin (1990).

The inability to control for differing response strategies between and within tests is a particular problem in interpreting the finding of the implicit deficit found in Experiment 3 . Was this due to an impairment in implicit conceptual 
processing or contamination by explicit processes? Although no firm conclusion can be reached on this point it is likely that only a small level of explicit contamination was experienced across Experiments 1 - 4. Certainly no significant explicit contamination was noted in Experiment 4 which used the retrieval intentionality criterion as a check for this. Also it is very unlikely that participants will realise the nature of an implicit test straight away or that this state of awareness will occur in all participants (Graf \& Schacter, 1985). As a result explicit contamination should be held at a small or non-significant level. However in order to remove doubt on this issue a design modification such as the retrieval intentionality criterion should be included in future test designs.

A related issue was that across Experiments 1 - 3 a number of different tests were compared which confounded task difficulty as well as processing status. For example in Experiment 1 the semantic cued recall test was compared with the graphemic cued recall test as the two explicit tests. Had an impairment been found in the former it would have been difficult to conclusively state whether this was due to an impairment in a particular type of processing or because the first test is more difficult than the later test. Such a confound again demonstrates the utility of using a test design that holds cue information as similar as possible over both the implicit and explicit tests (see Experiment 4) since this reduces test difficulty as a confounding factor. Furthermore in order to manipulate process form, separate perceptual and conceptual test versions should be compared, most probably in separate experiments.

Experiments 1 - 4 used relatively few participants which at times resulted in low power. The use of a design where participants completed only 2 out of 4 possible tests contributed to this (Experiment 1). However the use of low participant numbers is not unusual in this area of research where typically depressed participants and even dysphoric students can be difficult to select and recruit. Elliot and Greene (1992), for instance used 10 participants and Hertel and Hardin (1990) 12 participants. Although none of these authors raised participant numbers as a concern ideally the findings of Experiments 1 4 should be replicated or re-examined before firm conclusions can be reached. 
In the meantime it seems reasonable to suggest that for participants experiencing BDI levels similar to those obtained in Experiments 1 and 2 that deficits may or may not occur even with larger numbers of participants. In contrast deficits are very likely to occur in experiments using clinical participants or those using high BDI cut off scores, even if fewer participants are used.

\subsection{Future research}

The findings of Experiments $1-4$ lead to a number of future research directions. An important issue is whether explicit perceptual tests are spared or impaired in depressed participants. In order to test this, a design similar to Experiment 4 should be used, except perceptual cues would be presented to the participants. A potential problem is that no difference will be found in either test (the outcome which Blaxton, 1989, 1992 would predict). Such an outcome could be confounded with an explanation suggesting that the participants mood state was insufficient to produce the appearance of a deficit (see Experiments 1 and 2). The use of participants drawn from a clinical population and a comparison with a series of conceptual tests may be means of overcoming this difficulty.

An important implication of Experiments 1 - 4 is the suggestion that the processing model should make more room for the role of memory systems in predicting test performance. However only Experiment 4 has provided direct evidence for this. This suggests that further experimentation is required to explore this issue. The repetition of Experiment 1 using a sample drawn from a clinical population will be an ideal starting point for this. The finding that, for instance explicit perceptual tests are impaired in depression will add weight to combining the memory systems and processing models.

As a general point future test designs should seek to test more specific predictions about the kinds of processes and systems involved in human cognitive performance. The exact relationship between these two concepts also requires ongoing exploration. Furthermore the specific relationship between the 
test designs and cues used in this area of research and the operation of specific systems and processes will require ongoing exploration. This should also include a systematic evaluation of test difficulty and the role this has in determining the presence of impairments across a range of tests. As these experiments are undertaken more specific loci for the effects of depression should be uncovered.

An alternative future research direction is Jacoby's (1991) process dissociation procedure (see also Jacoby, Toth \& Yonelinas, 1993). Using the principle of opposition this model provides a measure of intentional (those under conscious control) and automatic uses of memory in implicit and explicit measures of memory. It would be very useful to gain a measure of these processes within implicit - explicit tests of memory or within an encoding manipulation such as the read-generate manipulation. This is because without an independent measure of these processes it remains conjecture as to the extent to which these processes contribute to test performance. For instance the finding that implicit conceptual or perceptual tests largely draw on the operation of automatic processes will add weight to the finding that intentional or controlled processes do not contribute to the performance of these tests.

\subsection{Other clinical groups}

Experiments 1 - 4 were largely designed to test the effects of depression on the processing and memory systems models of memory and in so doing reveal which model provides the best means of detailing these effects. This has led to the postulation of a model where a number of seperate memory systems each specialise in processing a particular process, for example the implicit perceptual system (described in the wider literature as the PRS). A number of similar experiments to Experiments 1 - 4 have been carried out using a range of other clinical participant groups. It is an interesting question as to the extent to which the results of these experiments are congruent with those of Experiments $1-4$. 
Cermak, Verfaellie and Chase (1995) tested amnesic participants using conceptual implicit and explicit tests with matching cues (they also tested a groups of participants with matching perceptual tests). They found that the use of intentional retrieval instructions led to impaired performance in both the implicit and explicit tests rather than the nature of the processing requirements. Similarly Vaidya, Gabrieli, Keane and Monti (1995) used a study modality manipulation (visual - audio) to manipulate study process across a range of perceptual and conceptual explicit and implicit tests. Vaidya et al (1995) also used the same cues for their implicit and explicit tests. They found that the deficits occurred in the explicit tests while the implicit tests were unimpaired. In other words the type of process had no bearing on the appearance of deficits.

While the above results are problematic for the processing model and supportive of the memory systems model the results are not always nearly as clear cut as above. For example Keane, Gabrielli, Fennema, Growdon and Corkin (1991) found normal priming levels on a implicit test of perceptual identification and impaired performance on a explicit test of recognition. At times implicit conceptual tests are found to be impaired (Squire, Shimamura \& Graf, 1987) and less often implicit perceptual tests are also impaired (Cermak, Verfaellie \& Letourneau, 1993).

In a review of previous amnesia - memory research Vaidya et al (1995) note that processing differences invoked by different conceptual priming tasks may explain why conceptual priming appears intact in global amnesia and impaired in epileptic participants (which Blaxton, 1992 used for her experiments). Some forms of processing task are intact in amnesia, for instance word association and category exemplar generation (typically these are implicit tests). Others such as answering general knowledge questions (typically an explicit task) are impaired in amnesia.

Further differences in test performance can be found when one compares amnesics with participants with Alzheimer's disease. While global amnesics are typically unimpaired in performing many implicit conceptual tests Alzheimer's participants are routinely impaired in these tests (see Maki, 1995 
for a full review). Maki (1995) notes that Alzheimer's participants are typically unimpaired on perceptual implicit tests (her review does not discuss explicit tests).

While the common finding of robust explicit deficits and unimpaired implicit performance appears to contravene the findings of Experiment 3 there are clear differences between amnesic participants and participants experiencing a mood state such as depression. Amnesic participants have typically suffered irreversible damage to medial-temporal or diencephalic brain structures (Maki, 1995) where as depressed participants have typically not suffered this damage. Cermak, Verfaelie and Chase (1995) state that damage to these regions results in an impaired ability to use conscious or controlled processes leading to deficits on all explicit tests, whether the source of information is perceptual or conceptual in nature.

It is interesting that in severe depression (Experiment 3 ) the implicit conceptual test was impaired while in amnesia this type of test is often intact (although as previously noted impairments can occur). This can suggest that the results of Experiment 3 reflect something specific to depression that is not always apparent in amnesia, or that the Experiment 3 finding was invalid. The finding that conceptual implicit tests are commonly impaired in Alzheimer's (Maki, 1995) does however add weight to the findings of Experiment 3 and to the suggestion that different subsystems support conceptual and perceptual implicit priming.

In sum the findings of research with different clinical groups is a useful adjunct to the findings of Experiments $1-4$. While some of the experiments reveal different patterns of deficits to those found in Experiments 1 - 4 this is most likely explained by differences in brain damage/ impairment as well as differences in test design. It is interesting that some clinical groups are congruent to the findings of Experiments 1 - 4 which may suggest the involvement of common brain structures, although this will require further systematic examination. Perhaps most interesting of all, most of these 
experiments show the value of examining different types of processes within a memory system framework.

More recently researchers who specialise in the study of amnesia support the consideration of different types of processes within a memory systems account (see Gabrieli, 1995). Cermak, Verfaelie and Chase (1995), for instance freely suggest that amnesics commonly retain conceptual and perceptual fluency. Maki (1995) also suggests that the implicit dissociation between perceptual and conceptual processes adds weight to the suggestion that these draw on different systems. Gabrieli (1995) adds that one outcome of this new model is that in future equal weight should be assigned to defining processes as to delineating neural networks.

\subsection{The use of student participants as analogues for clinical depression.}

The findings of Experiments 1 - 4 also raise a number of issues concerning the means by which depressed or dysphoric participants were selected. As previously noted in Experiments 1 and 2 participants were selected via the use of the BDI yet no evidence of impairments were found in these participants. These findings raise the question of the adequacy of using students selected via self report measures as analogues for clinical depression.

The use of the BDI to select participants from student populations continues to be the subject of ongoing debate (Gotlib, 1984). Coyne (1994) notes that in the late seventies and early eighties critics started to challenge psychologists excessive reliance on college students selected on the basis of self report inventories to test hypotheses about clinical depression. Depue and Monroe (1978) state that the main area of concern is whether the 'state' represented by students scoring above a certain threshold on a measure such as the $\mathrm{BDI}$, represent a form of depression with clinical depression representing an extreme end or a different kind of mood state altogether, for instance general distress. 
If there is doubt as to what these students are experiencing then this calls into question the adequacy of developing models based on a sample which is qualitatively and quantitatively different from the clinically depressed (Coyne, 1994). If proponents of the former position are to be believed student participants do not differ substantially in kind from clinically depressed participants, but represent a different state altogether.

In comparing Experiments 1,2 and 4 with Experiment 3, an implicit conceptual deficit was only found in the group diagnosed with a clinical depression. Furthermore few deficits were noted in the student sourced experiments (Experiments 1,2 and 4) while the clinical participants exhibited marked impairment in the conceptual tests used. These varied findings suggest that whatever the university student participants were experiencing, differed not only in depth of depression but in kind as well.

However it is possible that an explicit or implicit conceptual deficit would have been found in a student group recording higher scores on the BDI. Alternatively an implicit conceptual deficit may not have been found in the clinically depressed during a later or recovery stage of their illness. As a result depth or stage of illness is confounded with 'kind' of depression. Despite this the results across Experiments $1-4$ were clear and the status of the depressed participants in Experiment 3 well known. It should be reasonable to conclude that student dysphoria does not represent a milder version of clinical depression but instead represents a different kind of condition.

As a result the findings of Experiments 1,2 and 4 should not be used to build models of depression for the clinically depressed, even though they can be a suitable model for student dysphoria. However it should be acknowledged that further in depth research will be needed on the exact similarities and differences between the state experienced by students selected using the BDI and those diagnosed as clinically depressed. Once more is known then it should be possible to extrapolate aspects of the results of studies using dysphoric students to models of clinical depression, with greater confidence. 
The use of the BDI as a means of classifying students into depressed and non-depressed groups has become a point of contention in recent years (Coyne, 1994). Clarification of this issue is important, for instance an easy acceptance of inferior participant classification methods could lead to hampered acceptance of psychological models of depression outside its field (Coyne, 1994).

A number of authors have noted that the BDI was never designed as a diagnostic instrument but rather was designed to estimate the severity of illness in participants already diagnosed with a primary depressive disorder (see Depue \& Monroe, 1978). Furthermore Coyne (1994) notes that the BDI was not designed specifically for use with students and that the conventional cut off commonly associated with this instrument have not been validated for use in this population (see Beck, Steer \& Garbin, 1988).

A further difficulty is that elevated scores on these scales could result from a number of factors other than depression, in other words there are no unique symptoms to depressive disorders. For example an elevated score could result from a grief reaction, loss of a significant other, low self esteem or another psychiatric condition. In the absence of other sources of background information, an elevated score on the BDI alone cannot be interpreted in terms of diagnostic concerns (Depue \& Monroe, 1978).

Furthermore participants do not have the relative objectivity or clinical insight of a trained psychologist in rating the severity of their symptoms (Depue \& Monroe, 1978). A cut off score of nine or 12 can be achieved in a wide variety of ways, including the strong endorsement of three or four items, which collectively may have little to do with the experience of depression, but may relate instead to another condition, medical complaint or a state of general sadness.

BDI scores obtained from student samples also typically show instability (Stone \& Neale, 1984) and tend to reflect the experience of symptoms over a 
short time period, for example one to two weeks. Participants with a diagnosis of a clinical depression often experience deliberating symptoms for a period considerably longer (Angst, 1986), suggesting that whatever student participants are experiencing can be fleeting and transient. Student participants also typically score lower than most clinical participants on self report measures, however research has shown that even high scoring student participants are not always depressed (see Deardoff \& Funabiki, 1985; Hammen, 1980; Oliver \& Simmons, 1984).

In contrast Vrendenburg et al. (1993) have defended the use of the BDI as a means of obtaining a depressed student sample, although they acknowledge the use of a single presentation is far from ideal. In doing so they criticise a number of studies used by Coyne (1994) to raise questions concerning the use of the BDI. For instance they criticise Gotlib (1984, cited in Vrendenburg et al.,1993) for not taking into account the high confound (i.e. overlapping item content) between the BDI and many other scales. It is therefore not surprising that the BDI will show high correlation's with other measures, including self distress. Furthermore they cite a number of studies which they claim suggest that both depressed college students and depressed psychiatric patients experience general distress as part of their illness (see Mook, Van der Ploeg \& Kleijn, 1990, cited in Vrendenburg et al.,1993).

Vrendernburg et al. (1993) also argue that with the use of appropriate measures (which can include the use of the $\mathrm{BDI}$ ) researchers are able to isolate relatively pure groups of depressed and anxious students and that it is also possible to be able to differentiate types of depression in a college student sample. For instance Depue, Krauss, Spoont and Arbisi, (1989) were able to identify students who met the criteria for unipolar depression and bipolar depression. This raises the issue that should be always possible to find students who are genuinely depressed. Coyne, (1994) does not suggest that students never become depressed, rather the issue is the adequacy of the means used to find and select these students. 
Vrendenburg et al. (1993) do acknowledge the use of the BDI alone has its limitations and that best practise would involve obtaining diagnostic information through other means as well, such as a semi-structured interview. When these means are not available they suggest a number of alternative measures to increase the reliability of the sample. Ideally future student research should incorporate these measures. This should include the use of multiple measures during the group classification phase instead of a reliance on a single measure. In administrating the BDI they suggest a number of improvements. For instance they recommend rewording a number of items to capture a broader range of somatic symptom expression (p. 331). They also recommend the use of $2 \mathrm{BDI}$ screens, the use of high cut-off scores (16 is suggested) and the requirement that participants score a minimum number of items over a minimum time period to be included in the 'depressed' group.

Although these recommendations represent an improvement over a single presentation of the BDI, Vrendernburg et al. (1993) are not able to guarantee that participants selected with the use of the BDI are diagnosable with a clinical depression, no matter how many self report measures are used. This can only be done by a clinical professional using appropriate measures (Coyne, 1994).

While doubt remains as to what high scoring student participants are experiencing Hertel and Knoedler (1996) suggest that it is appropriate to describe these participants as dysphoric rather than depressed. Here care must be taken to distinguish dysphoria as a label for 'what ever participants selected on the basis of the BDI are experiencing' and dysthymic disorder which is a diagnosable form of depression, characterised by a general depressed mood experienced most days over a 2 week period. Partly on this basis, Coyne (1994) prefers the term distressed or general distress, although provided no claim is made that there has been a diagnosis, the term dysphoria should be acceptable. 


\subsection{Closing summary}

Experiments 1 - 4 were largely carried out to examine the effects of depression on the memory systems and processing models of memory. In so doing it was hoped that this would show which model provided the best fit to the results of these experiments and show more accurately the loci of depressive impairments.

Experiment 1 tested dysphoric students on 2 explicit and 2 implicit tests of memory ( 1 test in each pair were perceptual and the other conceptual). During encoding the read - generate encoding manipulation was used to verify the processing status of each test accordingly. Experiment 1 did not find any dysphoric deficits on either the memory systems or processing dimensions that were tested.

Experiment 2 attempted to increase the likelihood of finding a significant result by reducing the cue information available at test. This was done by using tests which provided low or minimal levels of cue information, for example the perceptual identification test. No significant dysphoric impairments were found in this Experiment (university students were also used as participants).

Experiment 3 used 2 conceptual tests, one implicit and the other explicit. These were compared against a perceptual implicit test. Patients with a diagnosed major depressive disorder were used as participants. Experiment 3 found depressive impairments in both the implicit and explicit conceptual tests while no difference was found in the implicit perceptual test.

However Experiments 1 - 3 (as well as previous implicit - explicit memory depression research) have compared a number of tests which differ in test difficulty. In effect this confounds task difficulty with the particular dimension that is being examined. In contrast Experiment 4 presented the same cues across both tests to each of the participants and only varied the instructions that were used. Experiment 4 compared two conceptual tests, one using implicit retrieval instructions and the other explicit recall instructions. Dysphoric 
university students were used as subjects, a dysphoric impairment was found in the explicit test.

The above results provide some support and raise difficulties for both the processing and memory systems models. For instance the processing model was supported by the finding that in Experiment 3 both conceptual tests were impaired and the perceptual test was intact. However the processing model has difficulty accounting for the findings of Experiment 4 where only the explicit test was impaired. In contrast the memory systems model was supported by the finding of Experiment 4 and has difficulty explaining the intact implicit test in Experiment 3.

Greater sense of Experiments 1 - 4 can be made if aspects of the processing and memory systems models are combined. That is the results of these experiments can be more easily explained if it is assumed that different memory systems each specialise in the processing of particular kinds of information, for instance conceptual information. The revised memory systems model is one means of doing this and as a result is able to explain the findings of Experiments 1-4 with relative ease compared to when the systems and processing models are held seperate.

These findings also provide extra detail on the effects of depression/ dysphoria on human memory. For instance Experiments 1, 2 and 3 suggest that dysphoria has no effect on implicit tests but can effect conceptual explicit test measures. Clinical depression can lead to impairments in both conceptual implicit and explicit test measures. Perceptual implicit tests appear robust to the effects of both clinical depression and dysphoria while future research will need to determine whether explicit perceptual tests are effected by either condition.

Experiments 1 - 4 have also raised a number of more general issues of test design. Had singular versions of implicit and explicit tests been compared then it would not have been possible to examine the relative contribution of different processes or memory systems to memory performance. This suggests that for greater predictive power, future test designs will need to manipulate a range of measures at both encoding and test. Furthermore every effort should 
be made to equate the look and feel of these tests and only vary test instructions.

Future experiments will also need to be careful in the way they select and label student participants. While every effort should be made to use depressed participants where this is not possible it is acceptable to recruit dysphoric participants such as university students provided the limitations from this source are made clear. Furthermore when students are used the use of several measures, multiple presentations and question sheets designed to exclude a number of similar disorders should help recruit a reasonably homogeneous sample.

While Experiments 1 - 4 represent an advance on previous implicit explicit memory research much is still yet to be done. For instance it remains an open question as to whether explicit perceptual tests are subject to impairments in depression. However in the meantime it is hoped that the contribution of Experiments 1 - 4 will, if only in some small way lead to a greater understanding of the effects of depression on memory; and as a result contribute to greater understanding of and by those finding themselves facing the despair of depression. 


\subsection{References}

American Psychiatric Association. (1994). Diagnostic and statistical manual of mental disorders (4th ed.). Washington, DC: Author.

Angst, J. (1986). The course of affective disorders. Psychopathology, 19, 47 - 52.

Bauer, L. (1993). Written corpus of written New Zealand English. Wellington: Victoria University, Department of Linguistics.

Bazin, N., Perruchet, P., De Bonis, M., \& Feline, A. (1994). The dissociation of explicit and implicit memory in depressed patients. Psychological Medicine, 24, 239 - 245.

Beck, A. T. (1967). Depression: Clinical, experimental and theoretical aspects. New York: Harper \& Row.

Beck, A. T. (1970). Depression: Causes and treatment (1st ed.). Philadelphia: University of Pennsylvania Press, 1970.

Beck, A. T., Steer, R. A., \& Garbin, M. G. (1988). Psychometric properties of the beck depression inventory: Twenty five years of evaluation. Clinical Psychology Review, 8, 77 - 100.

Beck, A. T., Ward, C. H., Mendelson, M., Mock, J., \& Erbaugh, J. (1961). An inventory for measuring depression. Archives of General Psychiatry, 4, 561 - 571.

Blaxton, T. A. (1989). Investigating dissociations among memory measures: Support for a transfer appropriate processing framework. Journal of Experimental Psychology: Learning. Memory. and Cognition, 15, 657 - 668.

Blaxton, T. A. (1992). Dissociations among memory measures in memory impaired subjects: Evidence for a processing account of memory. Memory and Cognition, 20, 549 - 562.

Blaxton, T. A. (1995). A process based view of memory. Journal of International Neuropsychological Society, 1, 112 - 114.

Bowers, G. H.(1981). Mood and memory. American Psychologist, 36, 129 - 148.

Bowers, J. S., \& Schacter, D. L. (1990). Implicit memory and test awareness. Journal of Experimental Psychology: Learning, Memory, and Cognition, 16, 404 - 416.

Bradley, B. P., Mogg, K., \& Millar, N. (1996). Implicit memory bias in clinical and non-clinical depression. Behaviour Research and Therapy, 34, $865-879$.

Bradley, B. P., Mogg, K., \& Williams, R. (1996). Implicit and explicit memory for emotion-congruent information in clinical depression and anxiety. Behaviour Research and Therapy, 33, 755 - 770.

Brand, N., \& Jolles, J. (1987). Information processing in depression and anxiety. Psychological Medicine, 17, 145 - 153.

Brown, W.P., \& Ure, D. M. J. (1969). Five rated characteristics of 650 word association stimuli. British Journal of Psychology, 60, $233-249$.

Burke, D. M., \& Light, L. L. (1981) . Memory and aging: The role of retrieval processes. Psychological Bulletin, 90, 513 - 546.

Bumberry, W., Oliver, J. M., \& McClure, J. N. (1978). Validation of the beck depression inventory in a university population using psychiatric estimate as the criterion. Journal of Consulting and Clinical Psychology, 46, 150 - 155.

Butters, N., Heindel, W. C., \& Salmon, D. P. (1990). Dissociation of implicit memory in dementia: Neurological implications. Bulletin of the Psychonomic Society, 28, 359 - 366.

Calesimo, G. A. (1994). Perceptual and conceptual priming in amnesic and alcoholic patients. 
Neuropsychologia, 32, $903-921$.

Cermak, L. S., Talbot, N., Chandler, K., \& Wolbarst, L. R. (1985). The perceptual priming phenomenon in amnesia. Neuropsychologia, 23, $615-622$.

Cermak, L. S., Verfaellie, M., \& Chase, K. A. (1995). Implicit and explicit memory in amnesia: an analysis of data driven and conceptually driven processes. Neuropsychology, 9, 281 - 290.

Cermak, L. S., Verfaellie, M., \& Letourneau, L. (1993). Episodic effects on picture identification for alcoholic Korsakoff patients. Neuropsychology. 9, 281 - 290.

Challis, B. H., \& Brodbeck, D. R. (1992). Levels of processing affects priming in word fragment completion. Journal of Experimental Psychology: Learning. Memory, and Cognition, 18, 595 607.

Clark, D. M., \& Teasdale, J. D. (1985). Constraints on the effects of mood on memory. Journal of Personality and Social Psychology, 48, 1595 - 1608.

Cohen, N. J., \& Squire, L. R. (1980). Preserved Learning and retention pattern-analyzing skill in amnesia: Dissociation of knowing how and knowing that. Science, 210, 207 - 210.

Coyne, J. C. (1994). Self-reported distress: Analog or ersatz depression? Psychological Bulletin. 116, $29-45$.

Danion, J. M., Willard-Schroeder, D., Zimmerman, M. A., Grange, D., Schlienger, J. L., \& Singer, L. (1991). Explicit memory and repetition priming in depression. Archives of General Psychiatry, 48, $707-711$.

Deardoff, W. W., \& Funabiki, D. (1985). A diagnostic caution in screening for depressed college students. Cognitive Therapy and Research, 9, 277 - 284.

Denny, E. B., \& Hunt, R. R. (1992). Affective valence and memory in depression: Dissociation of recall and fragment completion. Journal of Abnormal Psychology. 101, $575-580$.

Depue, R. A., Krauss, S., Spoont, M. R., \& Arbisi, P. (1989). General behaviour inventory identification of unipolar and bipolar affective conditions in a non clinical university population. Journal of Abnormal Psychology, 98, 117 - 126.

Depue, R. A., \& Monroe, S. M. (1978). Learned helplessness in the perspective of the depressive disorders: conceptual and definitional issues. Journal of Abnormal Psychology, 87. 3-20.

Duchek, J. M., \& Heely, J. H. (1989). A dissociative word frequency times levels of processing interaction in episodic recognition and lexical decision tasks. Memory and Cognition. 17, 148 162.

Ellis, H. C. (1985). On the importance of mood intensity and encoding demands in memory: Commentary on Hasher, Rose, Zacks, Sanft \& Doren. Journal of Experimental Psychology: General. 114, 392 - 395.

Ellis, H. C. (1990). Depressive deficits in memory: Processing initiative and resource allocation. Journal of Experimental Psychology: General, 119, 60 - 62.

Elliot, C. L., \& Greene, R. L. (1992). Clinical depression and implicit memory. Journal of Abnormal Psychology, 101, $572-574$.

Ellis, H. C., \& Ashbrook, P. W. (1988). Resource allocation model of the effects of depressed mood states on memory. In K. Fielder, \& J Forgas (Eds.), Affect, cognition and social behaviour: New evidence and integrative attempts (pp. 25 - 43). Lewiston, NY: Hogrefe.

Ellis, H. C., Thomas, R. L., \& Rodriguez, I. A. (1984). Emotional mood states and memory: Elaborative encoding, semantic processing and cognitive effort. Journal of Experimental Psychology: Learning. Memory, and Cognition. 10, 470 - 482.

Fleischman, D. A., Gabrieli, J. D. E., Reminger, S., Rinaldi, J., Morrell, F., \& Wilson. (1995). 
Conceptual priming in perceptual identification for patients with alzheimers disease and a patient with right occipital lobectomy. Neuropsychology. 9, 187 - 197.

Gabrielli, J. D. E. (1995). A systematic view of human memory processes. Journal of International Neuropsychological Society, 1, 115 - 118.

Gilhooly, K. J., \& Johnson, C. E. (1978). Effects of solution word attributes on anagram difficulty: A regression analysis. Quarterly Journal of Experimental Psychology, 30, 57 - 70.

Gotlib, I. H. (1984). Depression and general psychopathology in university students. Journal of Abnormal Psychology, 93, 20 - 30.

Graf, P. G., \& Gallie, K. A. (1992). Transfer appropriate processing account for memory and amnesia. In L. Squires, \& N. Butters (Eds.), Neuropsychology of Memory (pp. 241 - 248). New York, Guilford Press.

Graf, P., \& Mandler, G. (1984). Activation makes words more accessible, but not necessarily more retrievable. Journal of Verbal Learning and Verbal Behaviour, 23, 553 - 568.

Graf, P., \& Masson, M. E. J. (Eds.) (1993). Implicit memory: New directions in cognition, development and neuropsychology. Hillsdale, NJ: Erlbaum.

Graf, P., \& Ryan, L. (1990). Transfer appropriate processing for implicit and explicit memory. Journal of Experimental Psychology: Learning, Memory, and Cognition,16,978 - 992.

Graf, P. \& Schacter, D. L. (1985). Implicit and explicit memory for new associations in normal and amnesic subjects. Journal of Experimental Psychology: Learning. Memory, and Cognition, 11. $501-518$.

Graf, P., \& Schacter, D. L. (1989). Unitization and grouping mediate dissociations in memory for new associations. Journal of Experimental Psychology: Learning. Memory, and Cognition, 15. 930 - 940 .

Graf, P., Squire, L. R., \& Mandler, G. (1984). The information that amnesic patients do not forget. Journal of Experimental Psychology: Learning. Memory, and Cognition, 10, 164 - 178.

Hammen, C. L. (1980). Depression in college students: Beyond the Beck Depression Inventory. Journal of Consulting and Clinical Psychology, 48, 126 - 128.

Hartlage, S., Alloy, L. B., Vasquez, C., \& Dykman, B. (1993). Automatic and effortful processing in depression. Psychological Bulletin, 113, $247-278$.

Hatzenbuehler, L. C., Prapal, M., \& Matthews, L. (1983). Classifying college students as depressed or nondepressed using the beck depression inventory: An empirical analysis. Journal of Consulting and Clinical Psychology, 51, 360 - 366.

Hasher, L., Rose, K. C., Zacks, R. T., Sanft, H., \& Doren, B. (1985). Mood, recall and selectivity effects in normal college students. Journal of Experimental Psychology: General, 114, 104 118.

Hasher, L., \& Zack, R. T. (1988). Automatic and effortful processes in memory. Journal of Experimental Psychology: General, 119,356 - 388.

Hastroudi, S., Parker, E. S., De Lisi, L. E., Wyatt, R. J., \& Mutter, S. A. (1984). Intact retention in acute alcohol amnesia. Journal of Experimental Psychology: Learning. Memory, and Cognition, 10, $156-163$.

Hayman, C. A. G., \& Tulving, E. (1989). Contingent dissociation between recognition and fragment completion: The method of triangulation. Journal of Experimental Psychology: Learning. Memory, and Cognition, 15,228 - 240.

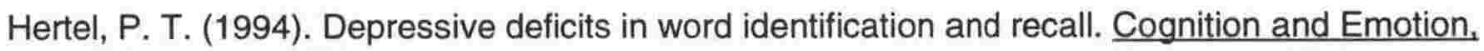
$1994,8,313-327$.

Hertel, P. T. (1994). Depression and memory: Are impairments remediable through attentional 
control. Current Directions in Psychological Science, 3. 190 - 193.

Hertel, P. T., \& Hardin, T. S. (1990). Remembering with and without awareness in a depressed mood: Evidence of deficits in initiative. Journal of Experimental Psychology: General, 119. 45 59.

Hertel, P. T. \& Knoedler, A. J. (1996). Solving problems by analogy: The benefits and detriments of hints and depressed moods. Memory and Cognition, 24, 16 - 25.

Hertel, P. T., \& Milan, S. (1994). Depressive deficits in recognition: dissociation of recollection and familiarity. Journal of Abnormal Psychology, 103, 736 - 742.

Hertel, P. T. \& Rude, S. S. (1991). Depressive deficits in memory: Focusing attention improves subsequent recall. Journal of Experimental Psychology: General. 120, 301 - 309.

Hirshman, E., Snodgrass, J. G., Mindes, J., \& Feenan, K. (1990). Conceptual priming in fragment completion. Journal of Experimental Psychology: Learning. Memory, and Cognition. 16. $634-647$.

Howard, D. V. (1991). Implicit memory: an expanding picture of cognitive aging. Annual review of gerontology and geriatrics, $11,1-22$.

Isen, A. M. (1985). Asymmetry of happiness and sadness in effects on memory in normal college students: Comment on Hasher, Rose, Zacks, Sanft, and Doren. Journal of Experimental Psychology: General, 114, 388 - 391.

Jacoby, L. L. (1991). A process dissociation framework: Separating automatic from intentional uses of memory. Journal of Memory and Language. 30, 513 - 541.

Jacoby, L. L. (1983). Remembering the data: Analyzing interactive processes in reading. Journal of Verbal Learning and Verbal Behaviour, 22, 485 - 508.

Jacoby, L. L. \& Dallas, M. (1981). On the relationship between autobiographical memory and perceptual learning. Journal of Experimental Psychology: General, 110, 306 - 340.

Java, R. I., \& Gardiner, J. M. (1991). Priming and aging: further evidence of preserved memory function. American Journal of Psychology, 104, 89 - 100.

Keane, M. M., Gabrieli, J. D. E., Fennema, A. C., Growdon, J. H., \& Corkin, S. (1991). Evidence for a dissociation between perceptual and conceptual priming in alzheimers disease. Behavioural Neuroscience, 105, 326 - 342.

Kolers, P. A. (1975). Memorial consequences of automatized encoding. Journal of Experimental Psychology: Human Learning and Memory, 1, 689 - 701.

Kolers, P. A. (1979). A pattern analyzing basis of recognition. In L. S. Cermak \& F. I. M. Craik (Eds.), Levels of Processing in Human Memory (pp. 363 - 384). Hillsdale, NJ: Erlbaum.

Kuhl, J., \& Helle, P. (1986). Motivational and volitional determinants of depression: The degenerated-intention hypothesis. Journal of Abnormal Psychology, 95, 247 - 251.

Light, L. L., \& Albertson, S. A. (1989). Direct and indirect tests of memory for category exemplars in young and older adults. Psychology and Aging, 4, 487 - 492.

Light, L. L., \& Singh, A. (1987). Implicit and explicit memory in young and older adults. Journal of Experimental Psychology: Learning. Memory, and Cognition, 13,531 - 541.

Maki, P. M. (1995). Is implicit memory preserved in alzheimers disease? Implications for theories of implicit memory. Aging and Cognition, 2, 192 - 205.

Mandler, G. (1980). Recognizing: The judgement of previous occurrence. Psychological Review, 87. $252-271$.

Masson, M. E. J., \& MacLeod, C. M. (1992). Re-enacting the route to interpretation: Enhanced perceptual identification without prior perception. Journal of Experimental Psychology: 
General, 121, $145-176$.

Mayer, D. D., \& Bower, G. H. (1985). Naturally occurring mood and learning: Comment on Hasher, Rose, Zacks, Sanft, and Doren. Journal of Experimental Psychology: General, 114, 396 - 403.

McDermott, K. B., \& Roediger, H. L. (1994). Effects of imagery on perceptual implicit memory 1390.

tests. Journal of Experimental Psychology: Learning. Memory, and Cognition, 20, 1379 -

Mendelsohn, G. A., \& O'brien, A. T. (1974). The solution of anagrams: A re-examining of the effects of transition letter probabilities, letter moves, and word frequency on anagram difficulty. Memory and Cognition, 2, 566 - 574.

Morris, C. D., Bandsford, J. D., \& Franks, J. J. (1977). Levels of processing versus transfer appropriate processing. Journal of Verbal Learning and Behaviour, 16, 519 - 533.

Naito, M. (1990). Repetition priming in children and adults: age related dissociation between implicit and explicit memory. Journal of Experimental Child Psychology, 50, 462 - 484.

Oliver, J. M., \& Simmons, M. E. (1984). Depression as measured by the DSM-III and the beck depression inventory in a unselected adult population. Journal of Consulting and Clinical Psychology, 52, $892-898$.

Perruchet, P., \& Baveuz, P. (1989). Correlational analyses of explicit and implicit memory performance. Memory and Cognition, 17, 77 - 86.

Potts, R., Camp, C., \& Coyne, C. (1989). The relationship between naturally occurring dysphoric moods, elaborative encoding and recall performance. Cognition and Emotion. 3. 197 - 205.

Radloff, L. S. (1977). The CES-D Scale: A self-report depression scale for research in the general population. Applied Psychological Measurement. 1, 385 - 401.

Rajaram, S., \& Roediger, H. L. (1993). Direct comparison of four implicit memory tests. Journal of Experimental Psychology: Learning. Memory, and Cognition. 19.765 - 776.

Rappold, V. A., \& Hashtroudi, S. (1991). Does organisation improve priming? Journal of Experimental Psychology: Learning. Memory, and Cognition,17, 103 - 114.

Rathus, J. H., Reber, A. S., Manza, L., \& Kuishner, M. (1994). Implicit and explicit learning: Differential effects of affective states. Perceptual and Motor Skills. 79, $163-184$.

Richards, P. M., \& Ruff, R. M. (1989). Motivational effects on neuropsychological functioning: Comparison of depressed versus nondepressed individuals. Journal of Consulting and Clinical Psychology, 57, $396-402$.

Richardson-Klavehn, A., \& Bjork, R. A. (1988). Measures of memory. Annual Review of Psychology, 39, 475 - 543.

Roediger, H. L. (1990). Implicit memory: A commentary. Bulletin of the Psychonomic Society, 28. $373-380$.

Roediger, H. L. (1990). Implicit memory: Intention without remembering. American Psychologist. 45. $1043-1056$.

Roediger, H. L., \& Blaxton, T. A. (1987). Effects of varying modality, surface features, and retention interval on priming in word-fragment completion. Memory and Cognition, 15, 379 388.

Roediger, H. L., \& McDermott, K. B. (1992). Depression and implicit memory: A commentary. Journal of Abnormal Psychology, 101, 587 - 591.

Roediger, H. L. \& McDermott, K. B. (1993). Implicit memory in normal human subjects. In F. Boller \& J. Grafman (Eds.), Handbook of neuropsychology, Vol. 8 (pp. 133 - 173). Amsterdam: Elsevier. 
Roediger, H. L., Rajaram, S., \& Srnivas, K. (1990). Specifying criteria for postulating memory systems. Annals of the New York Academy of Sciences, 608, 572 - 595.

Roediger, H. L., Weldon, M. S., \& Challis, B. H. (1989). Between implicit and explicit measures of retention: a processing account. In H. L. Roediger, \& F. I. M. Craik (Eds.), Varieties of memory and consciousness: Essays in honour of Endel Tulving (pp. $3-41$ ). Washington, DC: Laurence Erlbaum and Associates.

Roediger, H. L., Weldon, M. S., Stadler, M. L., \& Riegler, G. L. (1992). Direct comparison of two implicit memory tests: Word fragment and word stem completion. Journal of Experimental Psychology: Learning Memory and Cognition, 18, 1251 - 1269.

Roy-Byrne, P. P., Weingarter, H., Bierer, L. M., Thompson, K., \& Post, R. M. (1986). Effortful and automatic cognitive processes in depression. Archives of General Psychiatry, 43, 265 267.

Ruiz-Caballero, J. A., \& Gonzalez, P. (1994). Implicit and explicit memory bias in depressed and nondepressed subjects. Cognition and Emotion, 8, 555 - 569.

Schacter, D. L. (1985). Multiple forms of memory in humans and animals. In N. Weinberger, G. Lynth, \& J McGaugh (Eds.), Memory systems of the brain (pp. 351 - 379). New York: Guilford.

Schacter, D. L. (1987). Implicit memory: History and current status. Journal of Experimental Psychology: Learning, Memory, and Cognition, 19,501- 518.

Schacter, D. L. (1990). Introduction to implicit memory: Multiple perspectives. Bulletin of the Psychonomic Society, 28, $338-340$.

Schacter, D. L. (1992). Understanding implicit memory. American Psychologist, 47, 559 - 569.

Schacter, D. L. (1994). Priming and multiple memory systems. In D. L. Schacter \& E. Tulving (Eds.), Memory Systems (pp. 253 - 262). Cambridge, Mas: MIT Press.

Schacter, D. L., Bowers, J., \& Booker, J. (1989). Intention, awareness and implicit memory: The Retrieval Intentionality Criterion. In S. Lewandosky, J. C. Dunn, \& K. Kirsner (Eds.). Implicit memory. Theoretical Issues. (pp. 47 - 65). Hillsdale, NJ: Erlbaum.

Schacter, D. L., \& Graf, P. (1986). Modality specificity of implicit memory for new associations. Journal of Experimental Psychology: Learning. Memory, and Cognition, 15, 3 - 12.

Schacter, D. L., \& Graf, E. (1987). Memory Systems. Cambridge, Mas: MIT Press.

Schacter, D. L., \& Tulving, E. (1994). What are the memory systems of 1994 ? Cambridge, MIT Press.

Schwartz, B. L. (1989). Effects of generation on indirect measures of memory. Journal of Experimental Psychology: Learning. Memory, and Cognition,15, 1119 - 1128.

Schwartz, B. L., Rosse, R. B., \& Deutsch, S. I. (1993). Limits of the processing view in accounting for dissociations among memory measures in a clinical population. Memory and Cognition, 21, $63-72$.

Simamura, A. P., Squire, L. R. (1984). Paired Associate Learning and priming effects in amnesia: a neuropsychological study. Journal of Experimental Psychology: General, 113, 556 - 570.

Squire, L. R. (1986). Mechanisms of memory. Science, 232, 1612 - 1619.

Squire, L.R., Shimamura, A.P., \& Graf, P. (1987). Strength and duration of priming effects in normal subjects and amnesic subjects. Neuropsychologia, 25, $195-210$.

Stone, A. A., \& Neale, J. M. (1984). New measures of daily coping: Development and preliminary results. Journal of Personality and Social Psychology, 46, 892 - 906.

Strober, M., Green, J., \& Carlson. (1981). Utility of the beck depression inventory with psychiatrically hospitalised adolescents. Journal of Consulting and Clinical Psychology, 49. 
$482-483$

Srinivas, K., \& Roediger, H. L. (1990). Classifying implicit memory tests: Category association and anagram solution. Journal of Memory and Language, 29, $389-412$.

Tharpar, A., \& Greene, R. L. (1994). Effects of level of processing on implicit and explicit tasks. Journal of Experimental Psychology: Learning. Memory, and Cognition, 18. 671 - 679.

Toth, J. P., \& Hunt, R.R. (1990). Effects of generation on a word identification task. Journal of Experimental Psychology: Learning. Memory, and Cognition.16,993 - 1003.

Toth, J. P., Lindsay, D. S., \& Jacoby, L. L. (1992). Awareness, automaticity, and memory dissociations. In L. Squires, \& N. Butters (Eds.), Neuropsychology of Memory (pp. 241 - 248).

New York, Guilford Press.

Toth, J. P., Reingold, E. M., \& Jacoby, L. L. (1994). Towards a redefinition of implicit memory: Process dissociations following elaborative processing and self generation. Journal of Experimental Psychology: Learning. Memory, and Cognition, 20,290 - 303.

Tulving, E. (1972). Episodic and semantic memory. In E. Tulving \& W. Donaldson, (Eds.), Organization and Memory. (pp. 381 - 403). New York: Academic Press.

Tulving, E. (1983). Elements of Episodic Memory. New York: Oxford University Press.

Tulving, E., Hayman, C. A. G., \& McDonald, C. (1996). Long lasting perceptual priming and semantic learning in amnesia: a case experiment. Journal of Experimental Psychology: Learning. Memory, and Cognition, 17, 595 - 617.

Tulving, E., \& Schacter, D. L. (1990). Priming and human memory systems. Science, 247, 301 306.

Tulving, E. \& Watkins, M. J. (1973). Continuity between recall and recognition. American Journal of Psychology, 86, 739 - 748.

Vaidya, C. J., Gabrieli, J. D. E., Keane, M. M., \& Monti, L. A. (1995). Perceptual and conceptual memory processes in global amnesia. Neuropsychology, 9. $580-591$.

Verfaellie, M., \& Treadwell, J. R. (1993). Status of recognition memory in amnesia. Neuropsychology, 7, $5-13$.

Vrendenburg, K., Flett, G. L., \& Krames, L. (1993). Analogue versus clinical depression: a critical reappraisal. Psychological Bulletin, 113, 327 - 344.

Warren, M. W., \& Thomson, W. J. (1969). Anagram solution as a function of transition probabilities and solution word frequency. Psychonomic Science, 17, 333 - 334.

Warrington, K. K., \& Weiskrantz, L. (1968). A new method for testing long term retention with special reference to amnesic patients. Nature, 217, 972 - 974.

Warrington, K. K., \& Weiskrantz, L. (1970). The amnesic syndrome: Consolidation or retrieval? Nature, 228, $628-630$.

Watkins, P. C., Mathews, A., Williamson, D. A., \& Fuller, R. D. (1992). Mood congruent memory in depression: Emotional priming or elaboration. Journal of Abnormal Psychology. 101, 581 586.

Watkins, P. C., Vache, K., Vernay, S. P., Muller, S., \& Mathews, A. (1996). Unconscious moodcongruent memory bias in depression. Journal of Abnormal Psychology, 105, 34 - 41.

Watts, F. N., \& Sharrock, R. (1987). Cued recall in depression. British Journal of Clinical Psychology, 26, 149 - 150.

Weingartner, H., Cohen, R. M., Murphy, D. L., Martello, J., \& Gerdt, C. (1981). Cognitive processes in depression. Archives of General Psychiatry, 38, 42 - 47. 
Weiskrantz, L., \& Warrington, E. K. (1979). Conditioning in amnesic patients. Neuropsychologia. 17. $187-194$.

Weldon, M.S. (1991). Mechanisms underlying priming on perceptual tests. Journal of Experimental Psychology: Learning. Memory, and Cognition, 17, 526 - 541. 


\subsection{Appendix}

\section{Experiment 1}

\section{Generate sentences by list and block}

\section{List $\mathrm{A}$}

\section{Block 1 (A)}

toaster
denim
juggler
calcium
envelope
bucket
Greek
hearing
envy
fleas
computer
Charles
rake
badminton
perfume
candle

Block 2 (A)

anchor

stop

glass

reflector

Argentina

clock

torch

vinegar

attic

area

belt

private

puzzles

thunder

Helen

forceps

spear

pumpkin

Block 3 (A) one uses this to make toast $t$

jeans are made of this type of material $\mathrm{d}$

this person is able to toss and catch many balls at once $j$

an important building block for bones found in diary products $\mathrm{C}$

one sends a letter in this $e$

often used with a mop this is used to hold water b

In Greece people speak $\mathrm{G}$

the ability to hear is called $\mathrm{h}$

similar to jealousy, this is when you are desiring something someone else has e

dogs often have ticks and $f$

an Apple Mac and IBM pc are examples of a c

the name of the Queens first son and heir to the throne $\mathrm{C}$

in the autumn you clear leaves with a $r$

a game played with a racquet and shuttlecock b_

another word for cologne is $p$

this has a wick and is made of wax $\mathrm{C}$

this object is thrown overboard to become lodged in the sea bottom to one from drifting away a

used in a window to see through and keep the cold out $\mathrm{g}_{\text {. }}$

bikes should have these shiny panels to help people see them at night $r$

in the early 1980 's Britain went to war with this country over some islands A this is used to tell the time $\mathrm{C}$

usually powered by batteries one uses this at night to see $t$ some people sprinkle this onto fish and chips $v$

in some houses the area beneath the house is called a cellar while the below the roof is called an a

a cord that goes around ones waist to hold up trousers b_

the lowest position in the armed forces $p$

children often like to play with jigsaw $p$

lightning often occurs with $t$

the first name of the leader of the NZ Labour party $\mathrm{H}$

in helping to deliver a baby a doctor sometimes uses $f$

one would throw a javelin just as a primitive hunter would throw a $\mathrm{s}$

a vege with orange flesh and lots of seeds $p$

a popular cooked breakfast would be eggs and $b$

a tree that often looks bent over is a weeping $\mathrm{w}$

the people who occupy the most populated land on earth are the $C_{-}$ this is used to take photos $\mathrm{C}$

in a marriage, according to convention the male partner is called a $\mathrm{h}$ very similar to a tortoise, this animal prefers to swim in the water $t$ a nun is often referred to as a s.

Eskimos used to live in these structures made of ice i sometimes controversial this is put in town water supplies to reduce decay $f$ 
hockey

tractor

Uranus

organ

dolphin

Rome

chopstick

List B

Block 1 (B)

Caribbean

as

slacks

stool

heroin

kerosene

Egypt

brunch

glacier

taxi

to a

muffler

brass

subject

would

coconut

blanket

nurse

elephant

Block 2 (B)

a winter game played with a stick and ball $h$

on the farm a farmer sometimes uses a large vehicle called a $t$

in the solar system Saturn is close to $U$

a church choir is often accompanied by someone playing the 0

the most intelligent sea creature $\mathrm{d}$

the headquarters of the Catholic church is in R

many Asians use these to eat with $\mathrm{c}$

a sea found between north and south America that includes islands such

Barbados and Jamaica C

a name for trousers that women wear s

a type of seat with no back and often used at a bar is a s_

a white powder that if injected makes you high $\mathrm{h}$

a fuel used for heaters and camp stoves $k$

the Pharaohs built the pyramids in $E$

a name for both breakfast and lunch $\bar{b}$

Fox and Franz Joseph are names for this large cold structure $\mathrm{g}$

one can phone for this vehicle and pay the metered fare when one gets

destination $t$

used as part of an exhaust pipe to reduce noise $\mathrm{m}$

trumpets and saxophones are often made of $b$

in a sentence such as "the dog chased the cat", the phrase "the dog"

be the $\mathrm{s}$

often found on desert islands this round object is eaten for its flesh and it also contains milk c

a name for a warm object used in making $a$ bed $b$

a doctor often works with a $n$

a large grey beast with a long trunk e

surfing
raft
journal
water
banana
ballet
sapphire
Cupid

monastery

paddle

Buddhist

champagne

Darwin

clogs

hair

ashtray

Block 3 (B)

lemonade

galley

Hawaii

piranha

geology

form

strap

thumb

floor

eagle one does this on a board on a wave s

sea disaster survivors can be found floating on a $r$

a periodical that contains technical articles is called $a j$

hydroelectricity is generated using $\mathrm{w}$

this has yellow skin that one has to peel before eating $b$

Swan Lake and the Nutcracker are examples of this b_

after a diamond this is the next hardest stone $\mathrm{s}$

according to folklore this little person fires arrows to help people fall in love C

this is a place where monks or nuns live $m$

a dragon boat racer or canoeist uses this to propel through the water $\mathrm{p}$ a name for those who follow the teachings of Buddha $B$

a drink for special occasions, motor racers like to fizz this when they win a race $\mathrm{C}$

this person wrote the origin of the species $D$

traditionally Dutch people where these wooden objects $\mathrm{C}$

bald men lack this $h$

a small round object used to collect ash a

a fizzy drink containing lemon juice I_

a name for the place where the crew prepare meals in a airliner $\mathrm{g}$

a Pacific island known for large hotels, surf and beaches $\mathrm{H}$

this lives in South American waterways and has a fearsome reputation for eating flesh $p$

apart from geography another discipline which studies the earth and its

is called $\mathrm{g}$

this holds your watch onto your arm s_

the fattest finger $t$

this is what one walks on inside $f$

a emblem of the Unites States, this flies and catches small animals e_ 
ukulele

compass

used

pink

malaria

decade

intestine

October a tiny guitar $u$

often used with a map this object is sensitive to magnetic waves and is to find a direction $\mathrm{c}$

people used to dress boys in blue and girls in $p$

in the hotter parts of the world a sickness carried by fleas is called $\mathrm{m}$ every ten years is called a $d$

in this long tube like structure food is absorbed and waste collected for disposal i

Octoberfast, the German drinking festival is held at this time of year $\mathrm{O}$

Graphemic and semantic cues, word fragments and category association cues

Blocks 1 to 6

LIST A

Block 1 (A)

toaster

denim

juggler

calcium

envelope

bucket

Greek

hearing

envy

fleas

computer

Charles

rake

badminton

perfume

candle

Block 2 (A)

anchor

glass

reflector

Argentina

clock

torch

vinegar

attic

belt

private

puzzles

thunder

Helen

forceps

spear

pumpkin

\section{Graphemic}

roaster

venim

juggins

calcine

envelop

buckle

geek

heading

envoy

pleas

commuter

charges

rate

beadsman

perform

candy
Semantic

griller

corduroy

clown

potash

stationery

pail (object)

French

seeing

anguish

mosquitoes

processor

Andrew

hoe

tennis

scent

incense
Word fragments

osa_t_r
en_m
j_g_I_r
c_l__um

-nvv ${ }_{-}$op

- - ket

_re_k

e_r_ _ng

e_v

f_ea_

c _ pu_ _-

c_a_les

$r_{-}$e

b_dm_to

$p_{-} f_{-} e$

c_nd_e

\section{Category assoc}

found in a kitchen

type of cloth

part of a circus

mineral

office supplies

cleaning equipment

languages

senses

emotions

insects

scientific instrument

boys name

tools

indoor sport

cosmetics

religious article

Block 3 (A)
bacon
willow
Chinese
camera
husband
turtle
sister

anchovy
gloss
deflector
argonauts
clack
touch
vintner
Arctic
bolt
privation
nuzzles
blunder
hele
forces
speak
pumps

\section{rudder \\ window \\ tail-light \\ Chile}

watch

flashlight

ketchup

basement

braces

sergeant

scrabble

hail

Jane

clamp

arrow

turnip nc___r
I_ss
r_- I_c_or
a_g_n_i_a

_loc

to $h$

_in_g_r

$\bar{b}_{--i}^{-i c}$

p_i_te

uz_I_

th_- er

he

- rc_ps

$\mathrm{sp}_{-}{ }_{-} \mathrm{r}$

$\mathrm{pu}_{-} \_\mathrm{k} \_\mathrm{n}$ part of a boat

building material

part of a bike

southern hemisphere

country

general electrical

appliance

camping eqiupment

food flavouring

rooms in a building

used to fasten clothing

military rank

indoor toy

weather phenomena

girls name

surgical tool

weapon

vegetable

meats

trees

ethnic group

optical instrument

relatives

reptiles

members of the

clergy 


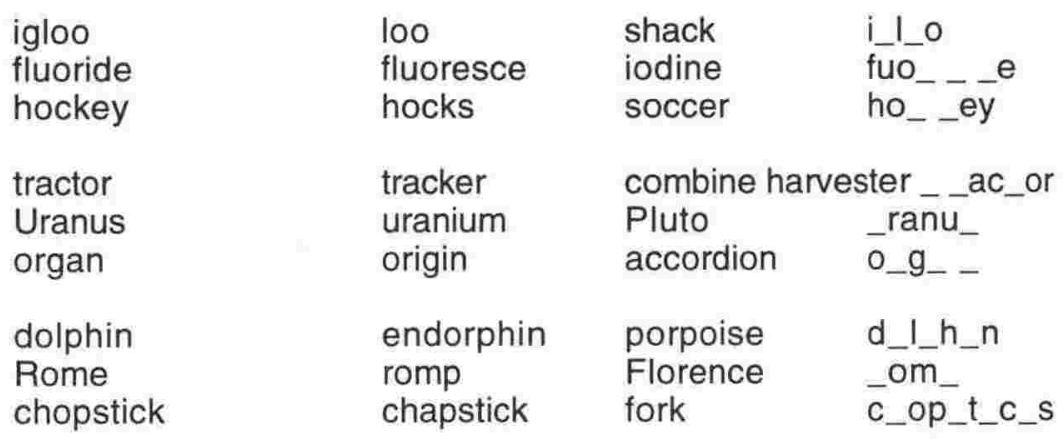

type of dwelling chemical elements sport played with a ball large vehicle planets wind musical instrument marine mammel cities eating utensils

\section{List B}

Block 1 (B)

\begin{tabular}{|c|c|c|c|}
\hline Caribbean & carib & Mediterranean & -ar__b_ ${ }_{-}$n \\
\hline slacks & slacker & skirt & sla__s \\
\hline stool & stoop & chair & too_ \\
\hline heroin & hero & cocaine & $\bar{h}_{-} r_{-} n$ \\
\hline kerosene & keratin & gasoline & k__o__ne \\
\hline Egypt & egret & Israel & $-g y_{-}-$ \\
\hline brunch & bunch & dinner & b_u_. $h$ \\
\hline glacier & glassier & iceberg & g__ci_r \\
\hline taxi & $\operatorname{maxi}$ & cab & $t_{-}$i \\
\hline muffler & muff & exhaust & m_f_l_- \\
\hline brass & bass & copper & b__s \\
\hline subject & sublet & pronoun & $s_{-}$j_ct \\
\hline coconut & cocoa & pineapple & c_c_n \\
\hline blanket & blank & bedspread & I nk_t \\
\hline nurse & purse & pharmacist & $\bar{n}_{-}$se \\
\hline elephant & element & hippopotamus & el__ha_. \\
\hline
\end{tabular}

oceans

female clothing article of furniture drug fuels northern hemisphere country meal names natural earth formation vehicle parts of a car metal parts of speech non citrus fruits part of a bed occupations 4 footed animal

Block 2 (B)

surfing
raft
journal
water
banana
ballet
sapphire
Cupid
monastery
paddle
Buddhist
champagne
Darwin
clogs
hair
ashtray

\begin{tabular}{|c|c|c|}
\hline surfeit & bodyboarding & s_r_i_g \\
\hline rift & life boat & $-\mathrm{ft}$ \\
\hline journey & periodical & - o_ nn_l \\
\hline waiter & liquid & $w_{-}-r^{r}$ \\
\hline bandanna & apple & -an_n_ \\
\hline bullet & waltz & ba_l_- \\
\hline samphire & emerald & _app_i__- \\
\hline cupric & Hercules & $\mathrm{Cu}_{-}-$ \\
\hline mastery & abbey & mon_s__r_ \\
\hline peddle & oar & pa__le \\
\hline Ghandhist & Hindu & _ _d_h_st \\
\hline campaign & wine & c_a_p_g_e \\
\hline rawin & Einstein & $d_{-} \mathrm{rw}_{-}$ \\
\hline clods & shoes & $c_{-}$gs \\
\hline here & nose & h_i_ \\
\hline astray & cigarette & _s_t_ay \\
\hline
\end{tabular}

Block 3 (B)

lemonade
galley
Hawaii
piranha
geology
strap
thumb
floor
eagle
ukulele
compass

$\begin{array}{lll}\text { marinade } & \text { Fanta } & \text { I_m___d_ } \\ \text { gallery } & \text { cockpit } & \text { g____y } \\ \text { haw } & \text { California } & \text { saw_i } \\ \text { paranoia } & \text { snapper } & \text { p___n_a } \\ \text { neology } & \text { ecology } & \text { w_o_o_y } \\ \text { strip } & \text { wristband } & \text { s_r_p } \\ \text { thump } & \text { finger } & \text { t_um_u } \\ \text { flour } & \text { ceiling } & \text { f__or } \\ \text { eager } & \text { hawk } & \text { e_g_e } \\ \text { allele } & \text { banjo } & \text { u___le } \\ \text { compare } & \text { barometer } & \text { _om_a_s }\end{array}$
watersport type of ship reading material liquid citrus fruit a type of dance percious stone mythological creature building for religious services
sports equipment religions alcholic beverages famous scientist types of footear parts of a face used by smokers

nonalcholic beverage
part of an aeroplane
US state
river fish
sciences
part of a watch
of the external body
part of a room
birds
stringed instrument
measuring device




\begin{tabular}{|c|c|c|c|c|}
\hline $\begin{array}{l}\text { pink } \\
\text { malaria } \\
\text { decade } \\
\text { intestine } \\
\text { October }\end{array}$ & $\begin{array}{l}\text { punk } \\
\text { malaise } \\
\text { decide } \\
\text { intestate } \\
\text { octonal }\end{array}$ & $\begin{array}{l}\text { yellow } \\
\text { cholera } \\
\text { century } \\
\text { guts } \\
\text { November }\end{array}$ & 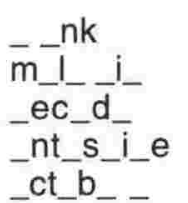 & $\begin{array}{l}\text { colours } \\
\text { diseases } \\
\text { units of time } \\
\text { organs of the body } \\
\text { months }\end{array}$ \\
\hline
\end{tabular}

\section{Experiment 2}

\section{Generate sentences by list and block}

\section{LIST A}

\section{Block 1}

lemonade

Argentina

attic

forceps

fleas

taxi

glacier

ballet

spear

perfume

muffler

private

paddle

elephant

Block 2

bacon

dolphin

clogs

anchor

Cupid

badminton

floor

Hawaii

juggler

camera

strap

ukulele

Helen

Rome

\section{Block 3}

puzzle

Carribean

geology

Uranus

envelope

coconut

pink

heroin

malaria

glass

envy

vinegar a fizzy drink containing lemon juice I_ in the early 1980 's Britain went to war with this country over some islands A in some houses the area beneath the house is called a cellar while the area below the roof is called an a

in helping to deliver a baby a doctor sometimes uses $f_{-}$ dogs can have ticks and $f$

one can phone for this vehicle and pay the metered fare when one gets to a destination t_

Fox and Franz Joseph are names for this large cold structure $g_{-}$

Swan Lake and the Nutcracker are examples of this b_

one would throw a javelin just as a primitive hunter would throw a s_ another word for cologne is $p_{-}$

used as part of a exhaust pipe to reduce noise $\mathrm{m}_{-}$

the lowest position in the armed forces $p_{-}$

a dragon boat racer or canoeist uses this to propel through the water $p_{-}$

a large grey beast with a long trunk $e_{-}$

a popular cooked breakfast would be eggs and b_

sea creature known for its intelligence and playfulness $d_{-}$

traditionally Dutch people wear these wooden objects $\mathrm{c}_{-}$

this object is thrown overboard to become lodged in the sea bottom to stop one from drifting away a

according to folklore this little person fires arrows to help people fall in love $\mathrm{C}_{-}$

a game played with a racquet and shuttlecock $b_{-}$

this is what one walks on inside $f_{\text {_ }}$

a Pacific island known for large hotels, surf and beaches $\mathrm{H}_{-}$

this person is able to toss and catch many balls at once $\mathrm{j}_{-}$

this is used to take photos $C_{-}$

this holds your watch onto your arms s_

a tiny guitar $u_{-}$

the first name of the leader of the NZ Labour party $\mathrm{H}_{-}$

the headquarters of the Catholic church is in $R_{-}$

children often like to play with jigsaw $p_{-}$

a sea found between the Southern United States and South America that includes islands such as Barbados and Cuba $\mathrm{C}_{2}$

apart from geography another discipline which studies the earth and its form is called $g_{-}$

in the solar system Saturn is close to $U_{-}$

one sends a letter in a $e_{-}$

often found growing on $\bar{a}$ desert island palm this round object is split open for its milk and flesh c_

people used to dress boys in blue and girls in $\mathrm{p}_{-}$

a white powder that if injected makes you high $\mathrm{h}_{-}$

in the hotter part of the world a sickness carried by fleas is called m

used in a window to see through and keep the cold out $\mathrm{g}_{\text {_ }}$

similar to jealousy, this is when you are desiring something someone else has $e_{-}$ some people sprinkle this onto fish and chips $v_{\text {_ }}$ 


\section{LIST B}

\section{Block 1}

chopstick

kerosene

Charles

ashtray

scales

radio

denim

blanket

eagle

brass

raft

Buddhist

belt

sister

\section{Block 2}

thunder

compass

hair

pumpkin

tractor

nurse

reflector

stool

brunch

thumb

organ

igloo

galley

hockey

\section{Block 3}

rake

bucket

handbag

candle

surfing

journal

champagne

subject

intestine

decade

Egypt

monastery

turtle

torch many Asians use these to eat with $c$

a fuel used for heaters and camp stoves $k$

the name of the Queens first son and heir to the throne $\mathrm{C}_{-}$

a small round object used to collect ash a

this is used to weigh people and other objects s_ $_{\text {. }}$

one turns this on to hear talkback $r_{\text {_ }}$

jeans are made of this material $d_{\text {_ }}$

as well as sheets one uses this in making up $a$ bed $b$

a emblem of the United States, this flies and catches small animals e_

trumpets and saxophones are often made of $b$

sea disaster survivors can be found floating on a $r$

a name for those who follow the teaching of Buddha B.

a cord that goes around one waist to hold up trousers b_

a nun is often referred to as a s.

lightning often occurs with $t$

often used with a map to find a direction, this object is sensitive to magnetic waves

c_

bald men lack this $h$

a vege with orange flesh and lots of seeds $p_{-}$

on the farm a farmer sometimes uses a large vehicle called a t

a doctor often works with a $n$

bikes often have these shiny panels to help them stand out at night $r_{-}$

a type of seat with no back and often used at a bar is a s_

a name for both breakfast and lunch $b_{-}$

the fattest finger $t$

a church choir is often played by someone playing the $0_{-}$

Eskimos used to live in these structures made of $i_{-}$

a name for a place where the crew prepare meals in a airliner $\mathrm{g}_{\text {_ }}$

a winter game played with a stick and ball $\mathrm{h}_{\text {}}$

in the autumn one clears leaves with a $r_{-}$

often used with a mop this is used to hold water b

sometimes carried by a woman over her arm to hold money and other small

objects $\mathrm{h}_{-}$

this has a wick and is made of wax c

one does this on a board on a wave s

a periodical that contains technical articles is called $a j_{-}$

a drink for special occasions, motor racers like to fizz this when they win a race $c_{-}$

in a sentence such as "the dog chased the cat", the phrase "the dog" would be

the $\mathrm{s}$

inside the human this long tube like structure digests and absorbs food $i_{-}$

every ten years is called a d

the Pharaoh's built the pyramids in $\mathrm{E}_{\text {}}$

this is a place where nuns or monks live $m$

very similar to a tortoise this small animal prefers to swim in the t_

usually powered by batteries one uses this at night to see t_ 
Category association and word fragment cues

\section{LIST A}

\section{Block 1}

lemonade

Argentina

attic

forceps

fleas

taxi

glacier

ballet

spear

perfume

muffler

private

paddle

elephant

Block 2

\section{category association}

nonalcoholic beverages

southern hemisphere countries

rooms in a building

surgical tools

insects

vehicles

natural earth formations

types of dance

weapons

cosmetics

parts of a car

military ranks

sports equipment

4 footed animals

types of meat

marine animals

types of footwear

parts of a boat

mythological creatures

indoor sports

parts of a room

Unites States state names

found in a circus

optical instruments

parts of a watch

stringed musical instruments

girls names

cities

indoor games

oceans

sciences

planets

office supplies

fruits

colours

drugs

diseases

building materials

emotions

food flavourings

names of trees

languages

\section{word fragment}

l_m__o_d
a_g_n_i_a
a__ic
f__c_ps
f_ea_-
t__i
g__ci_r
a_l_t
sp__r
e_f_me
m_f_l
p_i_te
pa__le
el__ha

b_co

d_I_h_n

c_-gs

a_c $c_{--} r$

$\mathrm{Cu}_{-}$

b_dm_ _to

f_- $r$

aw_i

j_g_I_r

$\mathrm{C}_{-}$er

s_r_p

u_ I le

he _ n

om_

p_z_l

C_r $r_{-} b_{-} n$

g _o_o_y

ranu

nvv ${ }_{-} \mathrm{op}_{-}$

c_c_n - -

- nk

h_r $n$

m- i

e-v

v_n_g_r

w_I_-w

g_e_k

\section{LIST B}

\section{Block 1}

chopstick

kerosene

Charles

ashtray

scales

radio

denim

eating utensils

fuels

boys names

used by smokers

found in a bathroom

electrical appliances

types of cloth c_op_t_c

k__o_ne

c_a_les

a_ht__y

$s_{-}$le

$r_{-}$io

_en_m 


blanket
eagle
brass
raft
monastery
belt
sister

\section{Block 2}

parts of a bed

birds

metals

types of ship

buildings for religious services

used to fasten buildings

members of the clergy b__nk_t

e_g_e

b_- ss

$--\overline{f t}$

mon_s__r_

b__t

_is_er weather phenomena

measuring devices

parts of the human face

vegetables

large vehicles

occupations

parts of a bicycle

articles of furniture

meal names

parts of the external human body

wind musical instruments

types of dwellings

found in a aeroplane

sports played with a ball th___er

om_a_s

$\overline{\mathrm{h}}$ i__

pu__k_n

_-_ac_or

n__se

r_-1_c_or

too_

b_u _

t_um_

o_g -

i_l_o

g_I__y

ho__ey

Block 3

subject
rake
bucket
handbag
candle
surfing
journal
champagne
intestine
decade
Egypt
buddhist
turtle
torch

parts of speech

tools

cleaning equipment

leather products

used in a church

watersports

reading materials

alcoholic beverages

parts of the internal human body

units of time

northern hemisphere countries

religions

reptiles

camping equipment

$$
\begin{aligned}
& \text { s__j_ct } \\
& \text { r__e } \\
& \text { b_-k_t } \\
& \text { h_n_ba_ } \\
& \text { c_nd_e } \\
& \text { s_r_i_g } \\
& \text { j_-_n_l } \\
& \text { c_a_p_g_e } \\
& \text {-nt_s_i_e } \\
& \text { ec_d_ } \\
& \text { e_y_t } \\
& \text { b__d_-st } \\
& \text { ur_le } \\
& \text { to__n }
\end{aligned}
$$

\section{Experiment 3}

Generate sentences and anagram cues by list and block

List A

\section{Block 1}

$\begin{array}{cl}\text { target } & \text { anagram } \\ \text { laugh } & \text { uhalg } \\ \text { barge } & \text { brgea } \\ \text { spend } & \text { npsde } \\ \text { chair } & \text { iharc } \\ \text { knife } & \text { fniek } \\ \text { shell } & \text { lehsl } \\ \text { brain } & \text { narib }\end{array}$

sentence

vocal sound made to express amusement or joy I

flat bottomed canal boat often used to transport coal etc $b_{-}$

act of paying out money or to use up energy s_

moveable seat which one sits on often at a desk $c_{-}$

a sharp object consisting of a one edged blade set in a handle $\mathrm{k}$

found on the beach these are collected for their shape and colours s_

soft cerebral object in the skull otherwise known as grey matter $b$ 


$\begin{array}{ll}\begin{array}{l}\text { flour } \\ \text { crown }\end{array} & \begin{array}{l}\text { fulor } \\ \text { wronc }\end{array} \\ \text { thumb } & \text { btmuh } \\ \text { spoon } & \text { onosp } \\ \text { ferry } & \text { fyrer } \\ \text { mouse } & \text { usome } \\ \text { cloud } & \text { locdu } \\ \text { rugby } & \text { bygur } \\ \text { horse } & \text { rhseo }\end{array}$

finely ground wheat etc often used in baking $f$ traditionally worn on the heads of royalty at formal occasions $\mathrm{C}_{\text {_ }}$

short thick inner digit on the human hand $t$ implement used to drink soup or eat pudding s_ this type of ship carries passengers and freight between islands $f$ common species of small rodent $\mathrm{m}_{\text {_ }}$ these float in the sky and release rain $c_{\text {_ }}$ this game has scrums and line outs and is a popular game in New Zealand $\mathrm{r}_{\text {_ }}$ an animal rode for racing and dressage $\mathrm{h}_{\text {_ }}$

Block 2

\begin{tabular}{|c|c|}
\hline $\begin{array}{l}\text { grape } \\
\text { video }\end{array}$ & $\begin{array}{l}\text { gaepr } \\
\text { idevo }\end{array}$ \\
\hline wagon & wgano \\
\hline cream & cmare \\
\hline youth & yhtuo \\
\hline ghost & gtsoh \\
\hline shirt & thisr \\
\hline bacon & bcnao \\
\hline fence & cefen \\
\hline queen & eneuq \\
\hline mould & umdlo \\
\hline rifle & lefir \\
\hline bread & dreba \\
\hline peace & cepae \\
\hline $\begin{array}{l}\text { dress } \\
\text { month }\end{array}$ & $\begin{array}{l}\text { sdsre } \\
\text { ntmho }\end{array}$ \\
\hline
\end{tabular}

this is used to make wine $\mathrm{g}_{-}$

technology which allows recording and playback of scenes $v_{-}$

wheeled vehicle for transporting heavy loads often by a team of animals $\mathrm{w}_{\text {. }}$

if you beat this long enough it turns into butter $\mathrm{c}_{-}$ a term for a younger person $y_{-}$

spirit of a dead person appearing to have a living form $\mathrm{g}_{-}$

mans garment reaching from the neck to the lower waist, often worn with a tie s_

a popular cooked breakfast would be eggs and $b_{-}$ barrier used to enclose an area often to keep animals in or out $f$

the female equivalent of a king q

object into which a substance can be placed to harden to a desired shape $\mathrm{m}_{\text {. }}$

a particular type of gun with a long barrel $r_{-}$ food made out of kneaded flour and yeast $b_{-}$ state of calm and cessation of war $p_{-}$

a woman's one piece skirted outer garment is a $\mathrm{d}_{-}$ there are 12 of these in a year $m_{-}$

\section{Block 3}

$\begin{array}{ll}\text { plane } & \text { Ipnea } \\ \begin{array}{l}\text { virus } \\ \text { judge } \\ \text { decisions j_ } \\ \text { liver }\end{array} & \text { vrsui } \\ \text { gudej } \\ \text { brass } & \text { Irvei } \\ \text { sport } & \text { srsb } \\ \text { baker } & \text { orpst } \\ \text { drill } & \text { kaerb } \\ \text { radio } & \text { lidlr } \\ \text { truck } & \text { rdoia } \\ \text { match } & \text { kutcr } \\ \text { wheel } & \text { cmtha } \\ \text { vicar } & \text { elehw } \\ \text { album } & \\ \text { noise } & \text { vrcai } \\ \text { prize } & \text { ulbma } \\ & \text { senio } \\ & \text { pizre }\end{array}$

one flies in this between destinations $p_{-}$

very small agent which can cause illness $v_{-}$ official who sits in front of a court and passes

large organ of the body which secretes bile and purifies the blood I

trumpets and saxophones are often made of $b_{-}$ general term for any activity engaged in by more than one person for fitness and pleasure $s_{-}$ person who makes cakes and breads $b_{-}$ object used to make holes in wood or teeth d_ one switches this on to hear talk-back $r_{\text {_ }}$ large vehicle for carrying heavy loads $t$ one strikes this to light a cigarette or fire $\mathrm{m}_{\text {. }}$ circular object which spins and helps a vehicle move forwards $w_{-}$ anglican term for a priest $v_{\text {_ }}$ one places stamps or photos into this a loud or unpleasant sound $\mathrm{n}_{\text {_ }}$ reward gained for often winning a competition $p_{-}$ 
List $B$

Block 1

\begin{tabular}{|c|c|}
\hline torch & troch \\
\hline cycle & ycelc \\
\hline steam & temsa \\
\hline clock & olkcc \\
\hline $\begin{array}{l}\text { blind } \\
\text { cheek }\end{array}$ & $\begin{array}{l}\text { Indib } \\
\text { ehcek }\end{array}$ \\
\hline sugar & gausr \\
\hline ankle & aekln \\
\hline naked & dneka \\
\hline razor & zrora \\
\hline earth & ehtra \\
\hline clerk & ckerl \\
\hline swing & gnsiw \\
\hline novel & Iveon \\
\hline mouth & otuhm \\
\hline ile & misle \\
\hline
\end{tabular}

usually powered by batteries one uses this at night to see t_

another shortened term for a bike c_

when water is heated it turns to $\mathrm{s}_{\text {_ }}$

this is used to tell the time $\mathrm{C}$

the state of not being able to see $b_{-}$

the side of the face below the eyes sometimes

with a rosy complexion c

white substance mixed into drinks or food to make it sweeter s_

joint connecting the leg with the foot a

to be not wearing any clothes is to be $\mathrm{n}_{-}$

sharp object used for shaving $r_{-}$

our own planet $e_{\text {_ }}$

person who works in an office to copy letters, keep files, make entries etc. $c_{-}$

playground object which one sits on and can be pushed back and forth $\mathrm{s}$

long fictional story in the form of a book $n_{\text {_ }}$

opening in the head through which one eats and speaks $\mathrm{m}$

the act of drawing the lips apart and up to show joy and amusement $\mathrm{s}_{\text {_ }}$

\section{Block 2}

\begin{tabular}{|c|c|}
\hline $\begin{array}{l}\text { nanny } \\
\text { salad }\end{array}$ & $\begin{array}{l}\text { nynna } \\
\text { sdaal }\end{array}$ \\
\hline apron & anopr \\
\hline $\begin{array}{l}\text { plate } \\
\text { bride }\end{array}$ & $\begin{array}{l}\text { tpale } \\
\text { bdrie }\end{array}$ \\
\hline chain & nhcai \\
\hline train & intar \\
\hline flash & fhals \\
\hline & $\begin{array}{l}\text { satpr } \\
\text { bygur }\end{array}$ \\
\hline paint & tinap \\
\hline fruit & trfui \\
\hline sweat & atwse \\
\hline $\begin{array}{l}\text { bible } \\
\text { magic }\end{array}$ & $\begin{array}{l}\text { libeb } \\
\text { aicma }\end{array}$ \\
\hline angel & ganel \\
\hline
\end{tabular}

a child's nurse or a she goat $\mathrm{n}_{\text {- }}$

dish often made up of lettuce, tomatoes etc. and served with a dressing s_

a cook wears this around their waist to keep their clothes clean a

round object which one eats off $p_{-}$

in a marriage the man is the groom and the woman the $\mathrm{b}_{-}$

series of metal links each passing through the next used for locking objects $c_{-}$

this runs on tracks to carry goods and passengers t.

sudden bright light $f_{-}$

this holds your watch onto your wrist $\mathrm{s}_{\text {_ }}$

this game has scrums and line outs and is a

popular game in New Zealand $r_{-}$

colouring matter mixed in a liquid and applied to a surface $\mathrm{p}_{-}$

apples, pears and bananas are examples of $f_{-}$ another name for perspiration $\mathrm{s}$

christian book of sacred writings $b$

conjuring tricks and other unexplained acts is called $\mathrm{m}$

spiritual being often portrayed with wings and harp $a_{-}$

\section{Block 3}

$\begin{array}{ll}\text { lunch } & \text { nculh } \\ \text { dance } & \text { andec } \\ \text { pilot } & \text { iltpo } \\ \text { camel } & \text { calem } \\ \text { arrow } & \text { awror } \\ \text { honey } & \text { yneoh } \\ \text { sheet } & \text { heset } \\ \text { fibre } & \text { febir }\end{array}$

a meal one has around the middle of the day I_ ballet and waltz are forms of $d_{-}$

this person flies an aeroplane $p_{-}$

a desert animal with one or more humps $\mathrm{C}_{-}$

this is fired with a bow at a target a

bees make this sweet liquid $h$

large piece of cotton etc. used in making a bed s_ thread like filament in cereals etc. which aids in digestion $\mathrm{f}_{\text {- }}$ 


$\begin{array}{ll}\begin{array}{l}\text { smell } \\ \text { nurse } \\ \text { glass }\end{array} & \begin{array}{l}\text { IsIme } \\ \text { suren } \\ \text { sgsla }\end{array} \\ \text { stool } & \text { sltoo } \\ & \\ \text { motor } & \text { romot } \\ \text { sperm } & \text { smrep } \\ \text { blood } & \text { doolb } \\ \text { skirt } & \text { ksrti }\end{array}$

sense through which one can perceive odours $\mathrm{s}_{-}$ a doctor often works with a $n$ used in a window to see through and keep the cold out g_

a type of seat with no back and often used at a bar is a s_.

simple name for an engine in a car $\mathrm{m}_{\text {_ }}$ produced by males this can fertilise an egg $\mathrm{s}_{-}$ red fluid circulating in the arteries $b$

woman's outer garment worn around the waist and covering some or most of the legs $\mathrm{s}_{-}$

\section{Experiment 4}

\section{Generate sentences and word association cues by list and block}

\section{List A}

\section{Block 1}

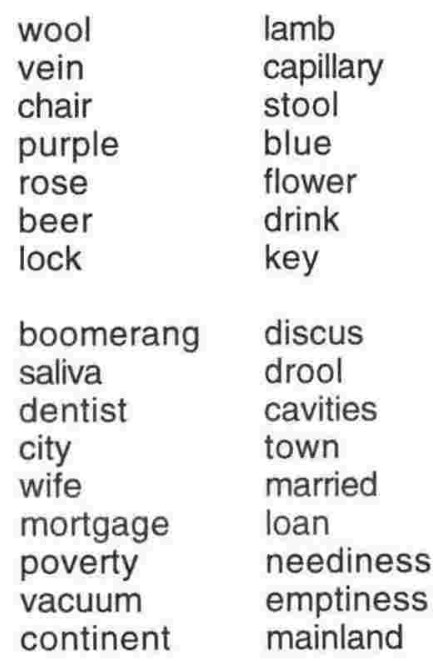

this is shorn off and spun ready for knitting $\mathrm{w}_{\text {_ }}$ these carry the blood back to the heart $v_{-}$ object used for sitting on $\mathrm{C}_{-}$ colour often associated with royalty $\mathrm{p}_{-}$ common flower with prickles on the stems $r_{-}$ a popular beverage made out of hops $b_{-}$ this is used to stop an unwanted person from opening a cupboard I

you throw this object and it is supposed to return to you b_ substance produced in the mouth to digest food $\mathrm{s}_{-}$ a person who inspects and looks after teeth d_ a large metropolitan area such as Auckland is called a c_ term used for the female partner in a marriage $\mathrm{w}_{-}$ the money owing on a property is called a $\mathrm{m}_{\text {_ }}$ what is another word for destitution. $p_{-}$ what is the term for any space devoid of matter $v_{-}$ the 5 major landmasses in the world are called c_

\section{Block 2}

$\begin{array}{ll}\text { incense } & \text { smell } \\ \text { gun } & \text { pistol } \\ \text { scales } & \text { weigh } \\ \text { marble } & \text { quartz } \\ \text { rake } & \text { spade } \\ \text { letter } & \text { writing } \\ \text { snow } & \text { sleet } \\ \text { smile } & \text { sneer } \\ \text { chimney } & \text { soot } \\ \text { hand } & \text { arm } \\ \text { blanket } & \text { bedspread } \\ \text { death } & \text { life } \\ \text { dress } & \text { blouse } \\ \text { video } & \text { film } \\ \text { barrel } & \text { cask } \\ \text { colleague } & \text { partner }\end{array}$

some people like to burn this for its aroma i_ another name for a rifle $\mathrm{g}_{-}$ object used to measure the weight of a object $\mathrm{s}_{-}$ in ancient times this stone was often used to carve out statues m_ tool used for scraping the soil and clearing up leaves $\mathrm{r}_{-}$ mail is often made up of I_ cold white substance found on the ground $s_{\text {_ }}$ a pleased expression which involves a drawing up of the lips $\mathrm{s}_{-}$ object that enables the smoke to clear from a fireplace $c_{-}$ found on the end of the arms $h_{\text {_ }}$ warm object commonly used in making up a bed b_ when a person ceases to live it is called $d$ a common name for a womans garment $d_{-}$ people hire these to watch movies at home $v_{-}$ a round wooden vessel held together by hoops is called $a b_{-}$ what do you call a professional co-worker c_

\section{Block 3}

$\begin{array}{ll}\begin{array}{l}\text { boy } \\ \text { stairs } \\ \text { leaf }\end{array} & \begin{array}{l}\text { child } \\ \text { ladder } \\ \text { tree }\end{array} \\ \begin{array}{l}\text { sugar } \\ \text { web }\end{array} & \begin{array}{l}\text { sweet } \\ \text { spider }\end{array}\end{array}$

a male human child b_ these enable one to climb up to the next level in a building $s_{-}$ found on the end of stems these gives plants their green colour I diabetics try not to eat s filmy membrane spun to trap insects $\mathrm{w}_{-}$ 


$\begin{array}{ll}\begin{array}{l}\text { thread } \\ \text { jandel }\end{array} & \begin{array}{l}\text { pin } \\ \text { sandal }\end{array} \\ \text { mountain } & \text { hill } \\ \text { washing } & \text { soap } \\ & \\ \text { street } & \text { lane } \\ \text { carrot } & \text { vegetable } \\ \text { fishing } & \text { trolling } \\ \text { deer } & \text { moose } \\ \text { computer } & \text { processor } \\ \text { gymnastics } & \text { aerobics } \\ \text { concrete } & \text { mortar }\end{array}$

Block 4

clown
butter
pepper
chicken
candle
crowd
bomb
choir
track
knee
money
summer
music
parliament
marathon
elephant

\section{List B}

\section{Block 1}

$\begin{array}{ll}\begin{array}{l}\text { laugh } \\ \text { pilot } \\ \text { pond } \\ \text { fuse } \\ \text { accountant }\end{array} & \begin{array}{l}\text { giggle } \\ \text { aeroplane } \\ \text { water } \\ \text { electricity } \\ \text { auditor }\end{array} \\ \text { anger } & \text { mad } \\ \text { apple } & \text { fruit } \\ \text { hair } & \text { head } \\ \text { sailor } & \text { soldier } \\ \text { roof } & \text { ceiling } \\ \text { park } & \text { swing } \\ \text { jeans } & \text { corduroy } \\ \text { house } & \text { cottage } \\ \text { meteor } & \text { asteroid } \\ \text { oyster } & \text { scallop } \\ \text { jungle } & \text { wilderness }\end{array}$

\section{Block 2}

$\begin{array}{ll}\text { sunrise } & \text { twilight } \\ \text { jump } & \text { leap } \\ \text { thief } & \text { criminal } \\ \text { pyramid } & \text { sphinx } \\ \text { eagle } & \text { falcon } \\ \text { butcher } & \text { shop }\end{array}$

train

elbow

bank

spring

sound

legislature

sprint this is used to sew cloth together $t$

in the summer many New Zealanders like to wear these on their feet j_

Everest is a m_

name for a activity which a person does to make themselves or objects clean w_

one drives down this $s_{-}$

popular food with a tapering orange coloured root $\mathrm{C}_{-}$

this activity uses a rod and a line f_

four footed animal often with antlers $d_{\text {_ }}$

an Apple Mac is a c_

this Olympic sport involves tumbling and floor exercises $g_{-}$

what building material is made from mixing cement and water $\mathrm{C}_{-}$

pantomine a modern version of the jester this person dresses up and likes to make people happy $\mathrm{c}_{-}$

margarine some people like to spread this on a sandwich $b$

spice some people like to shake this onto their food before eating $p_{\text {_ }}$

duck this is plucked and then often roasted $c_{-}$

lamp object with a wick which burns and glows $\mathrm{c}_{-}$

gathering a term for a largish group of people $\mathrm{C}_{-}$

explosion object used to blow things up b_

chorus a group of singers who perform in church or in public $\mathrm{c}_{-}$

a locomotive runs along these $t$

this joint enables ones leg to bend $k_{\text {_ }}$

dollars and cents are examples of $\mathrm{m}_{-}$

hottest season of the year $s_{-}$

classical, country and rock are examples of $m_{-}$

hippopotamus large grey beast with a large trunk $e_{-}$

New Zealand's house of representatives is called $p_{-}$

noise made when one is happy I_

this person flies a aeroplane $p_{-}$

a small body of water found in a garden often with gold fish $p_{-}$

when this blows the power goes out $f$

the name for a person who manages the finances in a

organisation $\mathrm{a}_{-}$

a general term for when a person becomes enraged and shouts a

according to folklore one of these a day will keep the doctor away a

people often need to shampoo this $h_{-}$

a general term for a sea man in the navy below the rank of an

officer $\mathrm{s}$

covering over the top of a building $r_{-}$

public garden often with a play area is called a $p_{-}$

a popular form of trousers $j_{L}$

a general term for a dwelling $h_{\text {_ }}$

what causes large holes in the earth called star wounds $\mathrm{m}_{-}$

what seafood delicacy is grown in beds o.

what is an area of tropical vegetation called $\mathrm{j}_{-}$

the time of day when the son comes up in the morning is called s_ to hop up and down with a muscular movement of the legs is to j_ a name for a person who steals things $t$

the pharaohs built these large triangular objects $p_{-}$

an emblem of the United States this flies and catches mice $e_{-}$ name for a person who prepares and sells meat $b_{\text {_ }}$ 


\begin{tabular}{|c|c|c|}
\hline $\begin{array}{l}\text { diving } \\
\text { light }\end{array}$ & $\begin{array}{l}\text { swimming } \\
\text { dark }\end{array}$ & $\begin{array}{l}\text { leaping off a springboard into the water below is called } d_{-} \\
\text {this enables one to see I_ }\end{array}$ \\
\hline jury & court & group of people who decide whether a person is guilty or not j_ \\
\hline tea & cocoa & popular beverage made out of dried leaves $t$ \\
\hline God & heaven & general name for a supreme being g_ \\
\hline dinner & meal & the occasion of taking food in the evening is called d_ \\
\hline lion & panther & large African animal in the cat family I_ \\
\hline universe & cosmos & what is the big bang said to have created $u_{-}$ \\
\hline victory & success & $\begin{array}{l}\text { what was Winston Churchill referring to when he gestured with } 2 \\
\text { fingers in the air v_ }\end{array}$ \\
\hline chitect & designer & what type of professional draws up plans for buildings a \\
\hline
\end{tabular}

Block 3

\begin{tabular}{|c|c|c|}
\hline clock & watch & this is used to tell the time $c_{-}$ \\
\hline lawn & grass & a patch of flat land that is mown I_ \\
\hline bat & ball & one swings this in a softball game $b_{-}$ \\
\hline man & bloke & a grown up male $\mathrm{m}_{-}$ \\
\hline knob & handle & $\begin{array}{l}\text { a rounded lump on the surface of something which has to be } \\
\text { turned to open it k_ }\end{array}$ \\
\hline mirage & delusion & $\begin{array}{l}\text { in hot countries the effect of seeing water that is not there far off } \\
\text { in the distance is called a m_ }\end{array}$ \\
\hline monkey & orangutan & a non human primate $\mathrm{m}_{-}$ \\
\hline mouse & rat & a small rodent $\mathrm{m}_{-}$ \\
\hline swamp & bog & a boggy wet piece of land is called a s_ \\
\hline oxygen & nitrogen & we need to breath this to stay alive o_ \\
\hline doctor & hospital & person who one goes to see when one is sick d_ \\
\hline ocean & circumnavigation & n Pacific Indian and Atlantic are examples of o_ \\
\hline kitchen & stove & room in a building used to prepare food $\mathrm{k}_{\text {_ }}$ \\
\hline vaccine & immunity & this special form of injection is given to prevent disease $v_{-}$ \\
\hline christening & dedication & in what ceremony is an infant baptised $c_{-}$ \\
\hline freckle & birthmark & small brownish spots on the skin are called $f_{-}$ \\
\hline \multicolumn{3}{|l|}{ Block 4} \\
\hline horse & saddle & people like to ride and race these animals $\mathrm{h}$ \\
\hline tie & shirt & $\begin{array}{l}\text { object often worn around the neck and down the front by a man in } \\
\text { formal situations t_ }\end{array}$ \\
\hline umbrella & sunshade & one puts this up for protection in the rain $u_{-}$ \\
\hline tramp & hike & $\begin{array}{l}\text { in New Zealand people who put on a pack and boots and go into } \\
\text { the bush for a t. }\end{array}$ \\
\hline nails & carpenter & builders bang these into wood to hold it together $n_{-}$ \\
\hline King & emperor & in royalty the male sovereign is called a $\mathrm{k}$ \\
\hline hat & cap & object with a brim worn on the head $\mathrm{h}_{-}$ \\
\hline thunder & storm & very loud rumbling noise heard after lightening t_ \\
\hline ukulele & lute & a tiny guitar $u_{-}$ \\
\hline floor & carpet & one walks on this inside $f_{-}$ \\
\hline night & stars & the moon comes out at $n_{-}$ \\
\hline gold & copper & precious yellow covered metal g_ \\
\hline cigarette & ash & object which one smokes c_ \\
\hline dolphin & sea lion & an intelligent and playful sea creature $d_{-}$ \\
\hline plague & epidemic & what disease was called the black death $p_{-}$ \\
\hline & backgammon & board game which includes pawns and knights $c_{-}$ \\
\hline
\end{tabular}

